LCERN-PPE/94-94 Su:9428

Collège de France LPC $/ 9 ;-30$

15 June 1994

\title{
BEAM TESTS OF A FAST-RICH PROTOTYPE WITH VLSI READOUT ELECTRONICS
}

\author{
J. Séguinot*), T. Ypsilantis. and J.P. Jobez \\ Laboratoire do Physique Coippusculaire, Collège de France, F-75231 Parí Cedex 05, France. \\ R. Arnold and J.L. Guyonnet \\ CRN, Centre do Recherches Nucléaires, IN2P3-CNRS, Université Louis Pasteur, $r-67037$ \\ Strasbourg Cedex, France. \\ F. Chesi and J. Tischhauser \\ CERN, CH-1211 Genève 23, Switzerland. \\ I. Adachi and T. Sumiyoshi \\ KEK, National Laboratory for High Energy Physics, 1-1 Oho, Tsukuba-shi, Ibaraki-ken, 305, \\ Japan. \\ R.J. Mountain ${ }^{\dagger}$ \\ Department of Physics, Syracuse University, Syracuse, NY 13244-1130, USA.
}

\section{CERN LIBRARIES, GENEVA

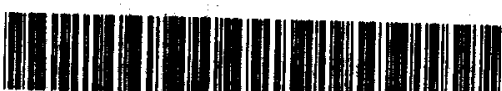 \\ CERN-PPE-94-94}

Fast-RICH Group

Abstract

In this report we discuss the Fast Ring Imaging Cherenkov tochnique that we have developed for application to proximity-focused $\mathrm{LiF}$ (or $\mathrm{CaF}_{2}$ ) solid radiator and multiwire chamber photon detector with cathode-pad readout using TEA in CI 4 as photosensor. We describe the full. scale Prototype of 12000 pads $\left(5.334 \times 6.604 \mathrm{~mm}^{2}\right)$ we have built, and briefly the dedirated VLSI readout elcctronics we have developed. We report in detail the investigations we have performed in a hadron test beam at the CERN PS, and compare the results obtained to the expected perfornances. The maximum momentum for $\pi / K$ separation at $3 \sigma$ achieved in these tests is $2.86 \mathrm{GeV} / \mathrm{c}$ for $\mathrm{LiF}\left(2.39 \mathrm{GeV} / \mathrm{c}\right.$ for $\left.\mathrm{CaF}_{2}\right)$. The expermentally achieved Cherentis merit factors, after correction for azinuthal angle aceptance, are $N_{0}=65.5 \mathrm{~cm}^{-1}\left(5.7 .7 \mathrm{~cm}^{-1}\right)$. to be compared with $53.8 \mathrm{~cm}^{-1}\left(50.2 \mathrm{~cm}^{-1}\right)$ from Monte Carlo calculations. Operation of the detector over several months has proven the technicue reliable and robust, and suitable for application in high-huminosity hadron colliders like LIIC, as well as $\epsilon^{+} e^{-} B$-Factories like bEK (Japan), SLAC (USA), and Comell (USA).

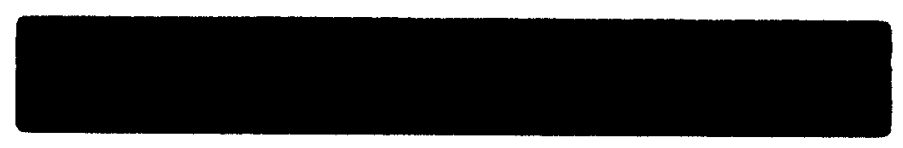

(To be published in Nuclear Instruments and Methods in Physics Research, Section, A)

\footnotetext{
* Corresponding author's mailing address: CERN. CH-1211 Genève 23, Switzerland.

ti Currently at: CERN / PPE Division, CH-1211 Genève 23, switzerland.
} 
We originally proposed in 1988 a Fast-RICH detector with a $3 \sigma \pi / K$ separation up to 4 (3) $\mathrm{GeV} / c$, using proximity-focused solid $\mathrm{LiF}\left(\mathrm{CaF}_{2}\right)$ crystal radiators and a fast electronic system to read out the cathode-pad array of a multiwire photon detector operated with TEA in $\mathrm{CH}_{4}$ for fast response. This project of $4 \times 10^{5}$ channels was designed for an $e^{+} e^{-}$collider $B$-Factory project at the Paul Scherrer Institute (P.S.I.) in Switzerland [1].

In 1988, the project was a challenge for the RICH technique which initiated detailed studies on detector response [2] and the development of two ASICs $[3,4,5,6]$, using the VLSI technique, for the readout. The basic concept was to fully integrate the electronics over the back side of the photon detector in order to drastically reduce the cabling with the counting room and to minimize both radiation length and dead space. Although the $B$-Factory project at PSI was abandoned, interest in the Fast-RICH technique for other projects ( $B$ physics at LHC at CERN in Europe, $B$-Factories at KEK in Japan, SLAC and Cornell in the USA) became strong, and we continued the development.

A full-scale Prototype of the barrel RICH detector, with 12000 channels, was built and the first results obtained with cosmic rays muons between December 1992 and March 1993 [7] proved that the technique worked well. Operation of the electronics over several months was very successful and the experimental results near to the expected performance. Nevertheless, small but significant discrepancies were observed in the number of photoelectrons $N_{\text {pe }}$ or in the single-photon Cherenkov angle resolution $\sigma_{\theta}$ for the $\mathrm{LiF}$ and $\mathrm{CaF}_{2}$ radiators (q.v. Table I) in these cosmic ray runs.

However because of the very low trigger rate $(\sim 12 /$ hour $)$, detailed investigations were difficult to perform. Moreover, the momentum acceptance of the muons above the imposed threshold $(500 \mathrm{MeV} / \mathrm{c}$ ) being not well-known, there was some uncertainty in the obtained Cherenkov angle resolution. Therefore, more precise measurements in a test beam environment were still very useful and even necessary.

This article discusses the results obtained during July 1993 in a test beam at the CERN PS. It is organized as follows. After a discussion in Section $\S 2$ of the physical and experimental choices we have made to conceive a Fast-RICH detector, we describe rather briefly in Section $\S 3$ the mechanical design of the Prototype and the fast VLSI readout electronics which was developed. Section $\S 4$ is devoted to the experimental test beam setup, and Section $\S 5$ to the physical properties of the components and the conditions of the Prototype operation. The data analysis procedure and calculations of the expected performance (both analytic and $\mathrm{MC}$ ) are given in Section $\S 6$. Finally the experimental results are detailed in Section $\S 7$, and are discussed with respect to the expected performance in Section $\S 8$.

\section{THE FAST-RICH TECHNIQUE}

A review of the Fast-RICH technique with pad readout may be found in $[8,9]$; however, we briefly comment in this section on the choices that we have made in the present detector development.

It is obvious since pad detectors are intrinsically and unambiguously two-dimensional, that they are well-suited to physics with high multiplicity events in a high-luminosity environment, provided these events can be selected in a short time interval (strobe-width) depending on the counting rate. For hadron colliders like LHC, with a $40 \mathrm{MHz}$ bunch-crossing rate, a strobe of less than $25 \mathrm{nsec}$ will be required to "disentangle" successive events. Moreover, if a trigger rejection level of $10^{3}$ is obtained by external logic, data readout at rates $\geq 100 \mathrm{KHz}$ will be needed for a data acquisition efficiency of $\geq 95 \%$. For an $e^{+} e^{-} B$-Factory, even if the interaction rate is small $(20-100 \mathrm{~Hz})$, large backgrounds of X-rays are expected at rates larger than $1 \mathrm{MHz}$, such that to obtain good image quality (optimal signal-to-noise ratio), hence optimal separating power, fast detector and front-end electronics are still required to suppress this noise. As the number of channels becomes very large for the detectors currently envisaged $\left(10^{5}\right.$ to $\left.10^{6}\right)$, this clearly imposes integrated electronics on the detector to readout the hit pads in situ with encoding and 
zero-suppression, using a minimum number of connections to the data acquisition system.

Obviously fast photon detectors require small time dispersion for photoelectron collection, and hence a short photon absorption length. At the time of our proposal in 1988, only photoionizing organic vapors of TEA or TMA admixed in $\mathrm{CH}_{4}$ could be used [12], TEA being preferred because it provides a larger merit factor $N_{0}$ and a $\sim 10$ nsec time dispersion at room temperature. Today, the trend is toward solid photocathodes of CsI, especially since our first measurements [12] showed high quantum efficiencies (20-30\%) are attainable in the reflective mode with isochronous electron extraction into $\mathrm{CH}_{4}$, as needed for amplification in multiwire detectors. This solid photocathode technique allows suppression of the photon detector windows, decreasing the total radiation length of the detector. In the near future the Prototype detector discussed in this paper will be tested with a CsI photocathode.

The photoelectron amplification element can be a MWC (multiwire chamber), a PPAC (parallel plate avalanche chamber), or a MSAC (multi-step avalanche chamber). However, only the first two techniques with a single amplifying gap can be used with a reflective photocathode. For a PPAC, the signal detected on the pads is induced by the avalanche development. It is rather slow since the total charge increases when drifting over the gap width. Moreover the gain attainable $\left(\sim 10^{4}\right)$ is so modest that the induced current is too small for a fast detection. Therefore, a Fast-RICH counter with a reflective photocathode can only be developed with a MWC. The positive induced signal on the pads by the drift of the positive ions towards the cathode has a fast rise time allowing the use of a fast current amplifier, if a gain of a few $10^{5}$ to $10^{6}$ can be reached.

A MSAC with anode pad can be used with photosensitive gas and is fast since avalanche electrons are collected on the pads. Their use is, however, only justified to eliminate the detection of photon feedback; however, there exists a major problem in maintaining a large electric field over a large surface which usually causes sparking and gain variation [13]. With TEA, the production of secondary electrons from atomic excitation levels is small in comparison with TMAE, and because of the short photon mean free path, it only contributes to the clustering size, improving in fact slightly the spatial resolution [2].

There are two strategies currently discussed for the pad readout of MWCs, namely, analog (measurement of induced charge on each pad), or digital (discriminated signal). An argument often given in favor of the former is better determination of the avalanche barycenter.

However, it must be pointed out that the resolution in the coordinate transverse to the wire direction is largely dominated by the wire spacing, since the photoelectron collection is quantized, and consequently not improvable by analog measurements, especially when the pad size is larger than the wire spacing. Moreover, as will be shown in Section $\S 6$ of this paper, for the often-used proximity-focused solid or liquid radiator, the dominant source of errors are the chromatic aberrations and a geometric error due to the radiator thickness. Hence an improvement of the pixel resolution in this case is not useful.

An improvement of the spatial resolution using analog measurements requires an anodewire to cathode-pad distance as large as possible with respect to the pad size in order to spread the induced charge on the pads around the avalanche center so as to interpolate for a good barycenter determination. This results in a large surface occupancy per photoelectron such that the probability of overlap of images for high multiplicity events becomes high. The pattern recognition is therefore difficult, worsening the identification power of the device.

Analog measurements require a charge amplifier with a long integration time as a consequence of the large anode-cathode distances imposed. Peaking times are at least $500 \mathrm{nsec}$. Thus the technique is not fast, and inadequate to separate events in a high-luminosity environment. Moreover, as ADCs are used for the readout with a multiplexing of channels, the readout also will be slow.

A last argument often advanced in favor of analog measurements is its ability to discriminate the track of a charged particle from the photoelectrons of the Cherenkov images, by using the large ratio of the corresponding signal amplitudes. The number of hit pads due the charged particle will consequently increase strongly, worsening the pattern recognition by overlapping 
the hit pads from different tracks in high multiplicity events. This argument in fact is surprising since the Cherenkov angle reconstruction requires accurate a priori knowledge of the particle direction. Therefore, the charged particle impact point is only useful for pattern recognition in association with a tracker coupled to the RICH detector, and perhaps also to improve the momentum determination by adding one more space point at a larger distance from the interaction point.

We have already shown [3], and the present tests confirm, that a digital readout of the hit pads can be fast, allowing a data sampling in 20 to $30 \mathrm{nsec}$, for anode-cathode distance as small as 0.4 to $0.5 \mathrm{~mm}$. With such an electrostatic configuration the coupling is strong, allowing a nearly full photoelectron detection efficiency by using a fast $(\Delta f \approx 50 \mathrm{MHz})$ low-noise current amplifier $\left(\sigma_{\text {e.n. }} \approx 10 \mathrm{nA}\right)$, when the avalanche gain is higher than $\sim 5 \times 10^{5}$. The multiplicity of hit pads per cluster is small $(\sim 1.25)$ for isolated photoelectrons, and since the detection is digital, the data acquisition can be fast.

A criticism often given of the present technique is the constraint of maintaining a small anode-cathode distance, a source of mechanical difficulties for construction and of sparking. We must stress that the wires are fixed at this distance every $\sim 10 \mathrm{~cm}$ along the wire by gluing to ceramic spacers. In a half year of operation, we have never broken a wire, even after generation of sparks. Moreover, concerning the reliability of the detector operation, it must be pointed out that the risk of sparking is related to the electric field. So, at constant gain, the problem is to first order independent of the anode-cathode distance. But of course, because of the small anode-cathode distance, the mechanical tolerance on the wire positioning is critical to guarantee a uniform gas gain over the surface of the detector, and for that reason the construction needs more care.

Finally, the technique assures a small ionization path length, which is a great advantage with regard to the production of secondary photons from $\mathrm{C}^{*}$ to get, as will be shown in this paper, good image quality.

As far as we know, there are several detector developments being pursued with analog readout $[13,14]$, but no equivalent Fast-RICH detector with digital readout.

\section{THE FAST-RICH PROTOTYPE}

As the Prototype and the VLSI readout electronics have already been discussed in previous articles, in which the details can be found, we give in this section an overall description of the detector and we summarize the main features needed for an overview of the technique and an understanding of the tests made.

\subsection{Mechanical Conception and Construction}

The mechanical structure of the Prototype shown in Figure 1 comprises three sectors, each of $12^{\circ}$ in azimuth between inner and outer radii of 65 and $85 \mathrm{~cm}$ respectively. ${ }^{1)}$ The length of $90 \mathrm{~cm}$ corresponds to the half-length of the proposed P.S.I. detector.

With three sectors, about $90 \%$ of the transmitted Cherenkov ring image is detectable if the beam is kept in the median plane defined by the central sector. Therefore, as drawn in Figure 1(b), only the central Sector 1 was equipped with radiators: three different $\mathrm{CaF}_{2}$ crystals $\left(140 \times 127 \mathrm{~mm}^{2}\right.$ each $)$, and two different LiF crystals $(\varnothing=100 \mathrm{~mm})$. They are each $10 \mathrm{~mm}$ thick, and are fixed on the honeycomb back structure of the Prototype at a distance of $13 \mathrm{~cm}$ from the windows, alternating in such a way that the $\mathrm{CaF}_{2}$ radiators are in positions $1,3,5$, and $\mathrm{LiF}$ in 2, 4, as numbered starting from one end (opposite the bus end) of the detector.

The $\mathrm{CaF}_{2}$ radiators are polycrystals manufactured by OPTOVAC ${ }^{2)}$ and distributed in Europe by $\mathrm{BDH}^{3}$, while the $\mathrm{LiF}$ radiators are monocrystals made by $\mathrm{BDH}$. Because of their

\footnotetext{
1) The three sectors are labelled Sector $0,1,2$ in this and forthcoming sections, as 0 is significant for the readout electronics.

2) OPTOVAC Co., East Brookfield Road, North Brookfield, MA 01535, USA.

3) BDH Ltd., Broom Road, Poole BH12 4NN, England.
} 
circular shape the LiF crystals are centered in a plexiglas frame $\left(140 \times 127 \mathrm{~mm}^{2}\right)$ and mounted alongside the $\mathrm{CaF}_{2}$ radiators. All crystals are optically polished on both parallel faces only. Therefore some fraction of the UV Cherenkov light, trapped inside the crystal by total internal reflection, could escape the radiator by isotropic diffusion on the sides, and thus contribute to the image background. To study this effect, silicon vacuum grease was deposited onto the lateral circumference of radiators \#4 ( $\mathrm{LiF})$ and \#5 $\left(\mathrm{CaF}_{2}\right)$ to absorb these UV photons. No such effect was subsequently found.

The drawings in Figures 1(a) and 1(b) show the $\mathrm{CaF}_{2}$ windows $\left(170 \times 162 \mathrm{~mm}^{2}\right.$ each, $3.5 \mathrm{~mm}$ thick) glued on a rigid aluminum frame, of $91 \%$ optical transparency at normal incidence. The parallelepipedic hollow structures, visible above the windows of each sector, receive the MWC photodetectors such that the cathode-pad plane forms with the windows a photon conversion gap of $3.5 \pm 0.1 \mathrm{~mm}$. This gap is thick enough to assure a full conversion efficiency ( $\left.>5 \ell_{\text {abs }}\right)$, with only a small ionization signal from charged particles $\left(21 e^{-}\right.$total at normal incidence ). The grooves distributed along the sides of the structure allow a transverse flow into each sector of the $\mathrm{CH}_{4}+\mathrm{TEA}\left(17^{\circ} \mathrm{C}\right)$ gas mixture, assuring an homogeneous and uniform gas filling. To define the multiwire cathode entrance plane, silver traces $100 \mu \mathrm{m}$ wide have been vacuum-deposited on the inner surface of the $\mathrm{CaF}_{2}$ windows, with a pitch of $1.5 \mathrm{~mm}$ transverse to the detector axis and a transparency of $93 \%$ [15].

The cathode-pad array is comprised of 30 columns (5.334 mm pitch) of 128 pads ( $\Delta x \times$ $\Delta z=5.334 \times 6.604 \mathrm{~mm}^{2}$ ) each, hence 3840 electronic readout channels per sector. The pad size was chosen to obtain an rms spatial resolution of $\sim 1 \mathrm{~mm}$ in each direction [2]. The gold-plated tungsten anode wires $(15 \mu \mathrm{m} \varnothing)$ are strung along the sector axis with a pitch of $1.27 \mathrm{~mm}$ at a distance $d=0.5 \mathrm{~mm}$ from the pad plane so as to maximize the induced pad signal [2] for a fast and efficient detector response. The wires are glued to $0.5 \mathrm{~mm}$ thick insulating ceramic spacers ( $2.2 \mathrm{~mm}$ wide, $10 \mu \mathrm{m}$ thickness tolerance) fixed on the cathode plane at $10.6 \mathrm{~cm}$ intervals (16 pad lengths, assuring a $94 \%$ surface detection efficiency) to guarantee good accuracy on the wire position, hence uniform gas gain and mechanical stability since electrostatic deflection is minimized.

As the pads are grounded through the electronic front end, the electrostatic configuration of the MWCs is defined by applying a negative potential $U_{W}$ to the strips on the windows (via the metallic frame) and a positive potential $U_{a}$ to the anode wires.

The cathode-pad array of each sector is supported by a rigid aluminum tray-like structure, $30 \mathrm{~mm}$ deep, housing the electronic readout (Figure 2), which fits into the hollows in the main structure of Figure 1(a). The tightness is obtained by a viton O-ring. The parallelism between the radiators and the windows is thus kept within $0.2 \mathrm{~mm}$ over the full length and the accuracy on the relative angle of two adjacent sectors within 2 mrad. In operation, this design allows detector replacement without disturbing the gas in the lever arm region and provides a very good mechanical rigidity. More details on the design and the fabrication of the cathode-plane seen in Figure 3 can be found in refs. [3, 16].

The cathode-pad plane is a composite of two 0.8 and $2.4 \mathrm{~mm}$ thick vetronite sheets (glass fiber and epoxy resin), sandwiching a thin $35 \mu \mathrm{m}$ copper foil. The perimeter of the copper foil is connected to the grounded support tray with silver glue to provide electrostatic shielding of the pad array as well as a good ground for the sensitive fast analog preamplifiers. The support tray is made of Aluminum and is specially treated with Alodine 1200 for good surface conductivity. The connection between a pad and its preamplifier is effected through rivets in metallized holes which pass through the grounded $\mathrm{Cu}$ foil.

Pads are made by etching, in the last phase of the fabrication, after gluing a $35 \mu \mathrm{m}$ thick copper foil locally connected to the metallized hole with conductive glue. The pads are finally plated with few $\mu \mathrm{m}$ thickness gold.

The inner surface of the radiator support is covered with a grounded $50 \mu \mathrm{m}$ thick Aluminium foil to minimize the outgassing of the structure and also to shield the front end electronics. 


\subsection{The Readout Electronics}

Each sector of 3840 cathode-pads ( 30 columns of 128 pads each) are read out in parallel by means of the two ASICs that we have developed in collaboration with the Micro-electronics Group of Rutherford Laboratory ${ }^{4)}$. These chips are:

- an analog 8-channel fast bipolar current preamplifier-amplifier and discriminator chip (RAL110) with current output; and

- a digital 16-channel CMOS chip (RAL111) that assures readout at high speeds of $\leq 20 \mathrm{MHz}$ with zero suppression and address encoding.

Detailed descriptions and studies can be found in refs. $[3,4,5,6]$.

Each digital CMOS chip forms a chip-set with two analog bipolar chips (see Figure 4), mounted on opposite sides of eight-layer printed-circuit boards (PCBs, see Figures 2 and 3), which transmit power, control and data lines. Interchangeable cards of 64 channels (i.e., four digital chips) are plugged vertically into the back side of the cathode-pad plate in 15 columns per sector, such that the board's pins connect through to the pad rivets. Each column thus contains four daisy-chained cards comprising 256 channels which are symmetrically connected to two adjacent columns of 128 pads each. The 15 columns form an array of 240 digital chips (3840 channels). They are connected to a common output top bus via drivers.

Individual cathode-pads are connected to the input of the analog chip with a $270 \Omega$ serial resistor (Figure 3) for protection against detector sparking; this protection is completed by two parallel oppositely-biased fast diodes (the cathode of one diode is grounded and the anode of the other diode is coupled to $-3 \mathrm{~V}$, due to the input transistor polarization).

\subsubsection{The Analog Chain} elements:

The 8-channel analog chip (RAL110) in bipolar technology is composed of four basic

(i) a fast current input stage (with an input impedance of $\sim 100 \Omega$ and an input RC timeconstant of $\sim 10 \mathrm{nsec}$ );

(ii) an amplifier stage;

(iii) a level shifter; and

(iv) a discriminator.

The design is fully symmetric, with inputs to the emitter of a common base transistor configuration, to enhance the power supply common mode rejection. The bandwidth of the amplifier stage is limited to $50 \mathrm{MHz}$ ( $7 \mathrm{nsec}$ rise-time at the output). The DC noise equivalent current (NEC) at the input is $\sigma_{\text {e.n. }} \approx 10 \mathrm{nA}$, which for an RC response-time of $10 \mathrm{nsec}$ corresponds to an equivalent input noise charge of $625 \mathrm{e}$. However, as shown in ref. [3], the noise is roughly doubled with the chips mounted on the PCBs and installed on the detector (so that $\sigma_{\text {e.n. }} \approx 20$ $\mathrm{nA}$ or $\sim 1250 \mathrm{e}$ ). The discriminator provides output in "current mode" in order to reduce voltage feedback to the inputs. The discriminator threshold, common to the eight channels of the analog chip, is set via an input current by a 4-bit DAC addressable from the console of the data acquisition system using the data bus. The correspondence between the analog mean input current and the DAC value (see Figure 24 in [3]) is $\bar{I}_{\text {in }}[\mathrm{nA}] \approx 20 \mathrm{DAC}$. Therefore, one DAC unit corresponds to about one rms unit $\sigma_{\text {e.n. }}$ of the current input noise in operation.

The cross-talk between channels is better than $40 \mathrm{~dB}$ and the power consumption is $\sim 10$ $\mathrm{mW}$ per channel. An analog fast OR of the eight channels is implemented and two differential test inputs can be used to inject test currents into the preamplifier inputs. Obviously due to the symmetric design the chip can either accept positive or negative input currents depending on the bonding scheme at the packaging stage.

\subsubsection{The Digital Readout Chip}

The digital CMOS chip (RAL111) was designed for a fast readout of sparse data (hit pads) of a selected event latched only during the time duration of a strobe signal to reject background

\footnotetext{
4) Micro-electronics Group, Rutherford-Appleton Laboratory, Chilton, Didcot, Oxon OX11 OQX, UK.
} 
(i.e., non-coincident events, electronic and detector noise). The strobe width $\Delta t$ is adjusted to accept the full spread of photoelectron arrival times, which for TEA is $\Delta t \geq 3 \sigma \approx 30 \mathrm{nsec}$. To compensate the delay between the discriminator current outputs of the analog chip and the strobe generated by an external trigger, each channel of the digital chip contains a 64-cell 50 $\mathrm{MHz}$ shift register to pipeline the input data during $1.32 \mu \mathrm{sec}$.

The input data are loaded by means of a synchronizer only once irrespective of the pulse duration, a fundamental feature of this chip necessary for good image quality. When an end pulse from the shift register is strobed, a datum is latched into the input memory of a priority encoder (q.v. Figure 5). The $50 \mathrm{MHz}$ clock of the shift registers is supplied by a common oscillator to each 64-channel card.

Once the readout process is initiated, a readout clock of maximum frequency $20 \mathrm{MHz}$ is generated and synchronously distributed to the chip array of each sector. Addresses of detected hits in the 16 channels of a chip are encoded by the 4-bit priority encoder and loaded into a 12-bit RAM-based FIFO memory, 16 words deep and located in the chip. Data transfer from the first chip column is then enabled while, in parallel, data compression is effected in the 14 remaining columns. When the first column is emptied, requiring at least 16 clock cycles, data transfer from the second column is started-but because of the parallel data compression, this requires only one clock cycle per hit. This process continues until the last chip column is emptied. Addresses of the row position ( 5 bits) are encoded by counters and associated to the channel addresses (4 bits). Column addresses are encoded by the data acquisition system ( $c f . \S 3.3$ ).

Total readout time is mostly defined by the number of image points (noise is very small: $\lesssim 1 \mathrm{hit} /$ sector per event), i.e., for $20 \mathrm{MHz}$ readout clock, it is $50 \mathrm{nsec}$ per hit plus $1.55 \mu \mathrm{sec}$ for initialization.

The digital chip contains the 4-bit DAC register, loadable via the four data bus lines, which provides the digital code to four external registers to supply the threshold current to the associated pair of analog chips. The digital chip also contains various facilities to check the correct operation of the readout chain by loading programmable hit patterns, via the data bus, at different levels of the chip structure.

The power consumption of the digital chip is about $6-8 \mathrm{~mW} /$ channel, hence with the ana$\log$ chip, a total of $\sim 17 \mathrm{~mW} /$ channel or $65 \mathrm{~W} /$ sector. To keep the temperature of the electronics at about $30^{\circ} \mathrm{C}$, sectors were cooled by forced-air circulation.

\subsubsection{Electronics Settings for Optimal Detector Operation}

For reasons of convenience, only 14 columns of readout electronics out of a total of 15 columns per sector were instrumented, hence $3 \times(14 \times 16)=672$ digital chips in toto $(10472$ channels).

During operation in the test beam, the strobe width was $50 \mathrm{nsec}$ and the discriminators set at a threshold equivalent to $\mathrm{DAC}=6$ (or $6 \times \sigma_{\text {e.n. }} \approx 120 \mathrm{nA}$ ) for most chips, except for 9 , 7 , and 4 noisier chips in Sectors 0,1 , and 2 respectively set at $\mathrm{DAC}=7$. This represents the standard DAC configuration.

For such a standard DAC setting, the mean number of raw hit pads per trigger due to the electronic noise was less than $0.5,0.2$, and $1.0 \mathrm{hits} /$ trigger for Sectors 0,1 , and 2 respectively. With a DAC setting of 7 applied to all chips, these numbers fall to $0.07,0.01$, and 0.2 hits $/$ trigger. A DAC $=7$ setting was chosen for those digital chips of which one or more of the 16 channels exhibited a counting probability larger than $1 \%$ at $\mathrm{DAC}=6$.

We must point out that during the cosmic ray tests (between December 1992 to March $1993, q . v .[7])$, the input of the analog chain was not protected from large negative current pulses due to sparking. When we designed the $\mathrm{PCB}$, it was thought that, because of the asymmetric multiwire structure, only positive currents could be drained by the cathode-pads, since a positive potential is applied to the anode wires. It was checked in test bench studies that this protection was efficient, solving the problem of available space on the PCBs to implant more components. Unfortunately, during detector operation it appeared that sometimes a micro-discharge between anode wires and cathode-pads can propagate, with delay, in the opposite gap, up to 
the negatively-polarized window. In such a manner, a negative current is directly drained to/in the lowest impedance cathode-pads.

Because of this mechanism, we lost some electronic channels during the cosmic ray tests. The problem was easily overcome by adding, on a complementary narrow PCB, as explained above in $\S 3.2$, a second diode with its anode at $-3 \mathrm{~V}$ as determined by the emitter polarization of the input transistor. Therefore, for the beam tests, there was about $0.1 \%, 1.5 \%$, and $5.5 \%$ dead channels in Sectors 0, 1, and 2. This protection proved to be quite adequate, and after the correction, no more channels were destroyed by this mechanism.

\subsection{The Data Acquisition System}

The layout of the data acquisition system is shown in Figure 6. The three sectors of the Prototype and the four MWPCs (discussed below) being read out in parallel, a CAMAC interface and VME memory are needed per readout channel (i.e., sector or MWPC) although, for clarity, only one is represented in the diagram. The present system works in single-event mode, but should be improved in the future to run in multi-event mode.

The real-time data acquisition system uses the SPIDER program provided by the CERN ECP/DS group, running under the OS-9 environment [17]. The DAQ control program drives the VLSI readout electronics via the CAMAC interfaces. Each CAMAC interface receives, from the 12-bit data bus of the associated sector (or MWPC), an encoded word per hit pad (or hit wire) containing the row address of the digital chip ( 5 bits) and the channel address ( 4 bits) in the chip. A specific combination of 2 of the last 3 bits is recognized by the interface as the readoutend flag of a column. Counting these flags (in 4 bits) defines the column address appended to the aforementioned row and channel addresses. The word address thus formed is transmitted to the VME dual port memory synchronously with the readout clock of the VLSI electronics. A clock readout is generated in each CAMAC interface during the data readout time interval only. The last bit of the data bus provides a BUSY flag during the readout.

A LAM (wired OR of all BUSY lines), generated when the most populated sector is read out, is the flag that starts the readout of the VME memories by the microprocessor. At this stage, the sector address and the event number are appended to form an unambiguous 32-bit word address of a hit pad.

A partial on-line analysis is performed during data-taking for monitoring purposes. At the end of a run, the data are automatically transferred via ethernet to the laboratory VAX, and stored on disk for off-line analysis.

Besides the DAQ control program, other user-written programs have been developed to drive the digital chips via the CAMAC interface and the 8-bit control lines by addressing each chip $(15 \times 16$ per Prototype sector, $2 \times 8$ per MWPC). The purpose being:

(i) to set the 16-channel discriminator threshold of the two associated analog chips; and

(ii) to perform diagnostics on the readout chain.

In the former case, the application is straightforward. In the latter case, random patterns are generated and stored in the register of the digital chips, a readout process is then started, and a comparison is made between the generated patterns and readout data. A diagnostic of the possible errors is also given by these test programs. The readout process is either slow (using only the CAMAC readout step-by-step), or fast (by directly reading the VME memories).

It should be noted that, because of the present interface design working in single-event mode, the data acquisition rate was limited to 60 events per burst of particles of $300 \mathrm{msec}$ duration, far from the ultimate performance of the readout system.

\section{EXPERIMENTAL TEST BEAM SETUP}

\subsection{Test Beam and Layout}

Tests of the Prototype have been performed in the T9 test beam at the CERN PS in July 1993 , with a typical intensity of $8 \times 10^{4}$ pions per second at $(10 . \pm 0.1) \mathrm{GeV} / c$. The beam could 
also be tuned to momenta as low as $2 \mathrm{GeV} / c$ in both polarities. Because of the very long distance between the target and the detector, the kaon and muon content of the beam is negligible.

The test setup shown in Figure 7(a) was designed to accurately reconstruct the beam particle trajectory with four MWPCs, (three upstream of the Prototype), in order to determine the Cherenkov angle of the detected photoelectrons with negligible experimental error on the incident track.

\subsection{Scintillation Counter Telescope}

The beam particles were detected by a telescope of six scintillation counters ( $S_{1}$ to $\left.S_{6}\right)$ in a crossed configuration, yielding a roughly Gaussian acceptance in $x, z$ (the transverse plane) with standard deviations $\sigma_{x} \approx \sigma_{z} \approx 2 \mathrm{~cm}$. The halo counter $S_{3}$, of $30 \times 30 \mathrm{~cm}^{2}$ active area with a hole of $4 \mathrm{~cm}$ diameter centered on the beam axis, rejected multiparticle events resulting from interactions in upstream collimators.

\subsection{MWPCs and Alignment}

Four MWPCs (MWPC0 to MWPC3) were mounted on a test bench built to allow precise alignment, with the relative distances as well as the counter geometry indicated in Figure 7(b). The MWPCs each have two orthogonal planes of 96 wires ( $\varnothing 16 \mu \mathrm{m}$, gold-plated tungsten) with $1.27 \mathrm{~mm}$ spacing and $4.8 \mathrm{~mm}$ between the cathode planes $(20 \mu \mathrm{m}$ aluminized mylar foils $)$. The MWPCs were flushed with a $90 \% \mathrm{CH}_{4}+10 \%\left(\mathrm{iC}_{4} \mathrm{H}_{10}+\right.$ isopropyl alcohol $)$ gas mixture, and read out with the same VLSI electronics as used for the Prototype, but employing the negative input polarity of the analog chip.

The initial chamber alignment was performed with an accuracy of $0.1 \mathrm{~mm}$ by means of three survey sights per chamber mounted on cross-bars above the chambers (visible in Figure $7(\mathrm{a})$ ): sights at the extremities were prealigned at a known distance with respect to the horizontal wires, whereas a central sight marks the middle of the vertical wire plane. For more information, refer to [7].

The Prototype was placed between MWPC2 and MWPC3 and mounted in a frame fixed on a turntable with a vertical axis of rotation exactly centered on the beam line (Figure 7(a)). The Prototype coordinate system was defined with $z$ along the wires (horizontal direction), $x$ across the wires (vertical direction), and $y$ normal to the radiator plane. The Prototype could also be rotated around a horizontal axis to allow an accurate adjustment of the beam angle of incidence $\left(\theta_{p}, \phi_{p}\right.$ polar and azimuthal angles) with respect to the normal of the radiators; moreover the frame supporting the Prototype could be translated on an I-beam rail structure in order to select one of the five radiators.

The relative position of the MWPCs were finally slightly corrected by software such that the distribution of residuals between the measured (surveyed) coordinates and the corresponding position calculated from a least squares straight line fit to the wire hits is centered at zero. The rms deviations of these distributions after such optimization are of the order of $220 \mu \mathrm{m}$ (with $1.27 / \sqrt{12}=367 \mu \mathrm{m}$ ) for MWPC0 to MWPC2.

\subsection{Beam Track Reconstruction}

A particle trajectory was reconstructed using only those planes in which there was a single wire-cluster detected. Single-cluster planes were required in both views for the first and last chamber (MWPC0 and MWPC3), whereas only one such plane was required between the middle chambers (MWPC1 and MWPC2). Hence three out of four possible coordinates were required. An error $\sigma_{x z}=1.27 / \sqrt{12} \mathrm{~mm}$ was attributed to the position of each cluster in MWPC0 to MWPC2, and an error $\sigma=\sqrt{\sigma_{x z}^{2}+\sigma_{\mathrm{ms}}^{2}}$ to that in MWPC3. Here $\sigma_{\mathrm{ms}}$ is the contribution due to multiple scattering calculated for each incident angle. A least squares fit was made for each view, and the event kept as a "good event" if the probability of the track hypothesis gave $P\left(\chi^{2}\right)$ greater than $1 \%$.

Due to the location of MWPC3 downstream of the Prototype, this procedure selects events with single stiff tracks, reducing the contribution of multiple scattering in the Prototype 
and allowing clean Cherenkov ring reconstruction. Therefore, taking into account all of these considerations, the fraction of good events with respect to the trigger rate was about $66 \%$.

The calculation of the position of the Prototype relative to the MWPCs was determined with an accuracy of $<0.2 \mathrm{~mm}$ by measuring the barycenter of the beam impact in the test runs without TEA; the detector being positioned normal to the beam line as drawn in Figure 7(a).

\section{PROTOTYPE OPERATION AND PHYSICAL PROPERTIES OF COMPONENTS}

\subsection{Photon Mean Free Path and Quantum Efficiency of the Photodetector Gas Mixture}

During operation the photodetectors were flushed with $10 \ell / \mathrm{h}$ of $\mathrm{CH}_{4}(\mathrm{~N} 35)$, cleaned by an oxisorb cartridge, and bubbled through TEA kept in a bath at $T_{b}=290 \mathrm{~K}$. The carrier $\mathrm{CH}_{4}$ gas was thus saturated with TEA at a partial vapor pressure $P_{\text {vap }}$ given by the Clausius-Clapeyron equation, viz.,

$$
P_{\text {vap }}=P_{0} \exp \left[\left(\frac{\Delta H}{R}\right)\left(\frac{1}{T_{0}}-\frac{1}{T_{b}}\right)\right]
$$

where $P_{0}$ and $T_{0}$ are the reference pressure and temperature, and $(\Delta H / R)$ is the ratio of the latent heat of vaporization and the ideal gas constant, which is the slope of the equation. For TEA, $P_{0}=73.2$ torr at $T_{0}=300 \mathrm{~K}$, with $(\Delta H / R)=4299 \mathrm{~K}[18]$, giving $P_{\text {vap }}=44.7$ torr, at bubbler temperature $T_{b}=290 \mathrm{~K}$.

The photoabsorption length $\ell_{\mathrm{abs}}$ of TEA $\left(\right.$ or $\left.\mathrm{CH}_{4}\right)$ is

$$
\ell_{\mathrm{abs}}=\frac{k_{B} T_{\mathrm{det}}}{P_{\mathrm{vap}} \sigma_{\mathrm{abs}}}
$$

where $T_{\text {det }}$ is the detector temperature, stabilized during operation to $\sim 303 \mathrm{~K}$ because of the electronic heat dissipation, $P_{\text {vap }}$ is the partial vapor pressure, and $\sigma_{\mathrm{abs}}$ is the photoabsorption cross section of TEA (or $\mathrm{CH}_{4}$ ). Boltzmann's constant is $k_{B}=1.036 \mathrm{~mm} \cdot$ torr $\cdot \mathrm{Mb} / \mathrm{K}$.

The photoabsorption cross sections of TEA and $\mathrm{CH}_{4}$ are shown in Figure 8(a). Photoabsorption in TEA is known from ref. [19], but for $\mathrm{CH}_{4}$ a measurement was performed with a monochromator and a gas test cell $23.6 \mathrm{~cm}$ long at the output of the photodetectors during the period of rinsing in order to take into account the residual impurities from outgassing. The absorption length in $\mathrm{CH}_{4}$ is dominant below $133 \mathrm{~nm}$, i.e., above a photon energy of $8.9 \mathrm{eV}$.

The absorption length $\ell_{\mathrm{abs}}$ for the $\mathrm{CH}_{4}+\mathrm{TEA}$ gas mixture, viz.,

$$
\ell_{\mathrm{abs}}=\frac{\ell_{\mathrm{CH}_{4}} \cdot \ell_{\mathrm{TEA}}}{\ell_{\mathrm{CH}_{4}}+\ell_{\mathrm{TEA}}}
$$

calculated as the reciprocal sum of the absorption lengths of the two component gases for test operating conditions, is shown in Figure 8(b) as a function of wavelength. This curve was used for the simulation program discussed below. At the maximum of the TEA quantum efficiency $(8.3 \mathrm{eV} \approx 150 \mathrm{~nm}) \ell_{\mathrm{abs}}=0.6 \mathrm{~mm}$; for full absorption $\left(4-5 \ell_{\mathrm{abs}}\right)$ and normal incidence to the photodetectors, the rms deviation for photoelectron collection is less than $10 \mathrm{nsec}$, hence very fast.

The TEA quantum efficiency measurements in Ar or He as carrier-gas of $[2,10]$ are in good agreement. In Figure 9 we show measurements $[2,19]$ in Ar and the calculated QE,

$$
Q_{\mathrm{mix}}=Q_{\mathrm{TEA}}\left[1+\frac{\left(P_{\mathrm{vap}} \sigma_{\mathrm{abs}}\right)_{\mathrm{CH}_{4}}}{\left(P_{\mathrm{vap}} \sigma_{\mathrm{abs}}\right)_{\mathrm{TEA}}}\right]^{-1},
$$

weighted by the relative photoabsorption in TEA vapor with respect to the $\mathrm{CH}_{4}+\mathrm{TEA}$ gas mixture used under beam test conditions. 


\subsection{Refractive Index of the $\mathrm{CaF}_{2}$ and $\mathrm{LiF}$ Radiators}

To determine the refractive indices of the radiators in the UV region with a reasonable degree of confidence, we have used the data compilation by H.H. Li for $\mathrm{CaF}_{2}$ [21] and $\mathrm{LiF}$ [20]. In the former case, we have also included data from the compilation of Landolt-Börnstein [22] as well as the most recent measurements of M. Hempstead et al. [24] below $8.3 \mathrm{eV}$. In the latter case, we have added the data of Laporte et al. [23]. These data are shown in Figure 10 along with our 2-pole Sellmeier fits determined from these data and used in the simulation program. The $N$-pole Sellmeier formula in the energy metric is of the form

$$
F(E)=\sum_{i=1}^{N} \frac{F_{i}}{\left(E_{i}^{2}-E^{2}\right)}
$$

where $F(E)$ is proportional to the molar refractivity and is here given either by the LorentzLorenz form $\left[\left(n^{2}-1\right) /\left(n^{2}+2\right)\right]$ or by $\left(n^{2}-1\right)$. The form of $F(E)$, the resonance energies $E_{i}$, and the coefficients $F_{i}$ proportional to the oscillator strengths are listed in Table II.

In the interval of energy acceptance of TEA in $\mathrm{CH}_{4}(7.5<E<9.3 \mathrm{eV})$, the data-sets for $\mathrm{LiF}$ are in fair agreement with each other. To the contrary, the dispersion of the data points is large for $\mathrm{CaF}_{2}$. The difference in chromaticity between both radiators is clearly evident.

For the analysis of the cosmic ray data [7], we used the other fits shown in Figure 10. Specifically, in the analysis of the $\mathrm{CaF}_{2}$ cosmic ray data, we used the fit labelled "Malitson" in Figure 10(a), which agreed with the recent measurements of Hempstead et al. [24]. In the LiF cosmic ray data analysis, the fit used (and shown in Figure 10(b)) was the one recommended by $\mathrm{Li}[20]$-surprisingly, as we discovered later, obtained by extrapolation of low photon energy data. However, the smallish discrepancies observed in the single-photon angular resolution for both radiators suggested an underestimated contribution from chromatic error, hence the present fit.

\subsection{UV Transmission of Radiators and Windows}

The UV transmission measurements at normal incidence of a $10 \mathrm{~mm}$ thick LiF radiator, and a $5 \mathrm{~mm} \mathrm{CaF}_{2}$ sample of the radiator quality crystal (i.e., from the same production run) are shown in Figure 11(a), along with the measurements for the $3.5 \mathrm{~mm}$ thick $\mathrm{CaF}_{2}$ window [15].

Nevertheless, for the MC calculations we need the absorption coefficients ${ }^{5)} \mu$ to attenuate the Cherenkov photon flux transported across the bulk of the various media, since reflectivity of the polarized Cherenkov light at each interface is a function of the incident angle.

The measured (external) transmittances $T=T(E)$ at normal incidence were used to calculate for each photon energy $E$ the bulk (internal) transmittances $t=\exp (-\mu \ell)$ from

$$
T=\frac{(1-R)^{2} t}{1+R^{2} t^{2}}
$$

where $\mu$ is the absorption coefficient, $\ell$ is the sample thickness, $R=[(n-1) /(n+1)]^{2}$ is the reflectivity at each interface, and $n$ is the refractive index determined by the Sellmeier fits discussed above. This formula was derived in the case of normal incidence and small material absorption. Given a measured $T$ and calculated $n$, solving this equation for $t$ allows the determination of the attenuation coefficient as $\mu=-\ln (t) / \ell$ at each photon energy $E$ (q.v. Figure 11(b)).

\subsection{UV Transmission of Lever Arm Gas Volume}

In operation, the lever arm volume between the radiators and the photodetector windows was flushed with $50 \mathrm{\ell} / \mathrm{h}$ of Argon-hence a renewal of the volume every hour-passed through two oxisorb cartridges in series for cleaning. This was the condition, as shown in Figure 12, to

5) One must in general make a formal distinction between attenuation and absorption, as the former mechanism consists of both true atomic or molecular absorption in a medium and scattering effects as well. However, for media of present interest, scattering effects are negligible, so our usage will conform to the more conventional mix of these two terms. The distinction is more problematic in the case of, e.g., silica aerogel. 
get a UV transparency of about $95 \%$ in the TEA energy acceptance interval $(7.5<E<9.3 \mathrm{eV})$ for a $23.6 \mathrm{~cm}$ path length, as monitored at the detector output with a monochromator.

The threshold of the absorption, observed at $184 \mathrm{~nm}$, is near to the onset of a sizeable photoabsorption cross section of water $\left(186 \mathrm{~nm}\right.$, with $\sigma_{\mathrm{abs}}\left(\mathrm{H}_{2} \mathrm{O}\right)=5 \mathrm{Mb}$ at $165 \mathrm{~nm}$ and $0.2 \mathrm{Mb}$ at $146 \mathrm{~nm})$, and that of oxygen $\left(177 \mathrm{~nm}\right.$, with a maximum $\sigma_{\text {abs }}\left(\mathrm{O}_{2}\right) \approx 12 \mathrm{Mb}$ in the interval $138-146 \mathrm{~nm})$.

Therefore, the measured transmission indicates that with an Argon flow-rate of $50 \mathrm{l} / \mathrm{h}$, there is a residual contamination of about $7 \mathrm{ppm}$ of $\mathrm{O}_{2}$ from an air leak, or maybe less if, as is probable, it is combined with an outgassing of adsorbed water vapor from the Prototype structure itself.

\section{DATA ANALYSIS AND EXPECTED PERFORMANCE}

\subsection{Analytical Calculation of the Resolution}

We here use the analytical formulation $[9,11]$ for the optics of proximity-focused radiators. The various contributions to the single-photon Cherenkov angle resolution $\sigma_{\theta}$ as a function of the azimuthal Cherenkov angle $\phi$ have been calculated and are shown in Figure 13 for our two standard radiators: $\mathrm{CaF}_{2}$ at $\theta_{p}=30^{\circ}$ and $\mathrm{LiF}$ at $\theta_{p}=25^{\circ}$. Shown also is the total photon transmission through the bulk media including reflection losses at the interfaces. The calculation uses piece-wise integration of the TEA quantum response (Figure 9), with a constant absorption length $\ell_{\mathrm{abs}}=0.6 \mathrm{~mm}$ (Figure $8(\mathrm{~b})$ ), and assumes all errors are Gaussian. The bulk attenuation coefficients used are the measured values in each medium at the average photon energy of 8.36 $\mathrm{eV}$ (Figures 11(b) and 12).

The total resolution $\sigma_{\theta}$ is clearly dominated by the chromatic error $\sigma_{\theta}(E)$. The pixel error $\sigma_{\theta}(x, y)$ and radiator thickness error $\sigma_{\theta}\left(z_{e}\right)$ are negligible for $10^{\circ} \leq \theta_{p} \leq 30^{\circ}$, but the latter becomes important at the larger incident angles $\left(40^{\circ} \leq \theta_{p} \leq 60^{\circ}\right)$ needed for barrel detectors in collider geometry. However, increasing the pad size is not a good strategy because it decreases the two photon separation probability and results in a two-way loss in the event angular resolution $\sigma_{\theta}$ evt $=\sigma_{\theta} / \sqrt{N_{\text {pe }}}$ (i.e., $N_{\text {pe }}$ is reduced and $\sigma_{\theta}$ is increased as discussed in $\S 8$ ).

The analytical calculation gives the single-photon Cherenkov angle resolutions: $\sigma_{\theta}=19.6$ mrad for $\mathrm{CaF}_{2}$ and $14.6 \mathrm{mrad}$ for $\mathrm{LiF}$, by averaging over azimuthal angles the total error of Figure 13 weighted by the transmission using our fits to the refractive indices (see Table II). With the (Malitson, Li) fits, the corresponding total errors are $\sigma_{\theta}=(18.2,12.0)$ mrad. Moreover, as is shown experimentally and by Monte Carlo simulation, because of tails in the quantum response function coupled to a strongly rising refractive index function, the Cherenkov angle distribution becomes somewhat non-Gaussian causing a difference between the analytic and the MC predictions for the widths.

The mean Cherenkov angles from the analytical calculation are $\bar{\theta}=887.9$ and 843.8 mrad and the per event photoelectron yields $N_{\text {pe }}=9.86$ for $\mathrm{CaF}_{2}$ and 10.81 for LiF radiators, respectively, for $10 \mathrm{GeV} / c$ pions in the actual detector geometry with the additional transmission factors $T=T_{\mathrm{da}} \cdot T_{\mathrm{tr}}=(0.904)(0.93)=0.841$ described in $\S 6.4$.

Following the approach of [8] for calculation of the Cherenkov merit factor $N_{0}$, the energy integrated quantum response of this photosensor is $\langle Q\rangle \Delta E=0.30 \mathrm{eV}$, hence

$$
N_{0}=(370 / \mathrm{eV} \cdot \mathrm{cm}) T^{2}\langle Q\rangle \Delta E=62.4 \mathrm{~cm}^{-1}
$$

is predicted for both $\mathrm{LiF}$ and $\mathrm{CaF}_{2}$ radiators, using the default value $T=0.75$ for both radiator and window transmissions.

\subsection{Monte Carlo Simulation}

The Monte Carlo simulation and reconstruction programs have been developed and tested for various RICH detectors over the past dozen years in order to interpret experimental results and reduce raw data. The MC program simulates all the processes from photon emission to 
photoelectron detection, taking into account the detector geometry (with the photodetector support structure, the window frames, and the ceramic spacers of the MWCs), the physical properties of the optical media ( $c f . \S 5)$, along with the multiwire and associated electronic responses studied in ref. [2]. Nevertheless, for the latter stage, the program requires as input the average gain of the multiwire avalanches which cannot be precisely estimated by calculation.

The program generates Cherenkov photons randomly in the radiator along the trajectory of the beam particle with a given energy and incidence angle $\theta_{p}$ relative to the radiator normal. The Cherenkov photon energy $E$ is chosen according to a Frank-Tamm distribution between the energy limits of detector response, and then the Cherenkov angle $\theta$ is determined from the radiator refractive index (Figures $10(\mathrm{a}, \mathrm{b})$ ). The Cherenkov azimuthal angle $\phi$ is, of course, chosen from a flat distribution.

Each generated photon of given energy, direction and polarization is tracked through a given medium of pathlength $z$ and is absorbed and lost with probability $\left(1-e^{-\mu z}\right)$ or transmitted into the next medium with probability $e^{-\mu z}$ (see Figures $11 \mathrm{~b}$ and 12 for $\ell_{\text {abs }}=\mu^{-1}$ ). It may eventually enter the detector gas volume where it is absorbed with probability $\left(1-e^{-\mu z}\right)$ (Figure $8(b)$ for $\ell_{\mathrm{abs}}=\mu^{-1}$ ) and subsequently create a photoelectron with probability $Q$ (Figure 9 ).

The reflection probability at each boundary interface is calculated using the Fresnel relations for the $s$ and $p$ states of polarization and the refractive indices of the media.

Starting from the production point, the photoelectron is tracked along a field line to a wire where the detector response is simulated [2] assuming a Furry distribution of detector gain (before electronic filtering) with a mean multiplication adjusted to the experimental excitation curve (see Figure 17(a)). The induced charge density distribution per unit cathode area, for an anode-cathode distance $d$ at distance $r$ from the the avalanche position,

$$
\rho(r, d)=\exp \left\{-(r / d)^{\alpha+\beta d}\right\}
$$

was adjusted to reproduce the variations of cluster multiplicity with avalanche gain in the current data, yielding the parameter values $\alpha=0.982$ and $\beta=1.00 \mathrm{~mm}^{-1}$ for $d=0.5 \mathrm{~mm}$, differing from the value previously found $(\alpha=0.68)$ [2] because of different front-end electronics.

Following the procedure described in [2], the program generates secondary "feedback" photons from Carbon atomic excitation levels and sums the induced charge per pad to determine, after discrimination, the detection probability, the hit pad addresses, and the cluster multiplicity.

\subsection{Reconstruction Procedure}

The photon detection point is defined as the digital barycenter of hit pads with common sides.

The Cherenkov angle of a detected photon is calculated by the reconstruction program using a method of iteration to reduce either experimental data or MC simulated data. The Cherenkov angles $\theta^{(0)}, \phi^{(0)}$ are calculated to zeroth order by finding the angles between the incident particle direction and the initial photon direction. In zeroth order this is just the vector between the detection point and emission point (assumed to be at the middle of the radiator).

These angles are then further refined by taking into account refraction at the various media boundaries along the photon trajectory in an iterative manner. Starting with the zeroth order angles, the photon is tracked sequentially from the putative emission point through all the media of the Prototype until detection on the cathode plane, using a mean photon energy of $8.32 \mathrm{eV}\left(\mathrm{CaF}_{2}\right)$ or $8.34 \mathrm{eV}(\mathrm{LiF})$ as estimated by Monte Carlo. The distance $\Delta r$ between this traced position on a reference plane (parallel to the cathode plane) and the actual measured position is then calculated. The local derivatives $(d r / d \theta)$ and $(d r / d \phi)$ are numerically evaluated by varying $\theta$ and $\phi$ by $0.5 \mathrm{mrad}$, and used with $\Delta r$ to obtain the first order Cherenkov angles. These values $\theta^{(1)}, \phi^{(1)}$ are then used to start a new photon tracking pass, for which $\Delta r$ is again calculated, and the next order angles found. This algorithm is repeated iteratively until $\Delta r<1$ $\mu \mathrm{m}$. This procedure is fast and efficient, requiring only $2-3$ iterations for convergence on final $\theta$ and $\phi$ values. 
The various contributions to the resolution $\sigma_{\theta}$ are calculated by varying each experimental variable $\left\{x, y, z, z_{e}, E, \theta_{p}, \phi_{p}\right\}$ by one standard deviation. The event Cherenkov angle $\theta_{\text {evt }}$ is calculated by averaging over the $N_{\text {pe }}$ photoelectrons in the event.

To simulate image background, for comparison with experimental data, the MC program generates the Cherenkov light produced by $\delta$-rays created by the incident particle in the radiator and the window.

\subsection{Monte Carlo Calculation of the Photoelectron Yield and Resolution}

Images from $2000 \mathrm{CaF}_{2}$ and $\mathrm{LiF}$ events, generated under conditions identical with that of the corresponding experimental run, are shown in Figures 14(a) and 14(b). The reconstructed single-photon Cherenkov angle distributions in the three sectors and their summation are seen in Figures 15(a) and 15(b) for $4000 \mathrm{CaF}_{2}$ and $4000 \mathrm{LiF}$ events.

A fit to the generated events is made with the following bi-functional form: a Gaussian distribution for the rise-side and a Landau-type Moyal distribution [26] for the tail-side, necessary because of the poor $\chi^{2}$ description obtained with a simple Gaussian. The asymmetric tail of this distribution results from an enhancement of the TEA quantum response at the highest photon energies due to the strongly increasing chromaticity of the radiator media - and is not due to a non-Gaussian aspect of the Cherenkov radiation. The small background comes from the $\delta$-ray contribution.

The parameters of the Gaussian/Moyal bi-functional distribution are extracted as follows. The most probable value of the Cherenkov angle $\bar{\theta}$ is taken as the peak of this function; $\bar{\theta}=886.3$ mrad for $\mathrm{CaF}_{2}$ and $842.9 \mathrm{mrad}$ for LiF. This is not strictly a mean value in the Gaussian sense.

The per photon rms standard deviation is extracted simply as FWHM/2.36 with the fullwidth at half-maximum defined by the function itself; this yields $\sigma_{\theta}=17.1 \mathrm{mrad}$ for $\mathrm{CaF}_{2}$ and $12.3 \mathrm{mrad}$ for $\mathrm{LiF}$, somewhat smaller than the values given by the analytical calculation (18.2 mrad for $\mathrm{CaF}_{2}$ and $14.5 \mathrm{mrad}$ for $\mathrm{LiF}$ ).

The per event photoelectron yield is determined for full detector efficiency by a numerical integration of the area under the bi-function (background subtracted) averaged over the number of events; $N_{\mathrm{pe}}=8.59 \mathrm{pe} / \mathrm{image}$ for $\mathrm{CaF}_{2}$ and $9.39 \mathrm{pe} / \mathrm{image}$ for LiF. Note that these values are about $15 \%$ less than the analytical estimates.

Errors are difficult to assign from this fit procedure; we will quote a general error of $0.5-1.0$

mrad for $\bar{\theta}$ and $\sigma_{\theta}$, and an error on $N_{\text {pe }}$ given by only statistics, viz., $\sqrt{N_{\text {pe }} / N_{\text {evt }}}$ for $N_{\text {evt }}$ events. This bi-functional fit will be our standard fit, applied to both $\mathrm{MC}$ and experimental data.

Bulk absorption of the Cherenkov photons in the media and reflection at the interfaces are calculated by the program, which also automatically accounts for transmission losses in the present design due to geometrical dead areas (i.e., inter-sector mechanical structure, window frames across the $x$-direction, and non-instrumentation of the 15 th column of electronics) $T_{\mathrm{da}}=$ 0.904 ; and the window transmission (after/before) trace metallization $T_{\mathrm{tr}}=0.93$, and hence a total transmission $T=T_{\mathrm{da}} \cdot T_{\mathrm{tr}}=(0.904)(0.93)=0.841$.

The background under the bi-functional curve from $\delta$-rays is small, and contributes to the photoelectron yield only $0.04 \mathrm{pe} / \mathrm{image}$ within the fiducial limits or $0.65 \mathrm{pe} / \mathrm{image}$ in the total detector area.

\section{EXPERIMENTAL RESULTS}

The beam tests were made with $\mathrm{LiF}$ and $\mathrm{CaF}_{2}$ crystal radiators at three different incident angles, as summarized in Table III. Most of the runs (of 2000-10000 events each) were made with a $10 \mathrm{GeV} / c \pi^{-}$beam, except for several with a $2 \mathrm{GeV} / c$ and $6 \mathrm{GeV} / c$ positive beam $\left(\pi^{+}, p\right)$.

During operation of the Prototype, the lever arm volume was flushed with $50 \ell / \mathrm{h}$ of pure Argon gas, and the photon detectors with $10 \ell / \mathrm{h}$ of $\mathrm{CH}_{4}$ bubbled through TEA at $17^{\circ} \mathrm{C}$; otherwise, when not in operation, they were continuously rinsed with Argon gas.

The potentials applied to the window traces were $U_{W}=-1.7 \mathrm{KV}$, and to the anode wires $U_{a}=1.4 \mathrm{KV}$. A detailed study of single photoelectrons in this detector has shown that, with 
TEA as the photosensor, these potentials give full pad detection efficiency at a $\mathrm{DAC}=6$ threshold setting and $30 \mathrm{nsec}$ strobe width [3]. For the present beam tests we used a 50 nsec strobe width; however, in the previously-reported cosmic ray tests [7], the strobe width was chosen to be 100 nsec because of the difficulty of precisely aligning the strobe due to the low cosmic ray rates. An illustration of the strobe delay curves obtained is shown in Figure 16 for a $\mathrm{CaF}_{2}$ radiator. For Sector 0 , the potential $U_{a}$ was turned off in order to observe the pure detector and electronic noise. The curve has a full efficiency plateau of $25-30 \mathrm{nsec}$ width, thus confirming that a $25 \mathrm{nsec}$ strobe width can be used for LHC applications.

The FWHM of this distribution is $80 \mathrm{nsec}$ with a $25-30 \mathrm{nsec}$ flat-top hence a minimum FWHM would be 50 nsec. The $(3 \sigma)$ contribution from the photoelectron dispersion in TEA is $30 \mathrm{nsec}$, the detector pulse rise time dispersion is $15-20 \mathrm{nsec}$ and the clock asynchrony is 20 nsec thus accounting for $50 \mathrm{nsec}$ minimum FWHM. For a CsI photocathode the photoelectron dispersion term vanishes, hence a strobe of $25 \mathrm{nsec}$ should suffice. Note that the delay time plotted in Figure 16 is with respect to the trigger pulse, hence the zero is arbitrary. Recall, however, that the absolute delay provided by the VLSI electronics is $1.32 \mu \mathrm{sec}$ at $50 \mathrm{MHz}$.

Figure 17(a) shows the variation of the photoelectron yield (background subtracted in the image fiducial limits) as a function of the anode wire voltage $U_{a}$, for each of the three sectors and their total. We see indications of a single electron plateau above $U_{a}=1.475 \mathrm{KV}$; thus, in the present tests, by operating the photon detectors $U_{a}=1.4 \mathrm{KV}$, we lost on detection efficiency more than expected from previous tests [7]. This inadequate setting of operating voltage was in fact discovered after data-taking when a more sophisticated off-line analysis was performed as compared to the on-line optimization.

The maximum voltage before sparking is not well-fixed because the probability to get a discharge is a function of operation time and charge particle density. For a short time, the detector can easily hold 1.5 to $1.55 \mathrm{KV}$, therefore we chose $1.4 \mathrm{kV}$ as a safe operating point.

To correct for this $\sim 15 \%$ mean loss in detector efficiency, we used the strong correlation between pad multiplicity and anode voltage $U_{a}$ shown in Figure 17(b). The photoelectron yields versus pad multiplicity plotted in Figure 17 (c) show plateau-like curves similar to Figure 17(a). To compare the photoelectron yields and resolution with the $\mathrm{MC}$ and the analytical estimates, we corrected all clusters to be on plateau, i.e., to a pad multiplicity of 1.5 . In Table IV we list both the measured $\left(N_{\mathrm{pe}}\right)$ and voltage-corrected $\left(N_{\mathrm{pe}}^{\mathrm{corr}}\right)$ photoelectron yields.

\subsection{Experimental Results for the $\mathbf{1 0} \mathrm{mm} \mathrm{LiF} \mathrm{Radiator}$}

\subsubsection{Experimental Signal and Backgrounds}

Figures 18 to 21 show the raw data obtained in two typical runs of 2000 events each with LiF radiator \#2 at $\theta_{p}=25^{\circ}$. Two scatter plots are displayed in Figure 18, one with and one without TEA in $\mathrm{CH}_{4}$.

Here, the Cherenkov image falls between two ceramic spacers so that the only photon losses are due to the window traces $\left(T_{\mathrm{tr}}=0.93\right)$ and the dead area due to transverse window frames and the non-instrumented 15 th columns $\left(T_{\mathrm{da}}=0.904\right)$. One may also observe some dead pads at the edge of the beam spot and in the image region. We note from the scatter plot that the noise is small. The faint image still seen without TEA in Figure 18(b) is certainly due to TEA vapor which had been adsorbed on the detector surfaces and not yet completely removed by rinsing. That this image is faint is proven by Figures 19(a) (with TEA) and 19(b) (without TEA) where the number of hits per row are plotted on the same scale. Below the images (i.e., row addresses less than 80 ) the very low (negligible) background count comes mostly from electronic noise but above it is mostly from scintillation (see $\S 7.2 .1$ ).

The per event distribution of hit pads and clusters without fiducial cuts are shown in Figures 20(a,b) respectively, where the shaded (open) histograms are with (without) TEA. With TEA the mean number of pads/cluster are 1.41, 1.69 and 1.27 for Sectors 0,1 , and 2, respectively. The value in Sector 1 includes the charged particle pad multiplicity which is 3.8 pads/cluster. 
The number of hit pads per sector with (without) TEA are $5.58(0.38), 13.84(3.01)$ and $3.92(0.26)$ for Sectors 0,1 , and 2 , hence even without pattern selection, the $S / N$ ratios are high, i.e., $14.8,4.60$ and 15.1 (the ratio in Sector 1 is lower because of the pad hits from the charged particle). Thus, the per event image quality is excellent as can be seen in Figure 21 where four average events are shown.

\subsubsection{Image Reconstruction, Cherenkov Angle Resolution, and Photoelectron Statistics}

Figures 22 to 26 summarize results obtained from the analysis of 11805 reconstructed images from 18000 triggers (MWPC track acceptance efficiency $\sim 66 \%$ ). In Figure 22 the images have been projected onto a plane normal to the beam direction via the transformation

$$
\begin{aligned}
& x=D \tan \theta \cos \phi=R \cos \phi \\
& y=D \tan \theta \sin \phi=R \sin \phi,
\end{aligned}
$$

where $x, y$ are the scatter plot coordinates, $D=Z / \cos \theta_{p}$ is the distance to the fictitious cathode-pad plane, $Z$ is the lever-arm, $R=D \tan \theta$ is the projected ring radius, and $\theta, \phi$ are the reconstructed polar and azimuthal Cherenkov angles. The beam spot is clearly visible at plot coordinates $(x=2.2 \mathrm{~cm}, y=0 \mathrm{~cm})$. However, the true hit point in this coordinate system is at the origin, but when this point is reconstructed as if it were a Cherenkov photon, it appears at $(2.2,0) \mathrm{cm}$. Note that the radius $R \approx 17 \mathrm{~cm}$ agrees well with $R=Z \tan \theta / \cos \theta_{p}$ for the known parameters $Z=13.9 \mathrm{~cm}, \theta=836 \mathrm{mrad}$, and $\theta_{p}=25^{\circ}$. Note also that this projection removes all refraction effects at the media interfaces and exhibits the image for a fictional detector at distance $D$; however all the real sources of error remain.

Figure 23 shows the image radii for each of the three sectors as well as the beam spot in Sector 1 . The image spread around the beam spot comes from scintillation in the window (as will be discussed in $\S 7.2 .1$ ). The small amount of residual background comes from scintillation by the charged particle in the radiator and Cherenkov radiation produced by $\delta$-rays.

The scatter plot in Figure 24 of $\theta$ vs. $\phi$ indicates that the azimuthal distribution of detected photoelectrons is flat, as expected from theory.

The reconstructed single-photon Cherenkov angle distributions for each of the individual sectors and their sum are shown in Figure 25. Our standard bi-functional Gaussian/Moyal form was used to fit these distributions and the parameters extracted (as described in $\S 6.4$ for the MC simulated data). The reduced chi-square $\chi^{2} /$ dof of the fit is 0.22 , with CL $>99 \%$, hence quite good. The apparent variation of the mean (most probable) Cherenkov angle $\bar{\theta}$ (i.e., the peak) from sector to sector is about $0.25 \%$. The resolution calculated from the FWHM is $\sigma_{\theta} \approx 13.8 \mathrm{mrad}$, and is about the same for each of the three sectors. The mean number of detected photoelectrons per event (background subtracted) is $N_{\mathrm{pe}}=10.4 \mathrm{pe} / \mathrm{image}$ for the full $\operatorname{LiF}\left(\theta_{p}=25^{\circ}\right)$ data-set. However, one observes an asymmetry between Sectors 0 and 2 of about $30 \%$, due of the inadequate voltage setting. This asymmetry vanishes almost completely when the clusters are corrected (as described in $\S 7$ ) to be on plateau, i.e., to 1.5 hit pads per cluster, and then $N_{\mathrm{pe}}^{\text {corr }}=11.2$, hence a correction of $7.7 \%$.

Figure 26(a) shows a scatter plot of the (background subtracted) photoelectron yield $N_{\text {pe }}$ $v s$. the mean per event Cherenkov angle $\theta_{\text {evt }}$, displaying its constancy, but of course with a larger variance at low $N_{\text {pe }}$. The $N_{\text {pe }}$ distribution shown in Figure 26(b) is Poissonienne, with a large enough mean to appear Gaussian $\left(\bar{N}_{\mathrm{pe}}=9.2 \mathrm{pe} / \mathrm{image}\right.$ for events with $N_{\mathrm{pe}}>1$ and $\left.\bar{\theta} \pm 2 \sigma_{\theta}\right)$. The mean per event Cherenkov angle is $\bar{\theta}_{\text {evt }}=838.8 \mathrm{mrad}$ (for events with $N_{\mathrm{pe}}>1$ and a $2 \sigma_{\theta}$ cut around $\bar{\theta}$ ). as shown in Figure 26(c) with the per event variance $\sigma_{\theta_{\text {evt }}}=4.5 \mathrm{mrad}$, scaling as $\sigma_{\theta} / \sqrt{N_{\mathrm{pe}}}$ as expected.

\subsection{Experimental Results for the $10 \mathrm{~mm} \mathrm{CaF}{ }_{2}$ Radiator}

\subsubsection{Experimental Signal and Backgrounds}

Figures 27 to 29 show the raw data of the runs taken with $\mathrm{CaF}_{2}$ radiator $\# 3$ at $\theta_{p}=30^{\circ}$ in conditions appropriate to study the origin of the background, however small. 
The scatter plot of the hit pads shown in Figure 27 represents 5000 superimposed events with (a) the radiator in the beam line, and (b) the radiator out of the beam line. In (a), the Cherenkov signal and the beam impact regions are clearly evident as is the scintillation background. That the latter comes mostly from scintillation in the radiator is proven by the shadow of the detector window frame seen around row address 50 (also near 105). In (b), however, we see the "no radiator" beam spot and only the much less intense scintillation from the $\mathrm{CaF}_{2}$ window.

The distribution of the number of clusters per event for Sectors 0, 1, and 2 are shown in Figure 28(a). For the empty radiator run, shown in Figure 28(b), the clusters/event values are compatible with electronic noise in Sectors 0 and 2. For Sector 1, assuming one cluster per beam track and the same level of electronic noise (0.7), there remains a background 0.6 clusters/event due to scintillation in the window, which disappears when TEA is removed.

Figure 29 shows the projections of the hit pads, giving a row-wise $(x)$ profile of Sector 1 only. In Figures 29(a) and 29(b) data were taken with the same $\mathrm{CaF}_{2}$ radiator \#3 at $30^{\circ}$, but for two different positions of the beam impact point, at row addresses 94 or 100 . One can observe that, in both figures, the background distribution under the beam spot remains the same between row addresses 78 and 104, which correspond to the extremities of the impacted CaF2 window. The profile in Figure 27(c) was obtained for the "no radiator" run when the beam impacted the $\mathrm{CaF}_{2}$ window at row address 37 . It shows a background of about the same intensity and shape but centered between row addresses 26 and 52, i.e., the edges of this $\mathrm{CaF}_{2}$ window.

These observations prove unambiguously that this background is created by photoconversion of scintillation light generated by the charged particle in the window. The light escapes from the crystal into the detector either directly, or for the fraction of the light internally trapped, by scattering from the unpolished edges.

Emission of scintillation light in the radiator is also visible in Figure 30 (a large event sample from a LiF radiator) which shows the scatter plot of reconstructed clusters. One can easily see background-free regions around row addresses 101, 118, 136 and 152 with widths which monotonically increase with the distance from the beam spot. Simulation of isotropic light emission from tracks in the radiator shows that this topology is well reproduced by the shadowing effect of the window frame.

All the tests discussed above confirm that scintillation in the radiator is the primary (but still small) source of background, however the absolute scintillation light yield has not been estimated. Similar results were obtained with other radiators.

\subsubsection{Image Reconstruction, Cherenkov Angle Resolution, and Photoelectron Statistics}

For comparison with the above LiF radiator data, Figure 31 shows 3822 superposed images from $\mathrm{CaF}_{2}$ radiator \#3, reconstructed and projected onto the (fictitious) plane normal to the beam axis defined by Equations (9). This scatter plot clearly exhibits, as before, the contribution of scintillation around the beam spot, the good signal-to-noise ratio within the fiducial limits, and the sharp front edge and less sharp trailing edge of the images.

Figure 32 shows the reconstructed single-photon Cherenkov angle distributions for the individual sector and their sum. The standard bi-functional fit parameters were determined as before.

The most probable Cherenkov angle is $\bar{\theta}=878.2 \mathrm{mrad}$ with a sector to sector variation of $\sim 0.4 \%$. The resolution, calculated from the FWHM, is $\sigma_{\theta}=17.0 \mathrm{mrad}$, again varying somewhat from sector to sector. The background subtracted photoelectron yield per event is $N_{\text {pe }}=7.45$ for the full data-set. However one observes an asymmetry between Sectors 0 and 2 due to the low voltage setting. This asymmetry almost vanishes when the data are corrected (as described in $\S 7$ ) to 1.5 hit pads per cluster, giving then $N_{\mathrm{pe}}^{\text {corr }}=9.88$, hence a worse-case correction of $33 \%$.

Figure 33(a) shows a scatter plot of (background subtracted) photoelectron yield $N_{\text {pe }} v s$. the Cherenkov angle per event $\theta_{\text {evt }}$, for the full $\mathrm{CaF}_{2} \# 3 \theta_{p}=30^{\circ}$ data sample, displaying its constancy but of course with a larger variance at low $N_{\mathrm{pe}}$. The $N_{\mathrm{pe}}$ distribution shown in 
Figure 33(b) is Poissonienne with $\bar{N}_{\mathrm{pe}}=6.7$ (for events with $N_{\mathrm{pe}}>1$ and a $2 \sigma_{\theta}$ cut around $\bar{\theta}$ ). The mean Cherenkov angle per event $\bar{\theta}_{\text {evt }}=885.2 \mathrm{mrad}$ (for $N_{\mathrm{pe}}>1$ and a $\bar{\theta} \pm 2 \sigma_{\theta}$ ). as shown in Figure $33(\mathrm{c})$, with a per event variance $\sigma_{\theta_{\text {evt }}}=6.7 \mathrm{mrad}$, and as expected it scales as $\sigma_{\theta} / \sqrt{N_{\mathrm{pe}}}$.

\section{DISCUSSION OF EXPERIMENTAL RESULTS}

Table IV summarizes the experimental results obtained in the beam tests of the Prototype with four different crystal radiators and three incident angles.

The voltage-corrected $\mathrm{LiF}$ data $\left(N_{\mathrm{pe}}^{\text {corr }}, \sigma_{\theta}, \sigma_{\theta_{\mathrm{evt}}}=\sigma_{\theta} / \sqrt{N_{\mathrm{pe}}^{\text {corr }}}\right)$ versus $\theta_{p}$ are shown in Figure 34 along with the analytic calculations of the same quantities. As may be seen, the magnitudes of $\sigma_{\theta}$ and $N_{\mathrm{pe}}^{\text {corr }}$ along with their $\theta_{p}$ dependence agree well with the analytic expectations. Similarly, the corrected $\mathrm{CaF}_{2}$ data are shown in Figure 35. Here, only at $\theta_{p}=30^{\circ}$ do we have have unobstructed (reliable) images for both radiators (\#3 and \#5) with the values of $\sigma_{\theta}$ and $N_{\text {pe }}^{\text {corr }}$ again in accord with the analytic estimates.

However we note from Table IV that the MC estimates of $N_{\text {pe }}$ are about $16 \%$ lower than the experiment and the analytic estimates. This may indicate that the TEA quantum efficiency is really $16 \%$ too low or that possibly the $\mathrm{CaF}_{2}$ window absorption is lower than calculated using transmission measurements (or a combination of the two).

Holroyd et al. [19] give a $20 \%$ error on their absolute scale for both the TEA and TMAE quantum efficiencies and since our calibration of TEA was relative to TMAE, we depend on the Holroyd calibration.

On the other hand, at average photon energy of $8.32 \mathrm{eV}$, the measured $\mathrm{CaF}_{2}$ radiator absorption coefficient $0.25 \mathrm{~cm}^{-1}$ but the measured $\mathrm{CaF}_{2}$ window absorption coefficient is 0.48 $\mathrm{cm}^{-1}$. Since these crystals were ordered at the same time from the same source, we were surprised by this difference. In the process of vacuum deposition of the metallic HV traces, the windows were subjected to prolonged exposure to air and could have absorbed significant quantities of water which has strong absorption in the $8.32 \mathrm{eV}$ region. The window absorption coefficients of Figure 11(b) were measured immediately after vacuum deposition and it is conceivable that after prolonged ( $\sim 1$ year) flow of zero humidity gas through the photon detector and lever arm, that the water level was reduced to that of radiator $\mathrm{CaF}_{2}$ (which was practically not exposed to air). In fact, for the average path length of $0.4 \mathrm{~cm}$ in the detector window, the attenuation difference would just give the needed $10 \%$ increase of photons reaching the detector. We cannot, of course, be sure of this hypothesis unless we remove (unglue) a window and remeasure its transmission.

A combination of these effects, increasing the photoelectron yield by $16 \%$, is required to bring the Monte Carlo calculation into agreement with experiment. The effect of photon overlap, shown by MC simulation in Figure 36(a), causes a additional loss of photoelectrons in both experimental and MC simulated data. In the conditions of these tests (3-5 clusters/sector), $\mathrm{MC}$ calculation shows that about $10 \%$ of the photons are lost due to overlap, even with our low pad multiplicity $(\sim 1.5)$ and small pixels. Clearly, larger pad multiplicities or pixel sizes would be detrimental. This is proven by Figure 36(b) where the loss in angle resolution due to photon overlap is shown for both experimental and $\mathrm{MC}$ simulated data $(1.2 \mathrm{mrad}$ for 4 clusters/sector). Factoring in the $10 \%$ loss on $N_{\text {pe }}$ we conclude that $\sigma_{\theta_{\text {evt }}}=\sigma_{\theta} / \sqrt{N_{\text {pe }}}$ is increased by $\sim 15 \%$ due to photon overlap.

Because of photon overlap, both the experimental and MC photoelectron yields are decreased by $10 \%$, but the $16 \%$ difference remains. Increasing the quantum efficiency (and/or window transmission) by $16 \%$ would bring $\mathrm{MC}$ in agreement with data and only marginally destroy the almost-perfect agreement between the analytical calculations and experiment (q.v. Table IV and Figures 34 and 35). This is so because the analytical calculations do not include the $10 \%$ photon overlap loss, hence a $16 \%$ increase of quantum efficiency would mean that the analytical values would be $6 \%$ higher than then experiment (i.e., within the overall errors of these data). 
According to the standard equation $[9,10]$, the momentum at which particles of mass $m_{1}$ and $m_{2}$ are separated by $n_{\sigma}$ standard deviations is

$$
p_{m_{1} / m_{2}}\left(n_{\sigma}\right)=\left[\frac{m_{1}^{2}-m_{2}^{2}}{2 n_{\sigma} k_{R}}\right]^{1 / 2},
$$

where $k_{R}$ is the RICH constant

$$
k_{R}=\sigma_{\beta} / \beta^{2}=\tan \theta\left(\sigma_{\theta} / \sqrt{N_{\mathrm{pe}}}\right)=\tan \theta \sigma_{\theta_{\mathrm{evt}}} .
$$

The corrected experimental values of Table IV correspond to $k_{R}=4.56 \times 10^{-3}(\mathrm{LiF})$ and $6.51 \times 10^{-3}\left(\mathrm{CaF}_{2}\right)$, hence the momenta for $3 \sigma \pi / K$ separation are $p_{\pi / K}(3 \sigma)=2.86 \mathrm{GeV} / c$ $(\mathrm{LiF})$ and $2.39 \mathrm{GeV} / c\left(\mathrm{CaF}_{2}\right)$. The experimentally achieved merit factors, after correction for azimuthal angle acceptance, are $N_{0}=65.5 \mathrm{~cm}^{-1}(\mathrm{LiF})$ and $57.7 \mathrm{~cm}^{-1}\left(\mathrm{CaF}_{2}\right)$, compared to 53.8 $\mathrm{cm}^{-1}$ and $50.2 \mathrm{~cm}^{-1}$ respectively from the Monte Carlo calculation.

An illustration of the particle separation at $2 \mathrm{GeV} / \mathrm{c}$ particle beam is shown in Figures 37 and 38 . The results were obtained with the LiF radiator at $25^{\circ}$ with a positive hadron beam, containing both $\pi^{+}$and $p$. Figure 37 exhibits clearly the concentric rings from both protons and pions for 292 superimposed images, projected onto the plane perpendicular to the beam direction via the transformation of Equations (9).

In Figure 38 is shown a scatter plot of the mean Cherenkov angle per event $\theta_{\text {evt }}$ versus the (background subtracted) photoelectron yield $N_{\text {pe }}$, as well as its projections, for the $2 \mathrm{GeV} / c$ run. The distance between peaks is $91 \mathrm{mrad}$ and the per event resolution $\sigma_{\theta_{\text {evt }}}=4.4 \mathrm{mrad}$ (see Figure 26) hence their separation is 20.7 standard deviations in rather good agreement with 23.6 standard deviations predicted from Equation (11) with the LiF RICH constant given above.

It was originally foreseen to take beam data at several different values of the particle momentum. Unfortunately, the tests ended prematurely due to an accident, which happened during a standby period, involving the high voltage supply modules.

The problem arose during a thunderstorm, when, although the front panel control switches on the HV supplies were off (standby, hence the high voltages OFF), a malfunction of the NIM mother crate caused the high voltages to switch ON. In fact, because of this crate problem, the high voltages repeatedly increased without limitation up to breakdown in the detectors (MWPCs and Prototype) all during the night of the storm.

We want to stress that as a result of this accident the aluminized mylar cathode planes of the MWPCs were entirely destroyed and all wire resistors were fried, but in the Fast-RICH Prototype only one wire in the three sectors was broken. Moreover, no protected electronic channels were lost. We report here this accident as an inadvertent test of the robustness of this detector, an important characteristic for large detectors of $10-20 \mathrm{~m}^{2}$ as now envisioned for $B$-Factory Colliders. 


\section{CONCLUSIONS}

We have demonstrated the operation of a full scale Fast-RICH detector system for particle identification. The performance of our Prototype is summarized in Table V. With a LiF radiator and TEA gaseous photosensor we can attain $\pi / K$ identification up to $p_{\pi / K}(3 \sigma)=2.86 \mathrm{GeV} / c$. The achieved merit factor, after correction for azimuthal angle acceptance, is $N_{0}=65.5 \mathrm{~cm}^{-1}$ corresponding to $N_{\text {pe }}=11.2$ photoelectrons per image for a $10 \mathrm{~mm}$ thick LiF radiator, even with a $\sim 10 \%$ photoelectron loss due to the limited detector area $\left(\sim 0.5 \mathrm{~m}^{2}\right)$ and the $16 \%$ opacity loss. The performance of the detector for $\sigma_{\theta}$ agrees within error with the Monte Carlo simulation but $N_{\text {pe }}$ is $16 \%$ larger than expected, indicating that the quantum efficiency of TEA (Figure 9) or the window transparency (Figures 9 and 11) must be correspondingly increased. Limitations in the detector performance due to photon overlap have been demonstrated. 


\section{ACKNOWLEDGEMENTS}

We would like to thank J. Haïssinski and J.P. Repellin of IN2P3 and Prof. M. Froissart of College de France for their continuous support and interest in the Fast-RICH detector development. The competence and enthusiasm of Prof. P. Sharp and his collaborators M. French and M. Lovell of the RAL Microelectronics group made this project a success. We are very much indebted to them for the quality of their work, and we want to stress that it was a great pleasure to collaborate with them. The support of Prof. Zichichi of CERN-LAA and Profs. Takasaki and Iwata at KEK in Japan was important. We wish to thank them for their comprehensive help. J. Eggert and K. Gabathuler of PSI and their engineer and technician G. Heinen and L. Meier have efficiently contributed to the first stages of the development, especially on the construction. We are aware of the excellent quality of their work and are grateful. Several other competent engineers and technicians have contributed to the construction of the Prototype, especially E. Christophel, F. Lott, G. Hauer, R. Jaeg at CRN (Strasbourg), A. Gandi, D. Berthet of the CERN/MT division, and G. Geydet of the CERN/AT division. Y. Perrin of the CERN/ECP-DS division helped us to install the data acquisition program SPIDER. K. Bätzner and L. Durieu of the CERN/PS-PA team made easier the operation of the PS T9 test beam, and made useful calculations for our beam tune. We thank them very much for their efficient and sympathetic collaboration. Thanks again to C. Joram of Karlsruhe University who made MC calculations to estimate the background from $\delta$-rays, and provided us with refractive index data. One of us (RJM) would like to thank the U.S. National Science Foundation for support, and M. Artuso and S. Stone of Syracuse University for useful discussions. 


\section{REFERENCES}

[1] "Proposal for an Electron Positron Collider for Heavy Flavor Particle Physics and Synchrotron Radiation", PR 88-09, Paul Scherrer Institute (PSI), Villigen, Switzerland (July 1988).

[2] R. Arnold, Y. Giomataris, J.L. Guyonnet, A. Racz, J. Séguinot, and T. Ypsilantis: Nucl.Instr.Meth. A314,465-494 (1992).

[3] M. French, M. Lovell, E. Chesi, A. Racz, J. Séguinot, T. Ypsilantis, R. Arnold, J.L. Guyonnet, J. Egger, and K. Gabathuler: Nucl.Instr.Meth. A324,511-534 (1993).

[4] M.J. French, M. Lovell, and P. Murray: IEEE Trans.Nucl.Sci. NS-40 (1993).

[5] M. French, M. Lovell, E. Chesi, A. Racz, J. Séguinot, T. Ypsilantis, R. Arnold, J.L. Guyonnet, J. Egger, and K. Gabathuler: in Proceedings of the International Conference on Electronics for Future Colliders, LeCroy Research Corporation, Chesnut Ridge, New York, May 1993, p. 105.

[6] M. French, M. Lovell, E. Chesi, A. Racz, J. Séguinot, T. Ypsilantis, R. Arnold, J.L. Guyonnet, J. Egger, and K. Gabathuler: Nucl.Instr.Meth. A343,222-230 (1994).

[7] J.L. Guyonnet, R. Arnold, J.P. Jobez, J. Séguinot, T. Ypsilantis, E. Chesi, A. Racz, J. Egger, K. Gabathuler, C. Joram, I. Adachi, R. Enomoto, T. Sumiyoshi: Nucl.Instr.Meth. $\underline{A 343}, 178$ 191 (1994).

[8] J. Séguinot and T. Ypsilantis: Nucl.Instr.Meth. $\underline{A 343}, 1-29$ (1994).

[9] T. Ypsilantis and J. Séguinot: Nucl.Instr.Meth. A343,30-51 (1994).

[10] J. Séguinot: "Les Compteurs Cherenkov : Applications et Limites pour l'Identification des Particules. Développements et Perspectives", CERN-EP/89-92, LPC/89-25 (July 1989), and in Comptes Rendus de l'Ecole Joliot Curie de Physique Nucléaire, Maubuisson, France, September 1988, Les Éditions de Physique, Paris, 1989, p.249.

[11] T. Ypsilantis: "Particle Identification at Hadron Colliders", CERN-EP/89-150 (Nov 1989), and in Proceedings of the Symposium on Particle Identification in High Luminosity Hadron Colliders, Fermilab, 1989, p.133.

[12] J. Séguinot, G. Charpak, Y. Giomataris, V. Peskov, J. Tischhauser, and T. Ypsilantis: Nucl.Instr.Meth. A297,133-147 (1990).

[13] R. Baur et al:: Nucl.Instr.Meth. A343,87-98 (1994).

[14] A. Braem et al.: Nucl.Instr.Meth. A343,163-172 (1994).

[15] C. Joram: Nucl.Instr.Meth. A343,246-249 (1994).

[16] A. Racz: "Système rapide de lecture électronique intǵreé pour détecteurs à structure matricielle. Application à l'imagerie Cherenkov pour l'indentification des particules", These de Doctorat, Universite Paris VI, Juin 1992.

[17] Y. Perrin: "Multi-Tasking SPIDER", CERN-ECP/DS, CERN-MMCNL 31 (July 1991); and J. Peterson: "A VMEbus/OS-9 based Data Acquisition System, CERN-ECP/DS, CERNMMCNL 31 (July 1991).

[18] D.F. Anderson: Nucl.Instr.Meth. A270,416 (1988).

[19] R.A. Holroyd et al.: Nucl.Instr.Meth. A261,446 (1987).

[20] H.H. Li: J.Chem.Phys.Ref.Data. 5(2),329 (1976); (data-sets 7, 28 from internal references 34,28 chosen).

[21] H.H. Li: J.Chem.Phys.Ref.Data. 9(1),161 (1980); (data-sets 2, 14 from internal references 29,41 chosen).

[22] Landolt-Börnstein, Gr.6. Auflage, Bd. II/8, pp.2-405-2-432.

[23] P. Laporte and J.L. Subtil: J.Opt.Soc.Am. 72,1558 (1982).

[24] M. Hempstead, J. Chauveau, G. Comby, J. Séguinot and T. Ypsilantis: Nucl.Instr.Meth. A306, 207-211 (1991).

[25] I.H. Malitson: Appl.Optics 2,1103 (1963).

[26] J.E. Moyal: Phil.Mag. $\underline{46}, 263$ (1955). 
Table I. Comparison Between Experimental Results, Monte Carlo Predictions (MC), and Analytical Calculations (AC) for Cosmic Ray Tests.

\begin{tabular}{||lc|l||c|ccc||}
\hline \hline Radiator & $\theta_{p}$ & $\begin{array}{l}\text { Type of } \\
\text { Result }\end{array}$ & $\begin{array}{c}\text { Reconstr. } \\
\text { Images }\end{array}$ & $\begin{array}{c}\bar{\theta} \\
{[\mathrm{mrad}]}\end{array}$ & $\begin{array}{c}\sigma_{\theta} \\
{[\mathrm{mrad}]}\end{array}$ & $\begin{array}{c}N_{\text {pe }} \\
\text { [pe/image] }\end{array}$ \\
\hline \hline \multirow{2}{*}{$\mathrm{CaF}_{2}$} & $25^{\circ}$ & Expt & 233 & $887.3 \pm 0.5$ & $19.1 \pm 0.4$ & $7.7 \pm 0.2$ \\
& & $\mathrm{MC}$ & 1000 & 885.2 & 19.3 & 6.4 \\
& & $\mathrm{AC}$ & - & 886.8 & 19.7 & 6.2 \\
\hline \multirow{2}{*}{$\mathrm{LiF}$} & $25^{\circ}$ & Expt & 131 & $845.3 \pm 0.6$ & $15.2 \pm 0.5$ & $6.9 \pm 0.2$ \\
& & $\mathrm{MC}$ & 1000 & 836.4 & 13.2 & 7.5 \\
& & $\mathrm{AC}$ & - & 836.0 & 12.2 & 7.3 \\
\hline \hline
\end{tabular}

Table II. Sellmeier Fits for $\mathrm{CaF}_{2}$ and LiF Refractive Indices in the UV Spectral Region.

\begin{tabular}{||l|l||c|cccc||}
\hline \hline $\begin{array}{l}\text { Radiator } \\
\text { Material }\end{array}$ & Fit & $F(E)$ & $\begin{array}{c}F_{1} \\
{\left[\mathrm{eV}^{2}\right]}\end{array}$ & $\begin{array}{c}E_{1} \\
{[\mathrm{eV}]}\end{array}$ & $\begin{array}{c}F_{2} \\
{\left[\mathrm{eV}^{2}\right]}\end{array}$ & $\begin{array}{c}E_{2} \\
{[\mathrm{eV}]}\end{array}$ \\
\hline \hline \multirow{2}{*}{$\mathrm{CaF}_{2}$} & This work & $\left(\frac{n^{2}-1}{n^{2}+2}\right)$ & $17.301 \pm 0.021$ & $12.992 \pm 0.004$ & $464.25 \pm 0.45$ & $54.705 \pm 0.026$ \\
& Malitson $[25]$ & $\left(n^{2}-1\right)$ & 71.855 & 12.350 & 345.36 & 24.667 \\
& Li [21] & $\left(n^{2}-1.33973\right)$ & 122.304 & 13.2264 & $4.11003 \times 10^{-4}$ & $5.85384 \times 10^{-2}$ \\
\hline \multirow{2}{*}{$\mathrm{LiF}$} & This work & $\left(\frac{n^{2}-1}{n^{2}+2}\right)$ & $6.865 \pm 0.175$ & $12.968 \pm 0.037$ & $101.76 \pm 0.75$ & $22.781 \pm 0.081$ \\
& Li [20] & $\left(n^{2}-1\right)$ & 261.495 & 16.8091 & $9.96151 \times 10^{-3}$ & $3.78116 \times 10^{-2}$ \\
\hline \hline
\end{tabular}

Table III. Summary of Beam Tests Performed with Different Radiators.

\begin{tabular}{|c|c|c|c|c|c|c|}
\hline \multirow[b]{2}{*}{$\theta_{p}$} & \multicolumn{6}{|c|}{ - Radiator - } \\
\hline & None & $\mathrm{CaF}_{2} \# 1$ & $\overline{\mathrm{LiF} \# 2}$ & $\mathrm{CaF}_{2} \# 3$ & $\mathrm{LiF} \# 4$ & $\mathrm{CaF}_{2} \# 5$ \\
\hline $20^{\circ}$ & & & & A & $\overline{\phi, \diamond}$ & \\
\hline $25^{\circ}$ & & & $\phi, \diamond$ & & & \\
\hline $30^{\circ}$ & \$ & & $\phi$ & $\bar{\phi}, \varnothing$ & $\overline{4}$ & $\phi$ \\
\hline
\end{tabular}

Note: $=$ runs with TEA; $\diamond=$ runs without TEA; $\diamond=$ runs with variation in $U_{a}$ and DAC. 
Table IV. Comparison Between Experimental Results, Monte Carlo Predictions (MC), and Analytical Calculations (AC) for Beam Tests.

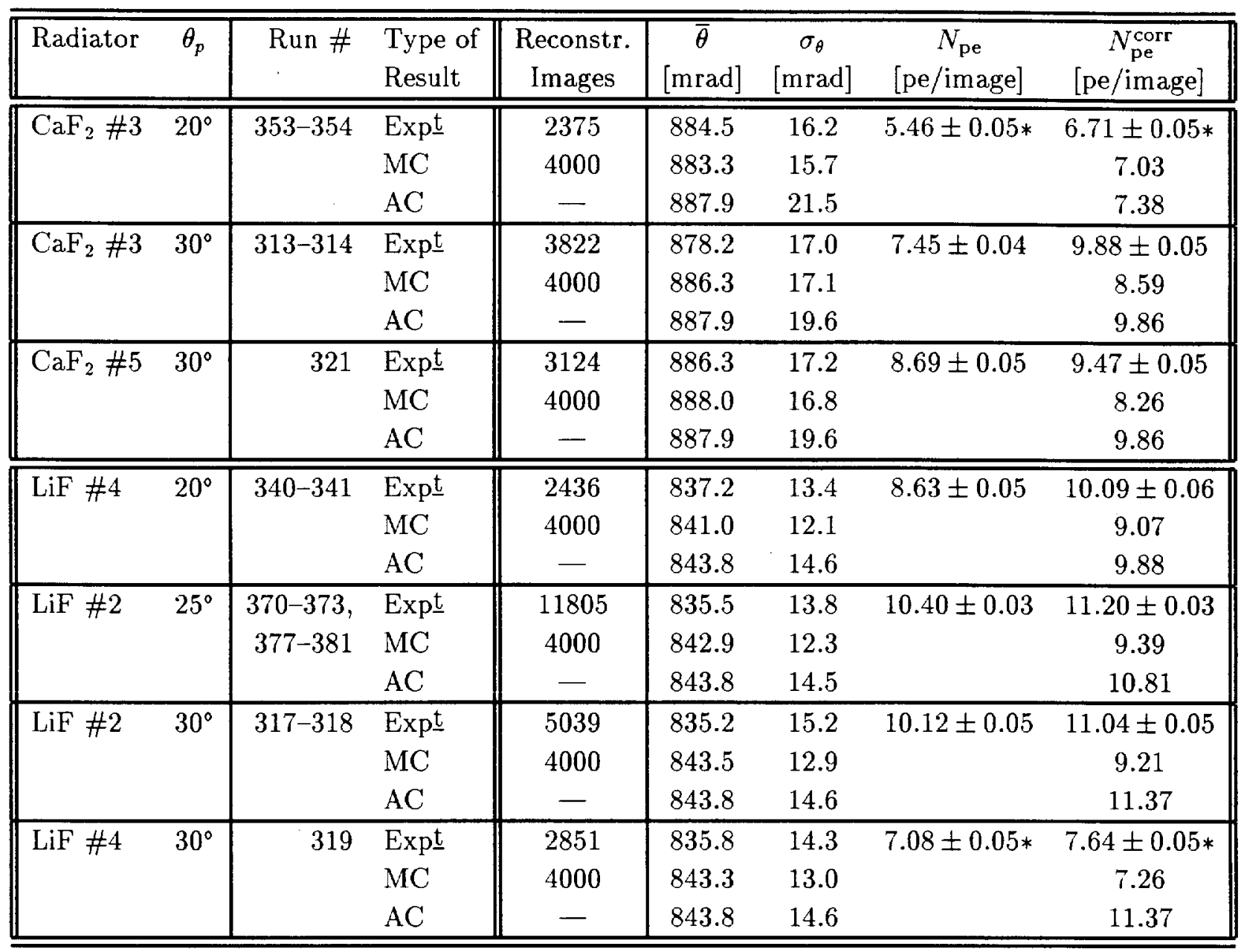

Notes: (1) The errors on $\bar{\theta}$ and $\sigma_{\theta}$ are of the order $0.5-1.0 \mathrm{mrad}$; errors on $N_{\text {pe }}$ are statistical only. (2) An asterisk (*) denotes that in Runs 319 and 353-354, the ring image overlapped the window frame, and so the pe yield is proportionately lowered. (3) Only the experimental values for $N_{\text {pe }}$ are voltage-corrected, and these should be compared with the (uncorrected) $\mathrm{MC}$ and $\mathrm{AC}$ values.

Table V. Summary of Experimentally-Measured and Theoretically-Calculated Fast-RICH Prototype Detector Performance Parameters.

\begin{tabular}{||lc|c||c|ccc|c|c||}
\hline \hline Radiator & $\theta_{p}$ & Run \# & $\begin{array}{c}L \\
{[\mathrm{~cm}]}\end{array}$ & $\begin{array}{c}\left(N_{0}\right)_{\exp } \\
{\left[\mathrm{cm}^{-1}\right]}\end{array}$ & $\begin{array}{c}\left(N_{0}\right) \mathrm{MC} \\
{\left[\mathrm{cm}^{-1}\right]}\end{array}$ & $\begin{array}{c}\left(N_{0}\right)_{\mathrm{AC}} \\
{\left[\mathrm{cm}^{-1}\right]}\end{array}$ & $k_{R}$ & $\begin{array}{c}p_{\pi / K}(3 \sigma) \\
{[\mathrm{GeV} / c]}\end{array}$ \\
\hline \hline $\mathrm{LiF} \# 2$ & $25^{\circ}$ & $370-381$ & 1.103 & 65.5 & 53.8 & 62.3 & $4.56 \times 10^{-3}$ & 2.86 \\
\hline $\mathrm{CaF}_{2} \# 3$ & $30^{\circ}$ & $313-314$ & 1.155 & 57.7 & 50.2 & 56.7 & $6.51 \times 10^{-3}$ & 2.39 \\
\hline \hline
\end{tabular}

Note: All $N_{0}$ values have been corrected for azimuthal angle acceptance ( 3.6 for $\mathrm{LiF}, 4.0$ for $\mathrm{CaF}_{2}$ ). $N_{0}$ is a $\theta_{p}$-dependent quantity. 


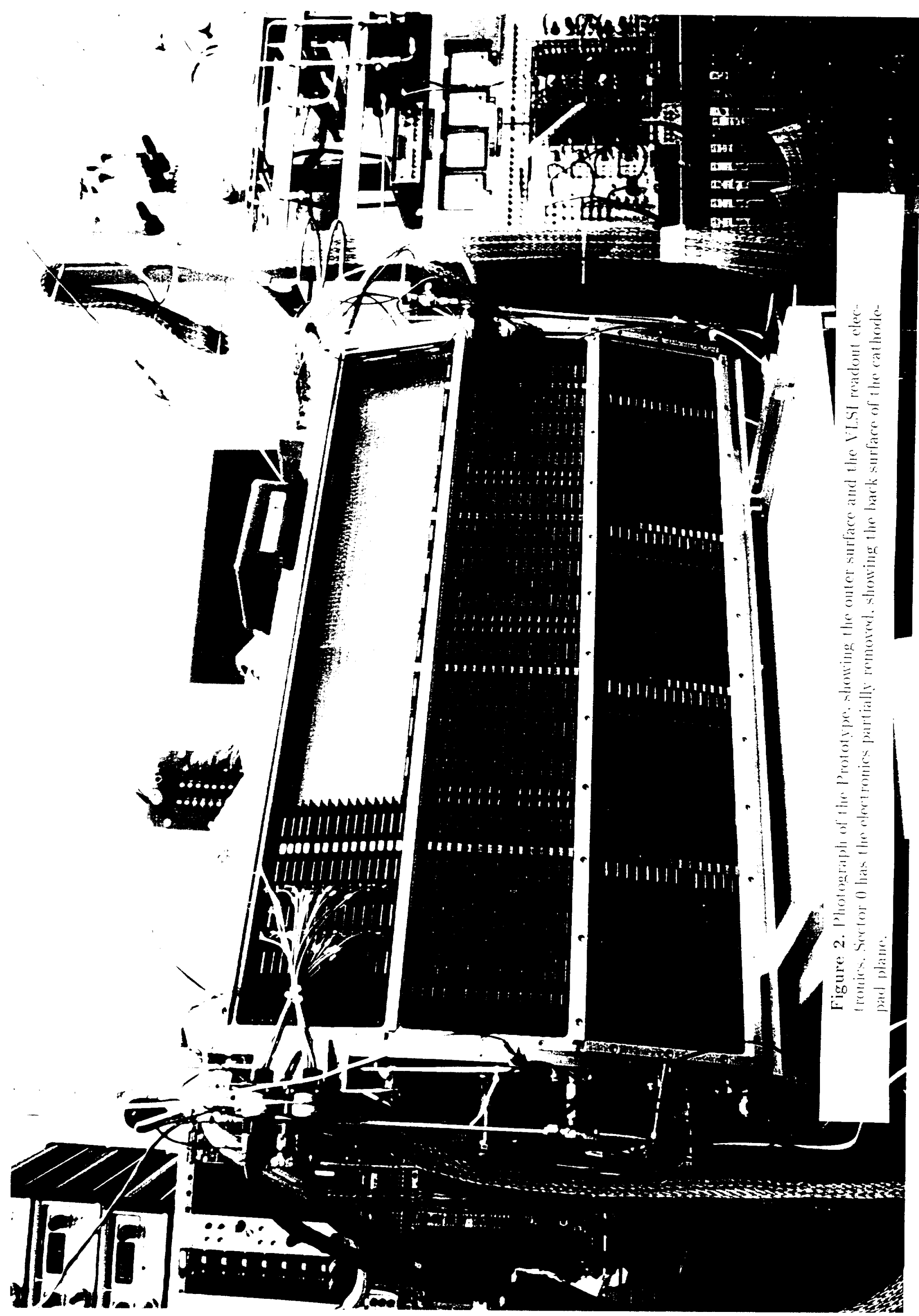



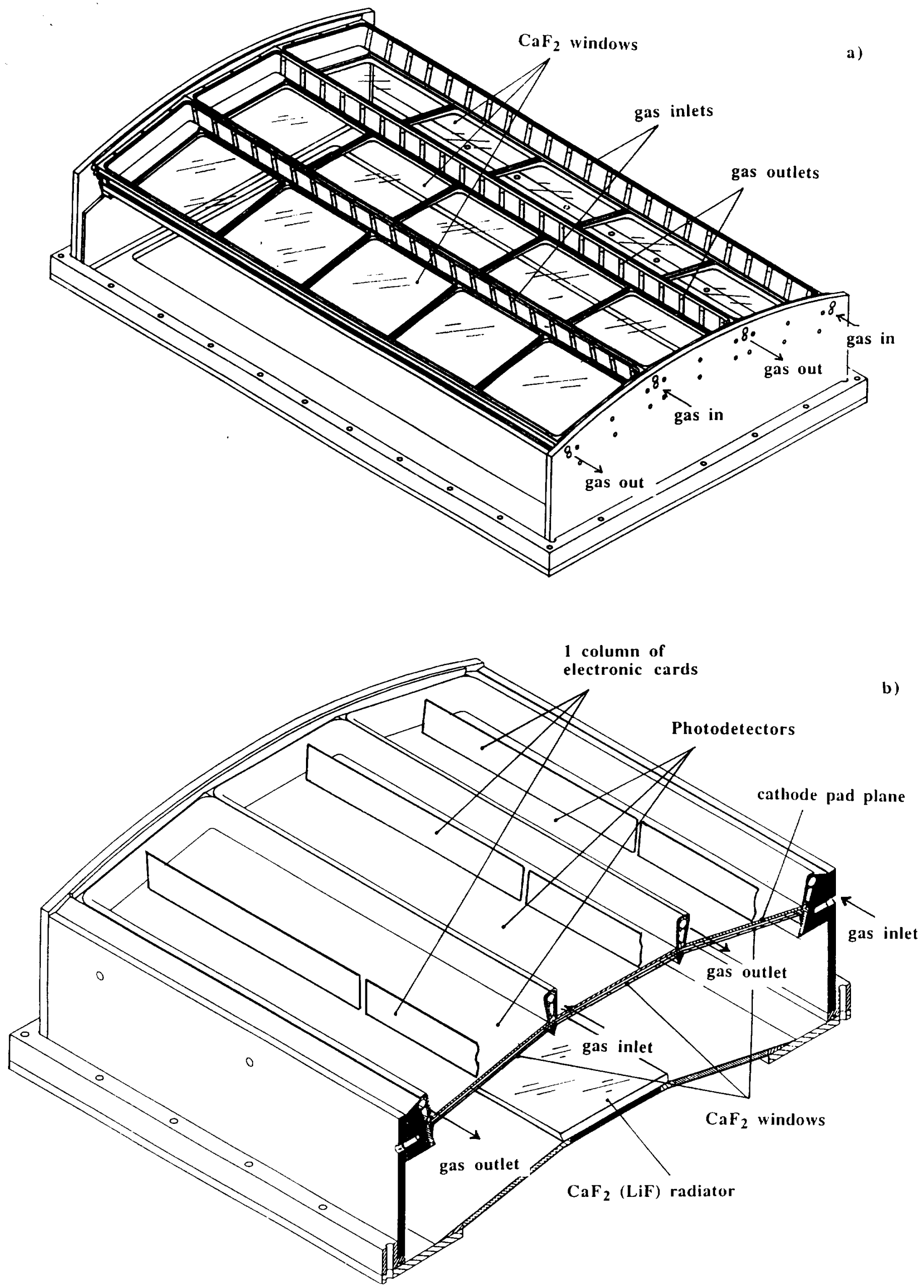

Figure 1. The mechanical structure of the full-scale barrel Fast-RICH Prototype. (a) The three sectors and the hollow structures above the $\mathrm{CaF}_{2}$ windows which receive the photodetectors. $(b)$ A transverse cut of the Prototype, showing the radiators and photon detectors in place, as well as the principle of the mounting of the VLSI readout electronics. 


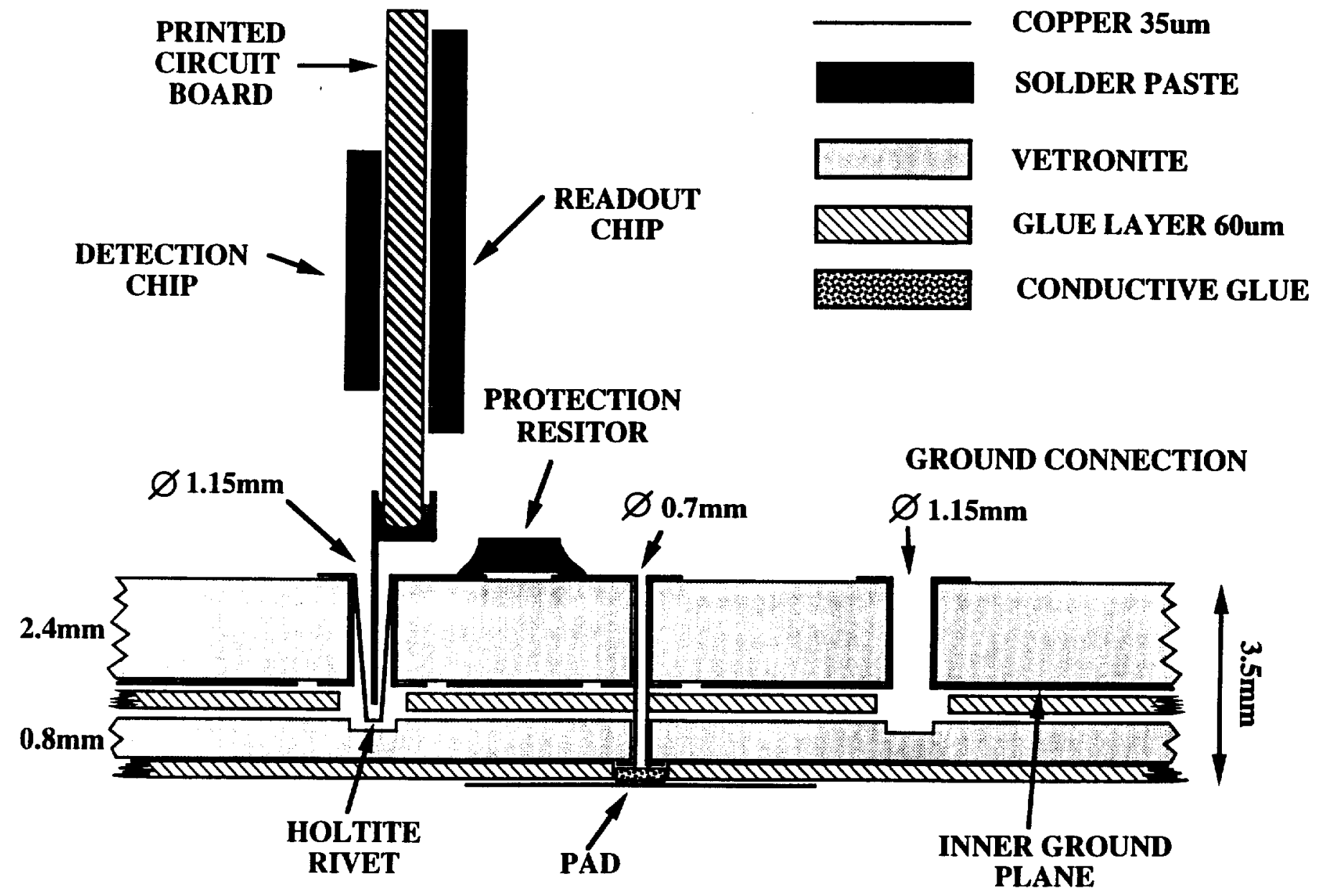

Figure 3. Detailed schematic of the design and the fabrication of the cathode-pad plane, with one $\mathrm{PCB}$ indicating the analog (detection) and digital (readout) electonics. 


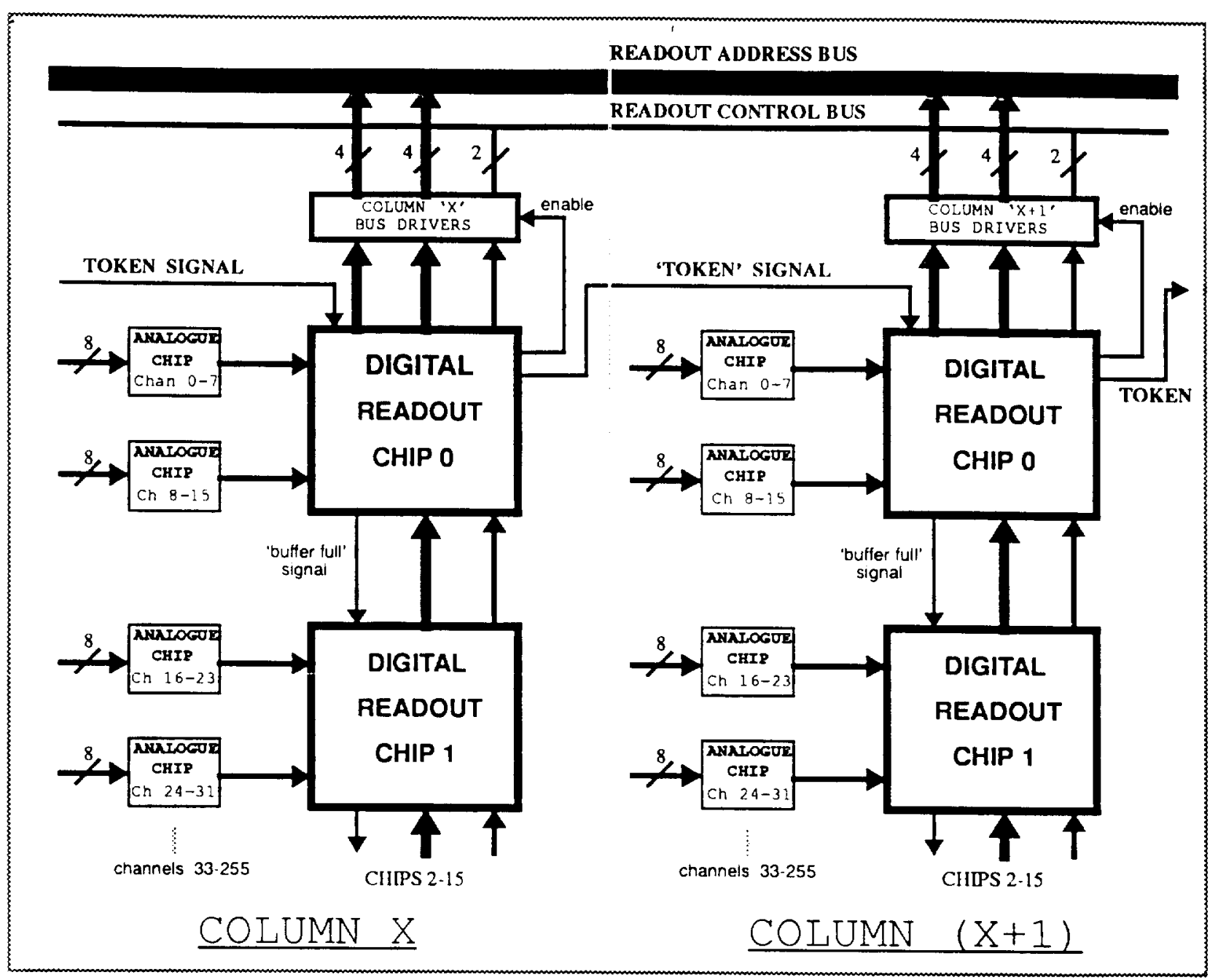

Figure 4. The chip-set with two analog bipolar chips per digital CMOS chip, shown for two columns. 


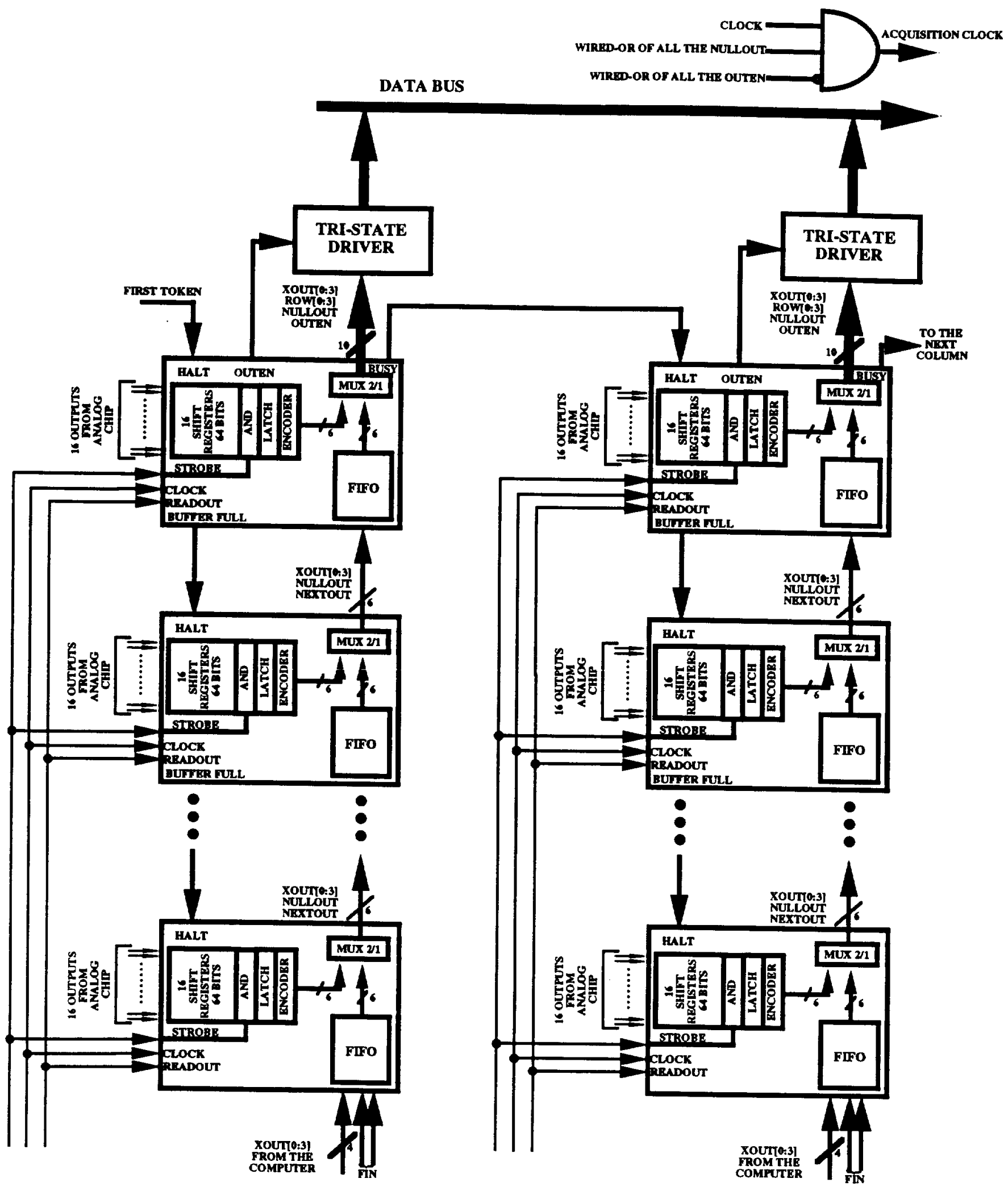

Figure 5. VLSI readout electronics pipeline, showing the daisy-chain, shift-register delay, and FIFO. 


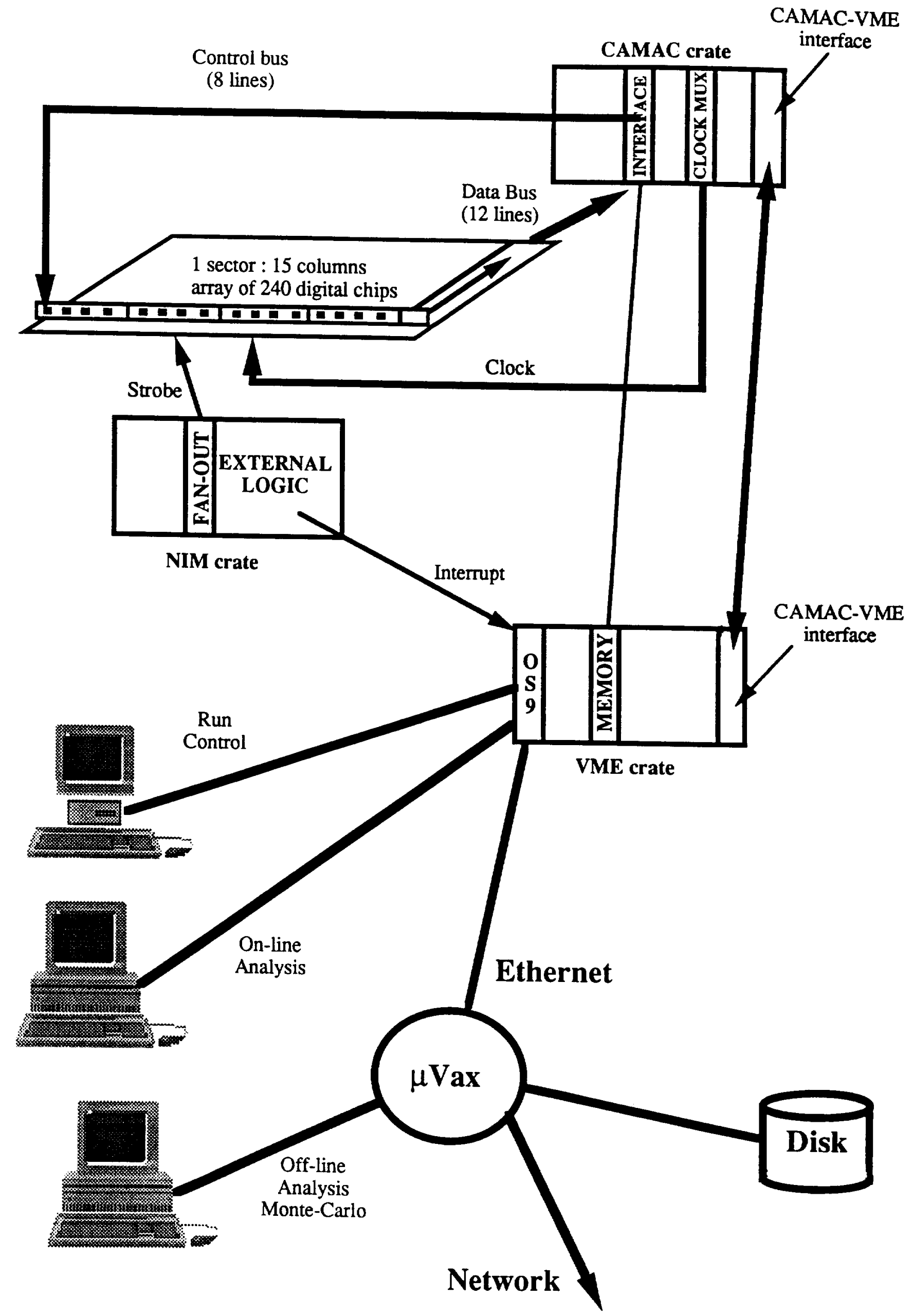

Figure 6. A schematic overview of the data acquisition system. The readout clock and strobe (trigger-generated latch) signal are generated externally to the on-board electronics. 

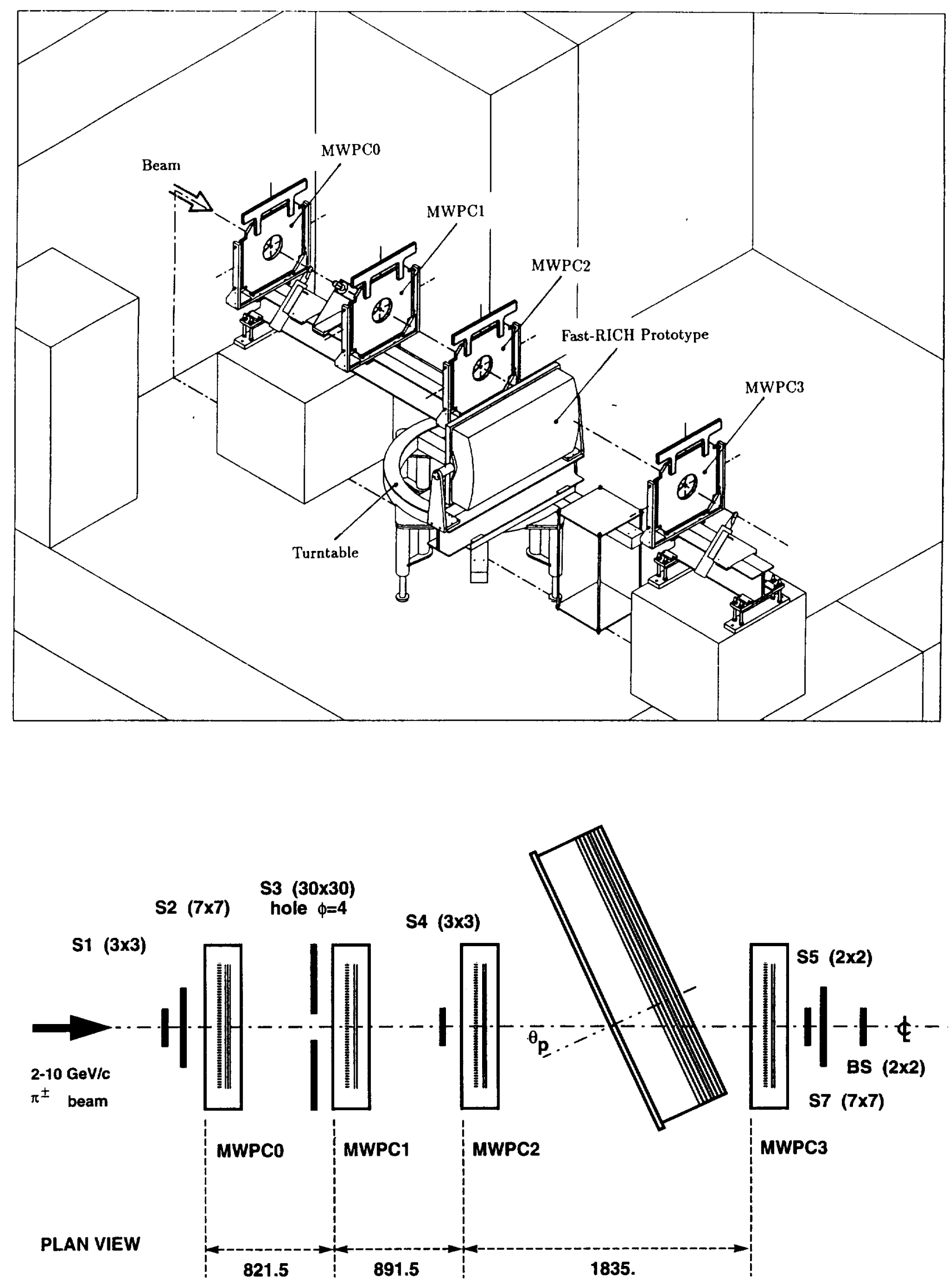

Figure 7. The beam test setup, showing the Fast-RICH Prototype, scintillation counters $S_{1}-$ $S_{6}$, wire chambers MWPC0-MWPC3, mechanical design of the mountings, support structure, turntable, and CERN PS T9 beam line. (a) EUCLID CAD isometric view. (b) Schematic layout in plan view (all dimensions in $\mathrm{mm}$ ). 

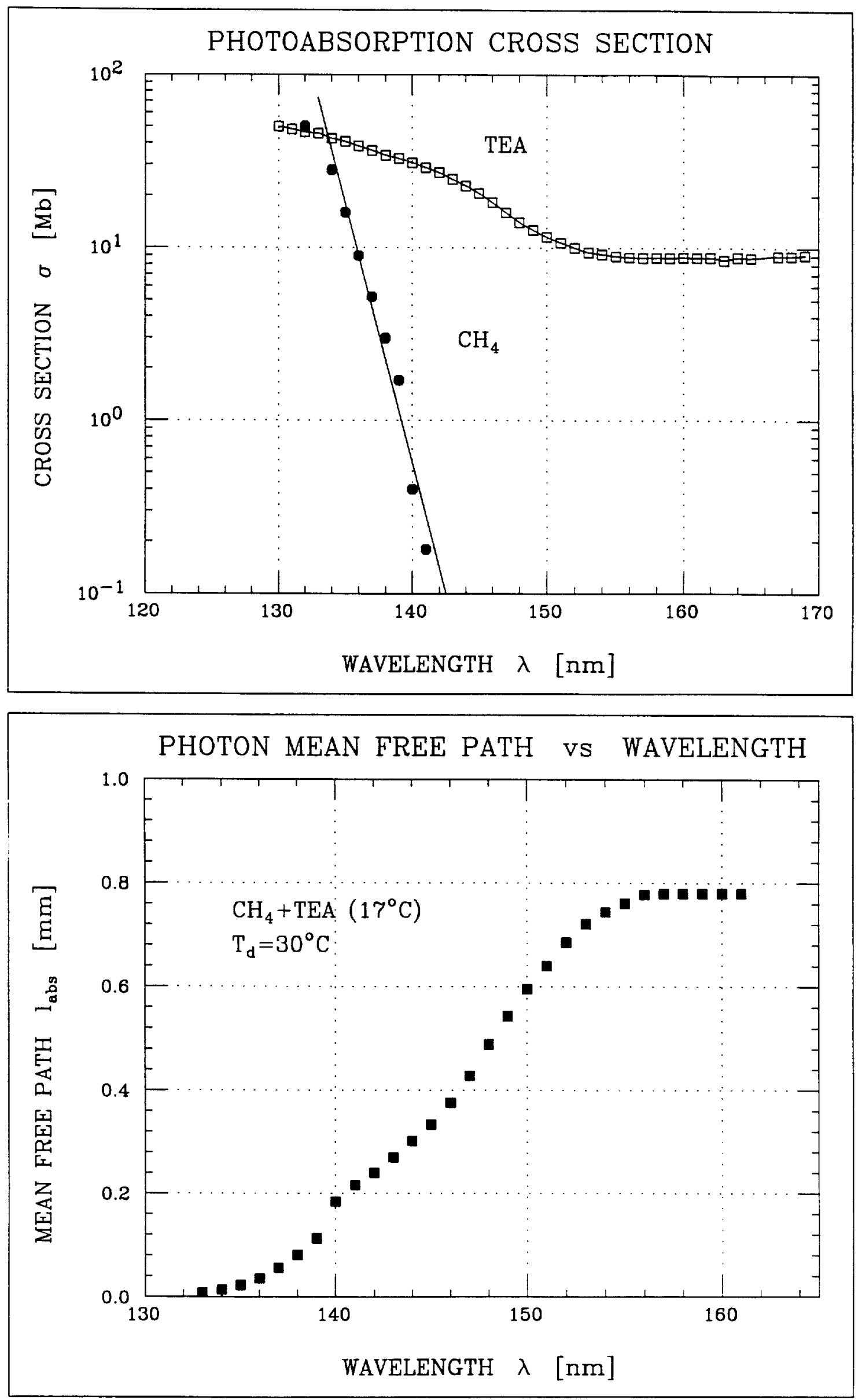

Figure 8. (a) The photoabsorption cross sections of TEA and $\mathrm{CH}_{4}$. (b) The absorption length $\ell_{\text {abs }}$ for the $\mathrm{CH}_{4}+$ TEA gas mixture as a function of wavelength, calculated by Equation (3) under beam test operating conditions $\left(T_{b}=17^{\circ} \mathrm{C}, T_{\text {det }}=30^{\circ} \mathrm{C}\right)$. At $150 \mathrm{~nm}, \ell_{\text {abs }}=0.6 \mathrm{~mm}$. 


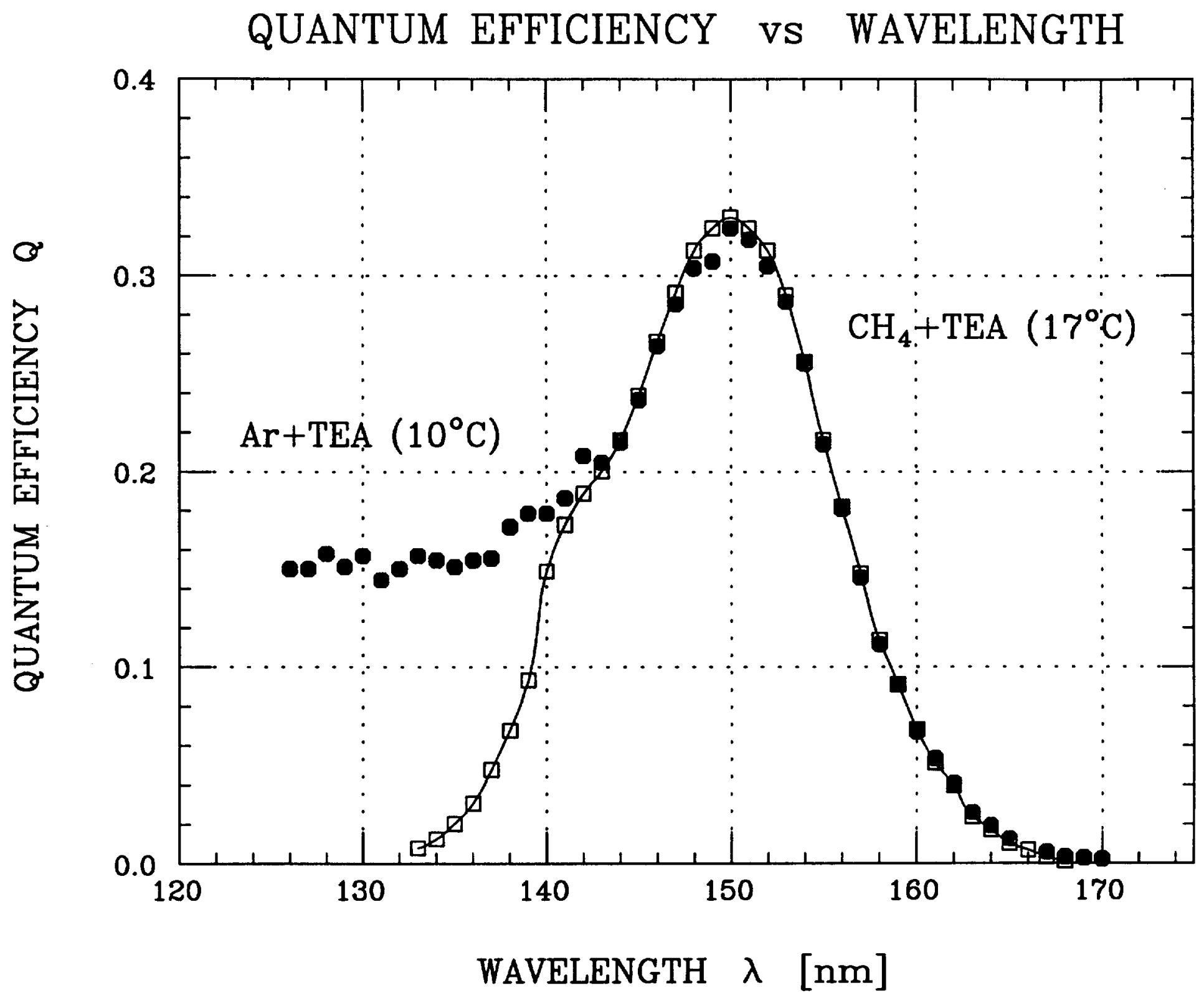

Figure 9. The quantum efficiency measurements of: $(\bullet)$ TEA in $\operatorname{Ar}[2,19]$; and ( $\square$ ) TEA in $\mathrm{CH}_{4}$, under beam test conditions. 

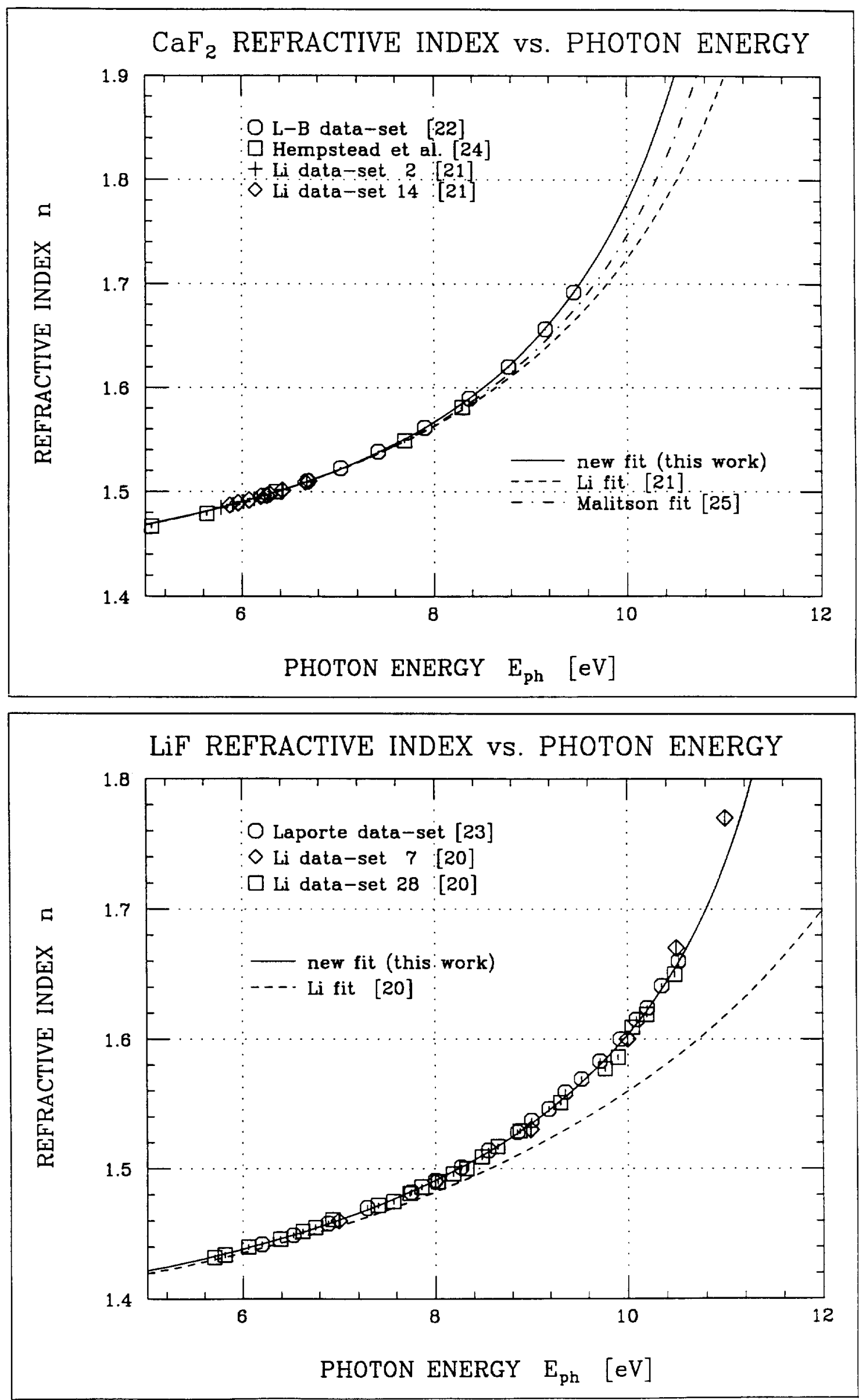

Figure 10. The refractive indices of the radiators in the UV region: (a) $\mathrm{CaF}_{2}$. (b) $\mathrm{LiF}$. Data used for our fits are shown along with previous Sellmeier fits (see text and Table II). Error bars are as indicated. (For clarity, some of the $\mathrm{CaF}_{2}$ data points with larger error-bars have been suppressed.) 

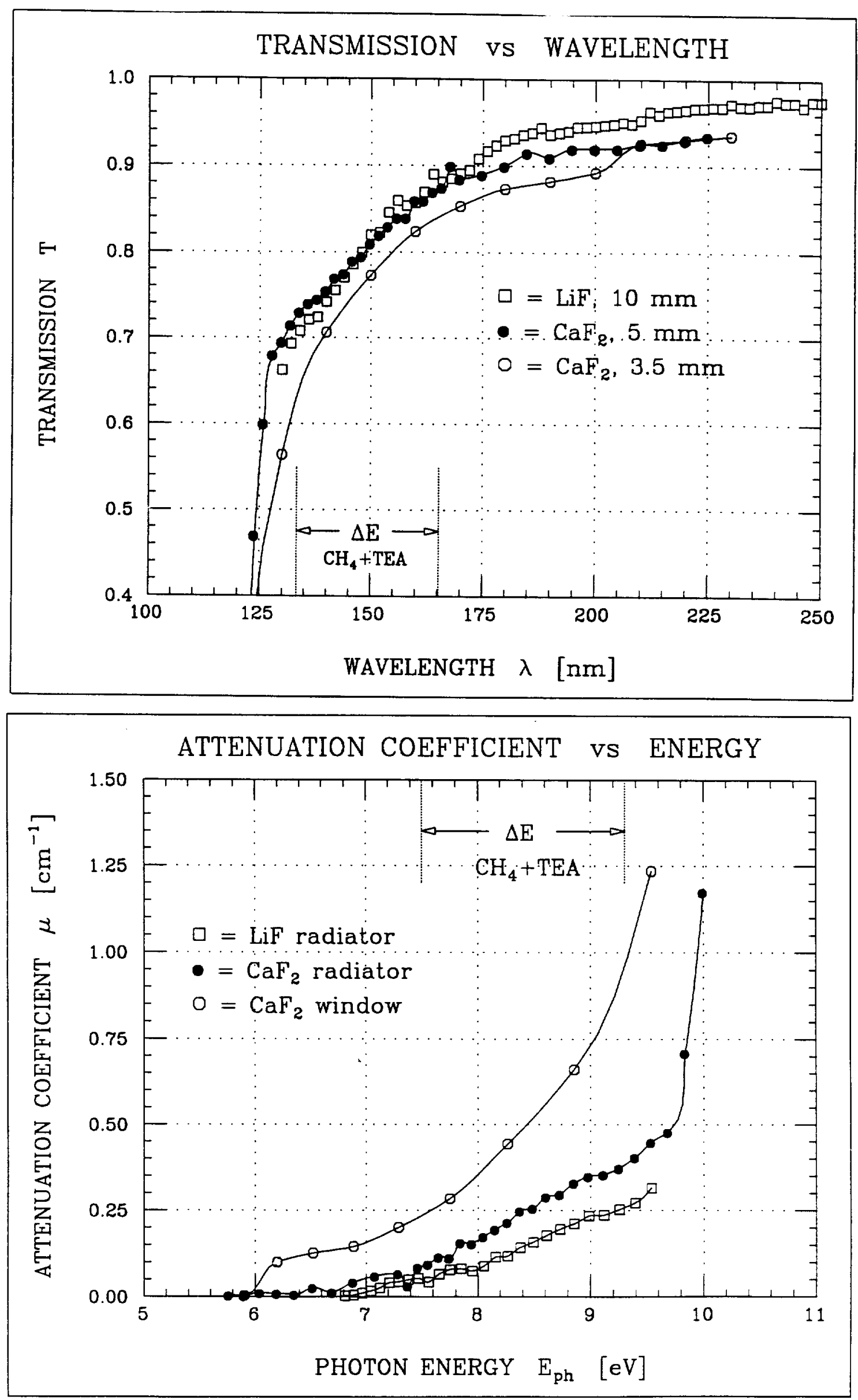

Figure 11. (a) Transmission measurements at normal incidence of a $10 \mathrm{~mm}$ thick LiF radiator, a $5 \mathrm{~mm} \mathrm{CaF}$ radiator, and a $3.5 \mathrm{~mm}$ thick $\mathrm{CaF}_{2}$ window, as a function of wavelength. (b) The attenuation coefficient $\mu$ of the same samples, extracted from the transmission data. 


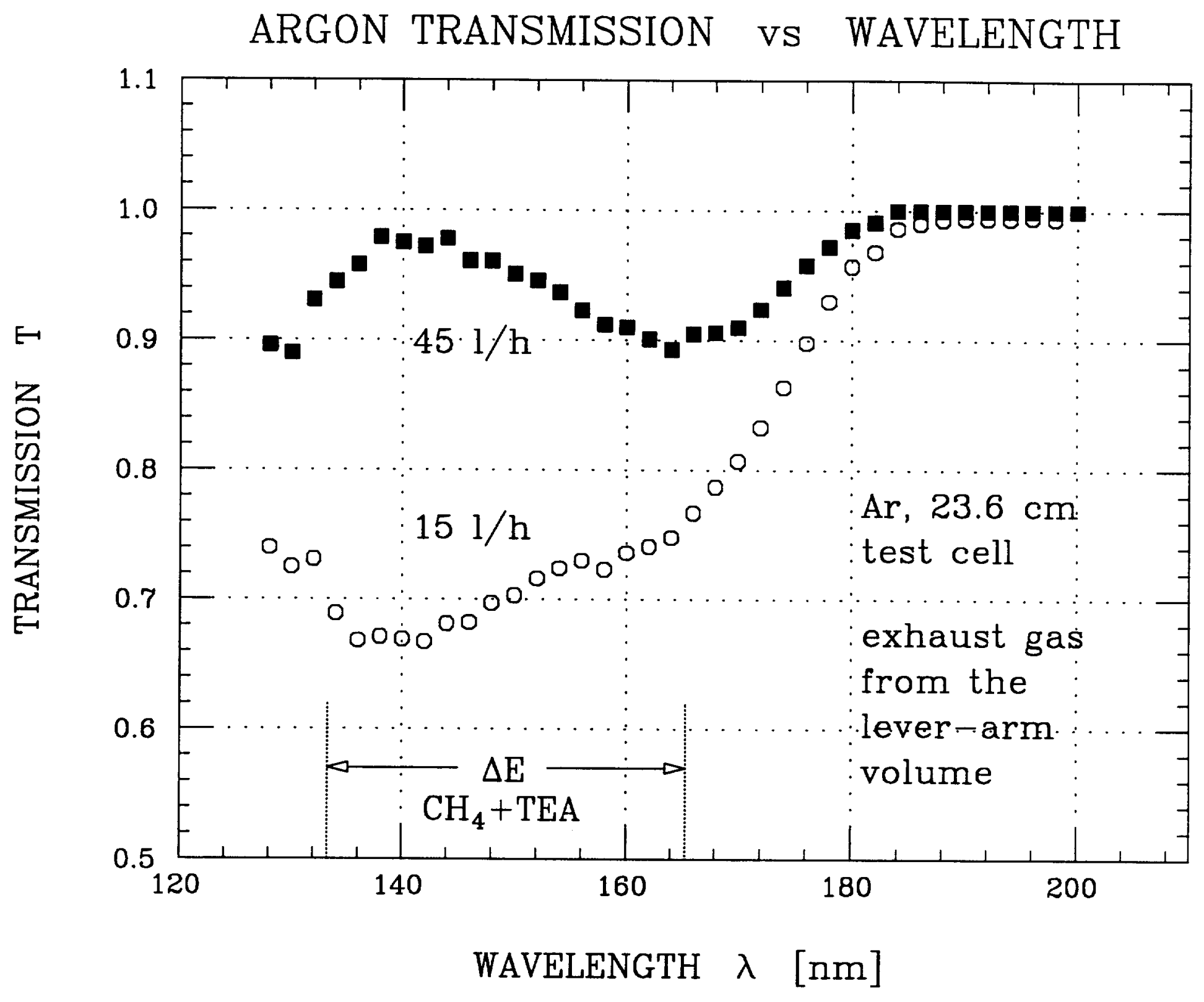

Figure 12. UV transparency of the lever-arm volume as a function of photon wavelength under different gas flow conditions ( 45 and $15 \ell / h$ ). Note the absorption due to $\mathrm{H}_{2} \mathrm{O}$ centered at $\sim 165$ $\mathrm{nm}$, and $\mathrm{O}_{2}$ at $\sim 140 \mathrm{~nm}$. 

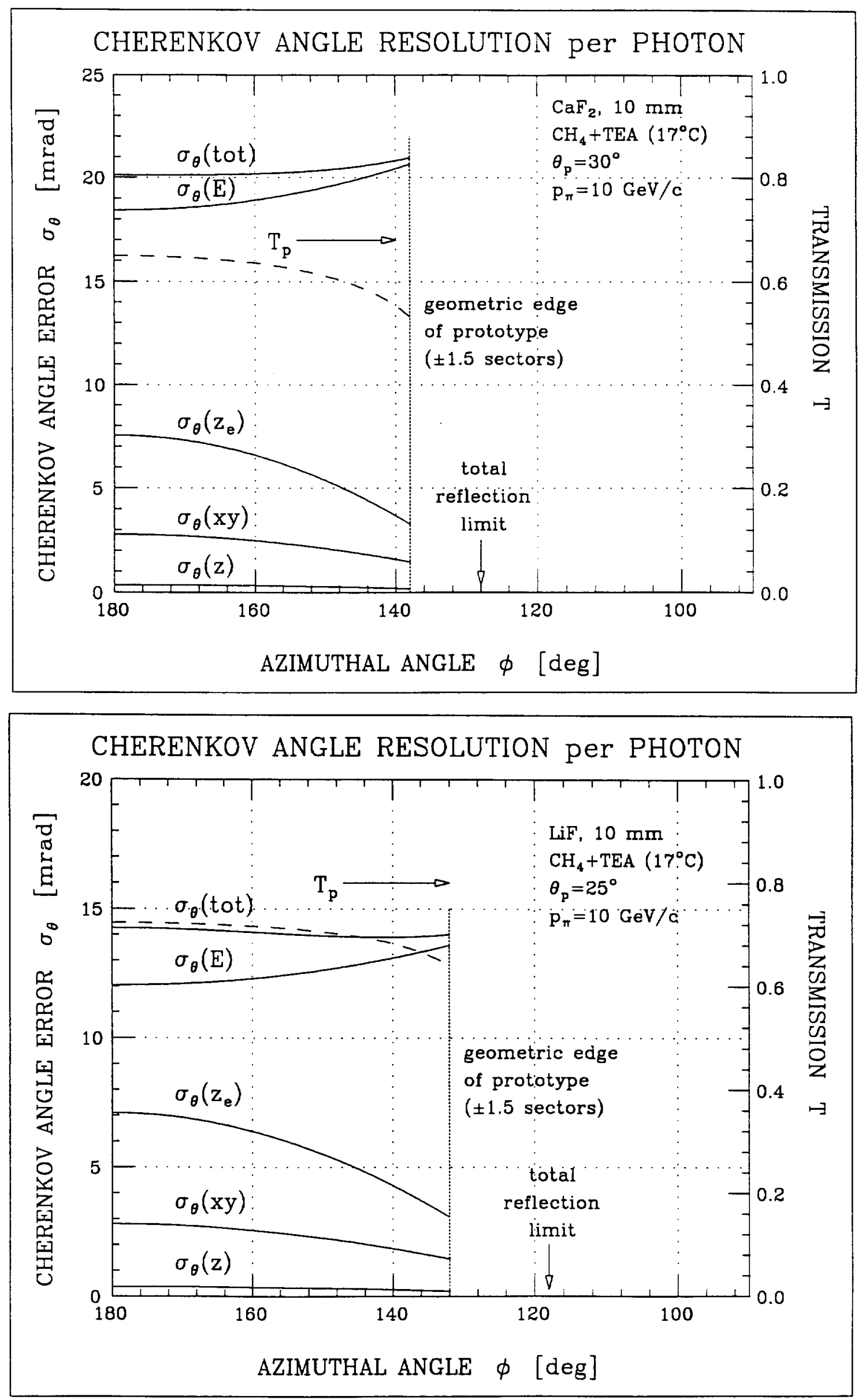

Figure 13. Analytical calculation of the various contributions to the single-photon Cherenkov polar angle $\theta$ resolution as a function of the Cherenkov azimuthal angle $\phi$. Calculations made under conditions identical with the experimental data, for (a) $\mathrm{CaF}_{2}$ at $\theta_{p}=30^{\circ}$, and (b) $\mathrm{LiF}$ at $\theta_{p}=25^{\circ}$. The total internal reflection transmission limit is noted. 
(a) $\mathrm{CaF}_{2}-\Theta_{\mathrm{p}}=30^{\circ}-$ MONTE CARLO (4000 events)

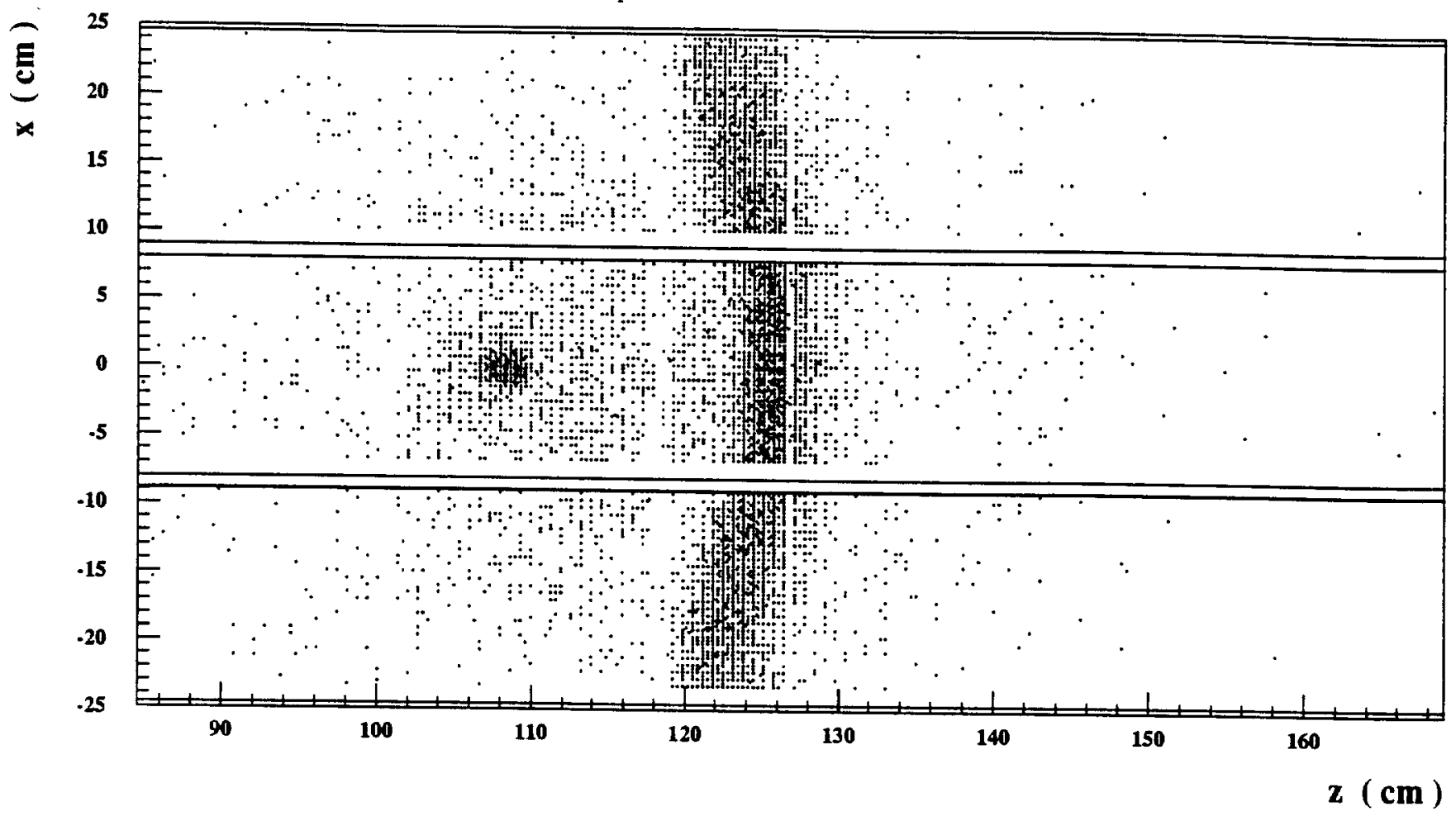

(b) $\operatorname{LiF}-\Theta_{p}=25^{\circ}-$ MONTE CARLO (5000 events)

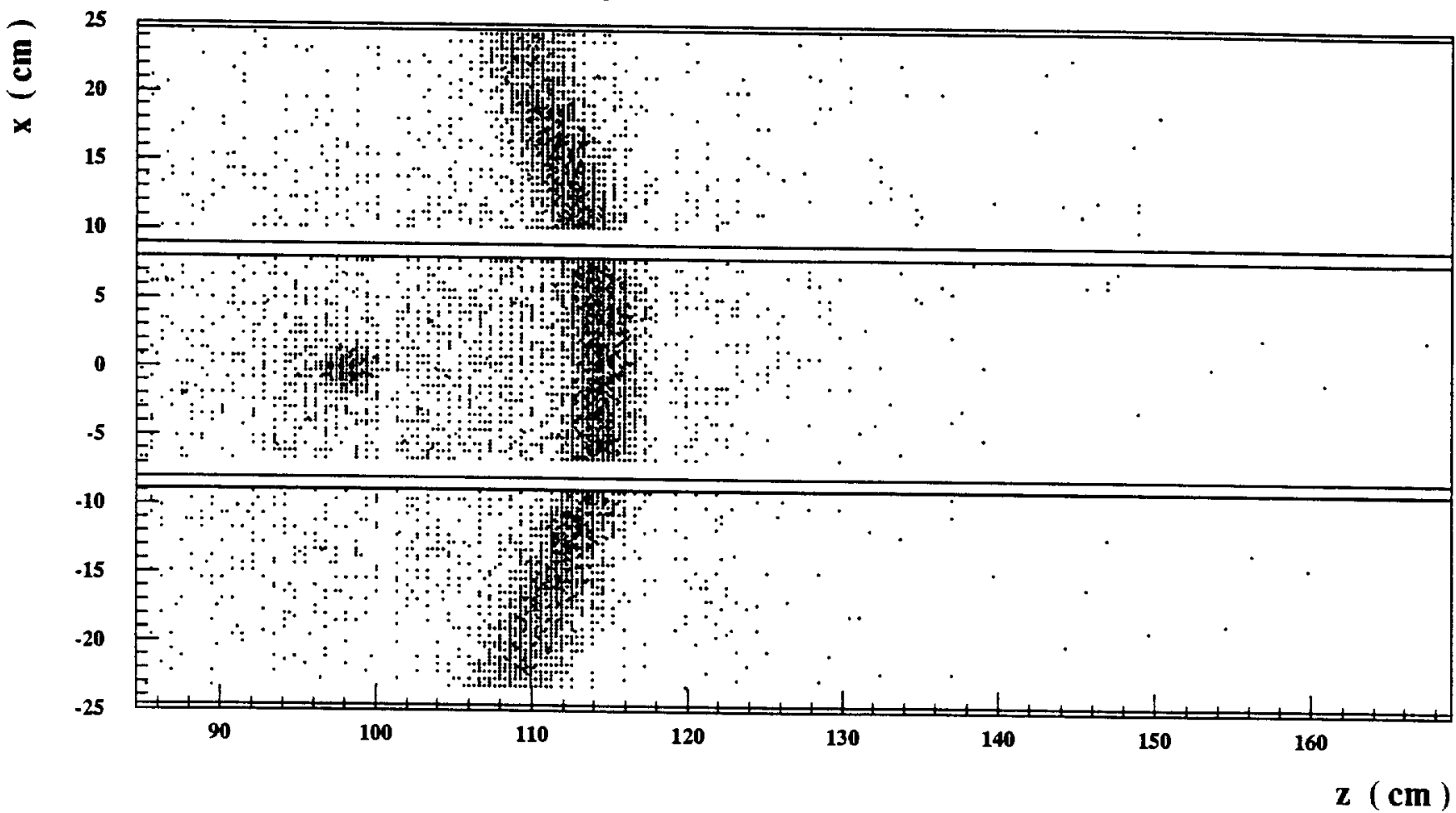

Figure 14. Cumulation of Monte Carlo images generated under experimental conditions, for (a) $4000 \mathrm{CaF}_{2}$ events at $\theta_{p}=30^{\circ}$, (b) $5000 \mathrm{LiF}$ events at $\theta_{p}=25^{\circ}$. 
(a) $\mathrm{CaF}_{2}-\Theta_{\mathrm{p}}=30^{\circ}-$ MONTE CARLO (4000 events)
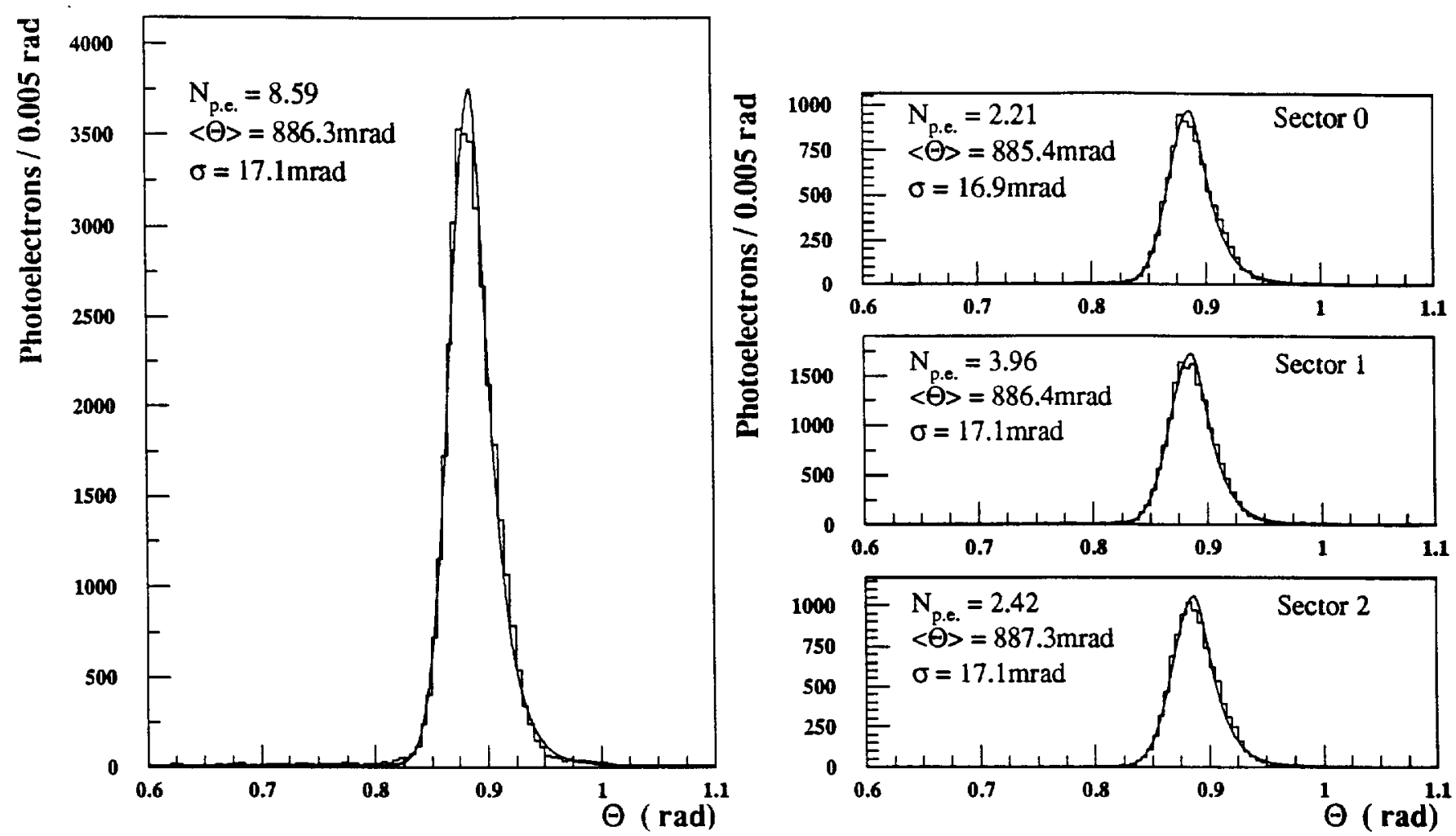

(b) LiF $-\Theta_{p}=25^{\circ}-$ MONTE CARLO (4000 events)
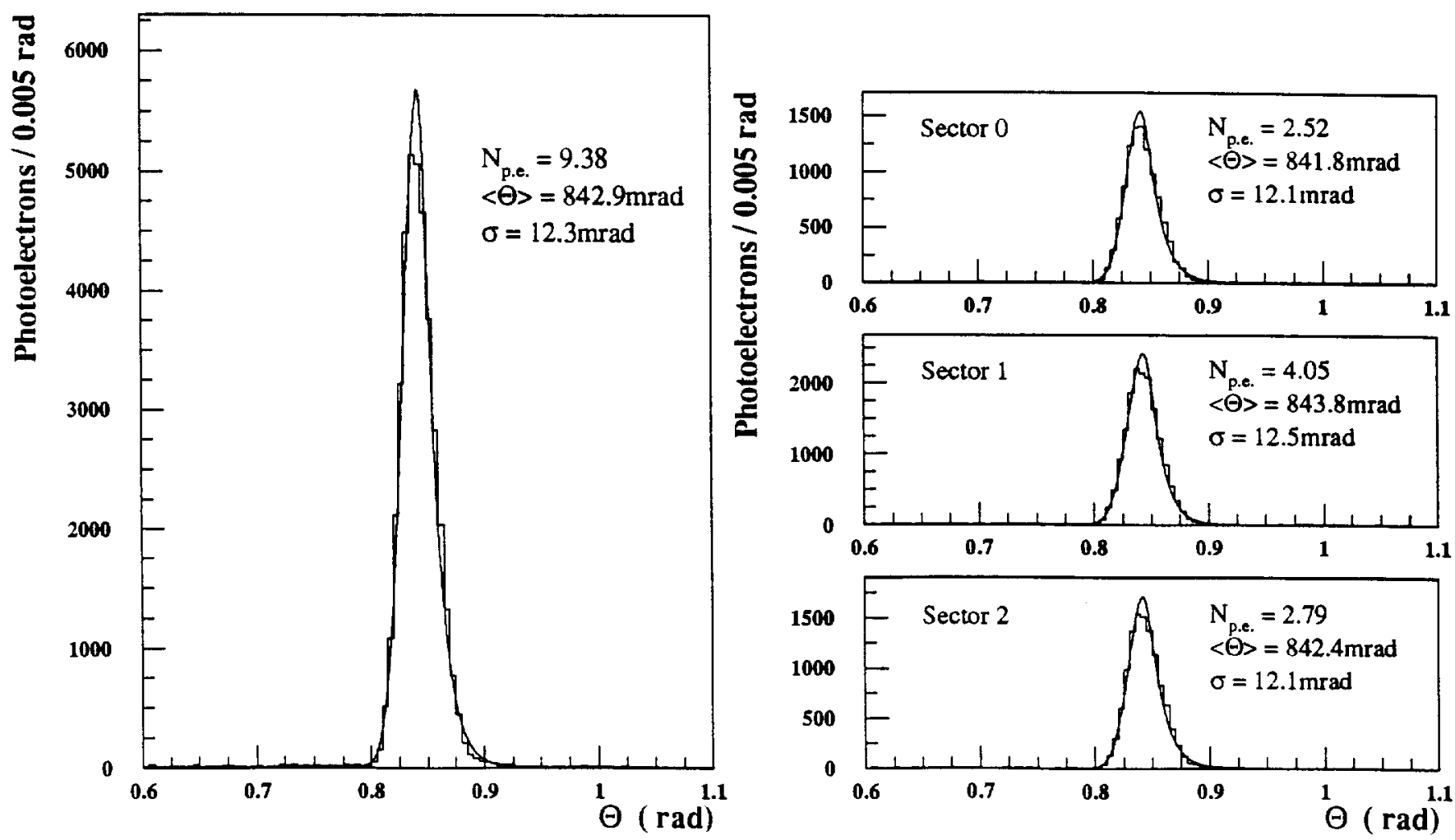

Figure 15. Monte Carlo single-photon Cherenkov angle distributions corresponding to events in Figure 14, for (a) $4000 \mathrm{CaF}_{2}$ events at $30^{\circ}$, (b) $5000 \mathrm{LiF}$ events at $25^{\circ}$. The LHS shows the total distribution, the RHS that for each of the individual sectors. (See text for an explanation of the fit parameters.) 


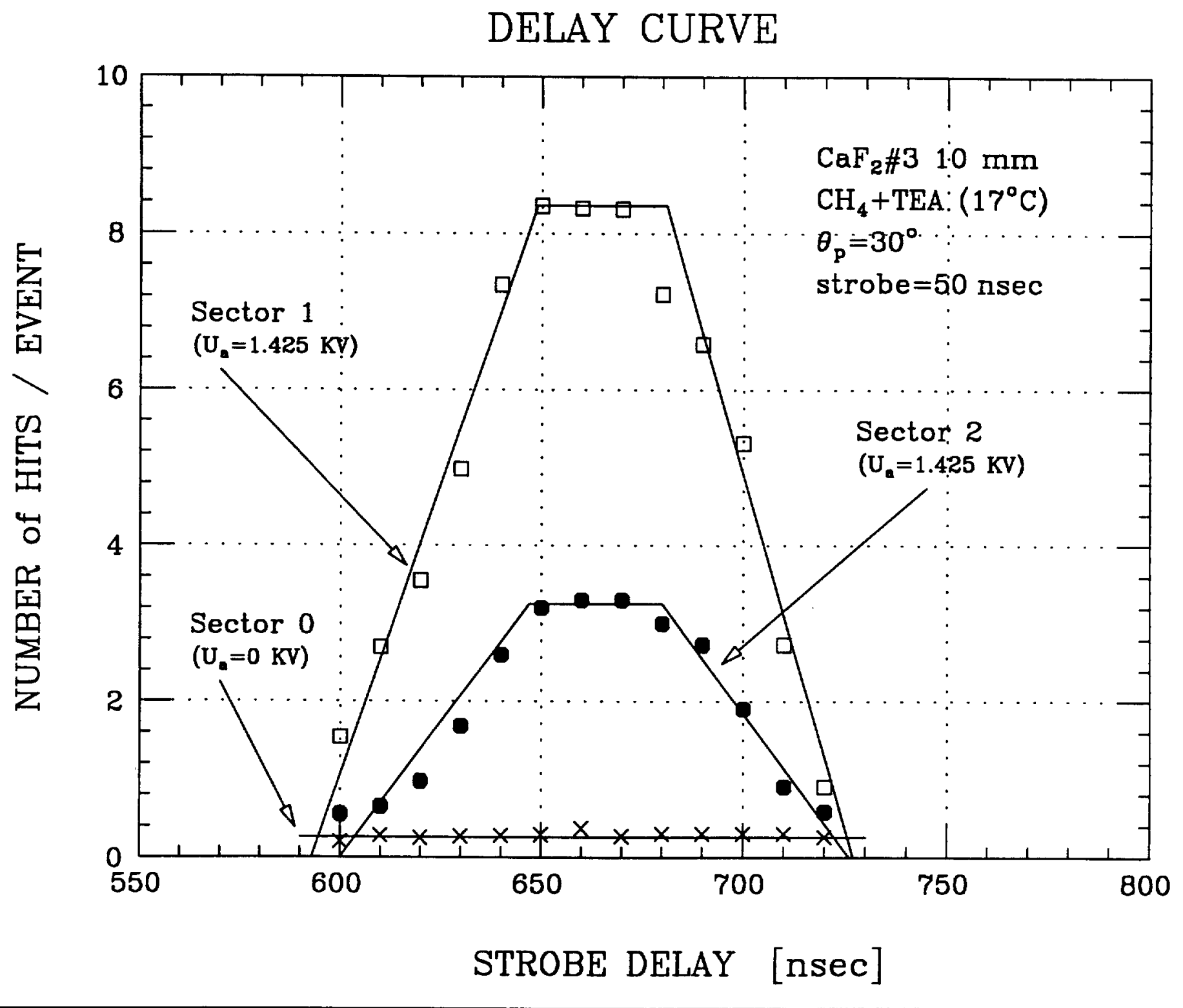

Figure 16. The delay curves obtained for a $\mathrm{CaF}_{2}$ radiator $\# 3$ at $\theta_{p}=30^{\circ}$ for all three sectors. 

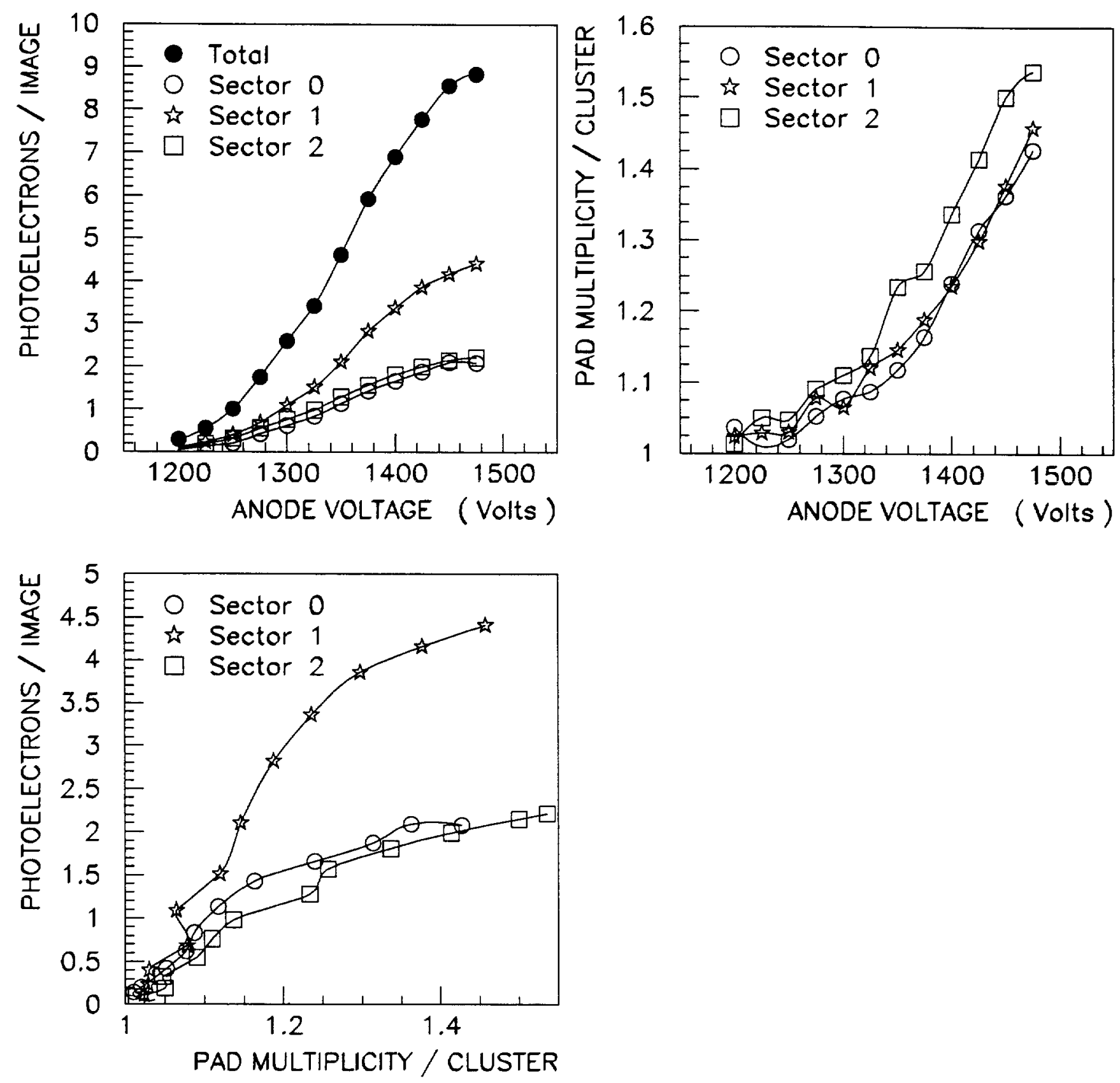

Figure 17. Correlation for voltage plateau correction. (a) The variation of the photoelectron yield (background subtracted) as a function of the anode wire voltage $U_{a}$. (b) The variation of the pad multiplicity per cluster vs. $U_{a}$. (c) The direct correlation between the photoelectron yield and the pad multiplicity per cluster. 


$$
\text { LiF }-\Theta_{p}=25^{\circ}-\text { DATA }
$$

(a) TEA

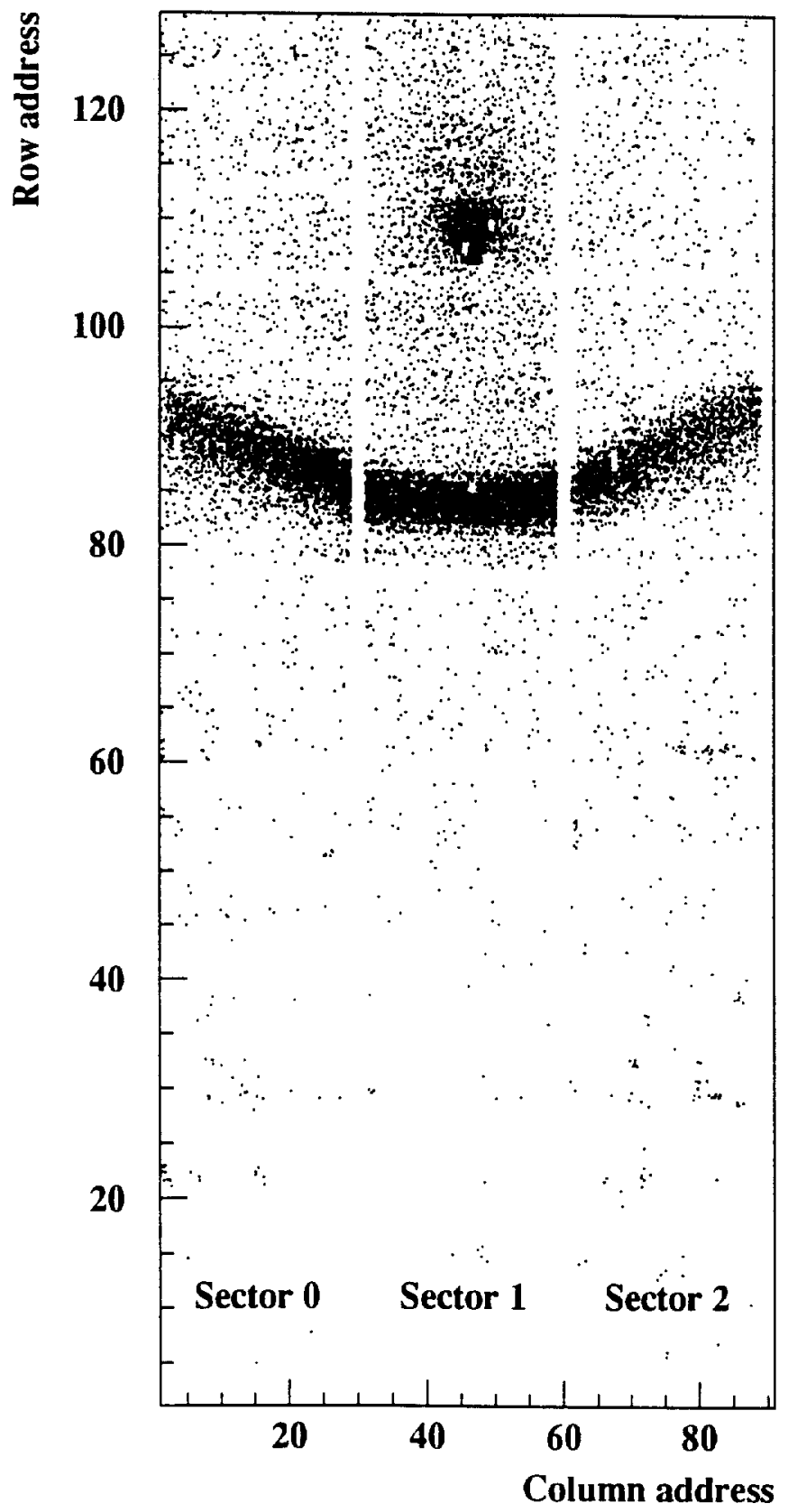

(b) no TEA

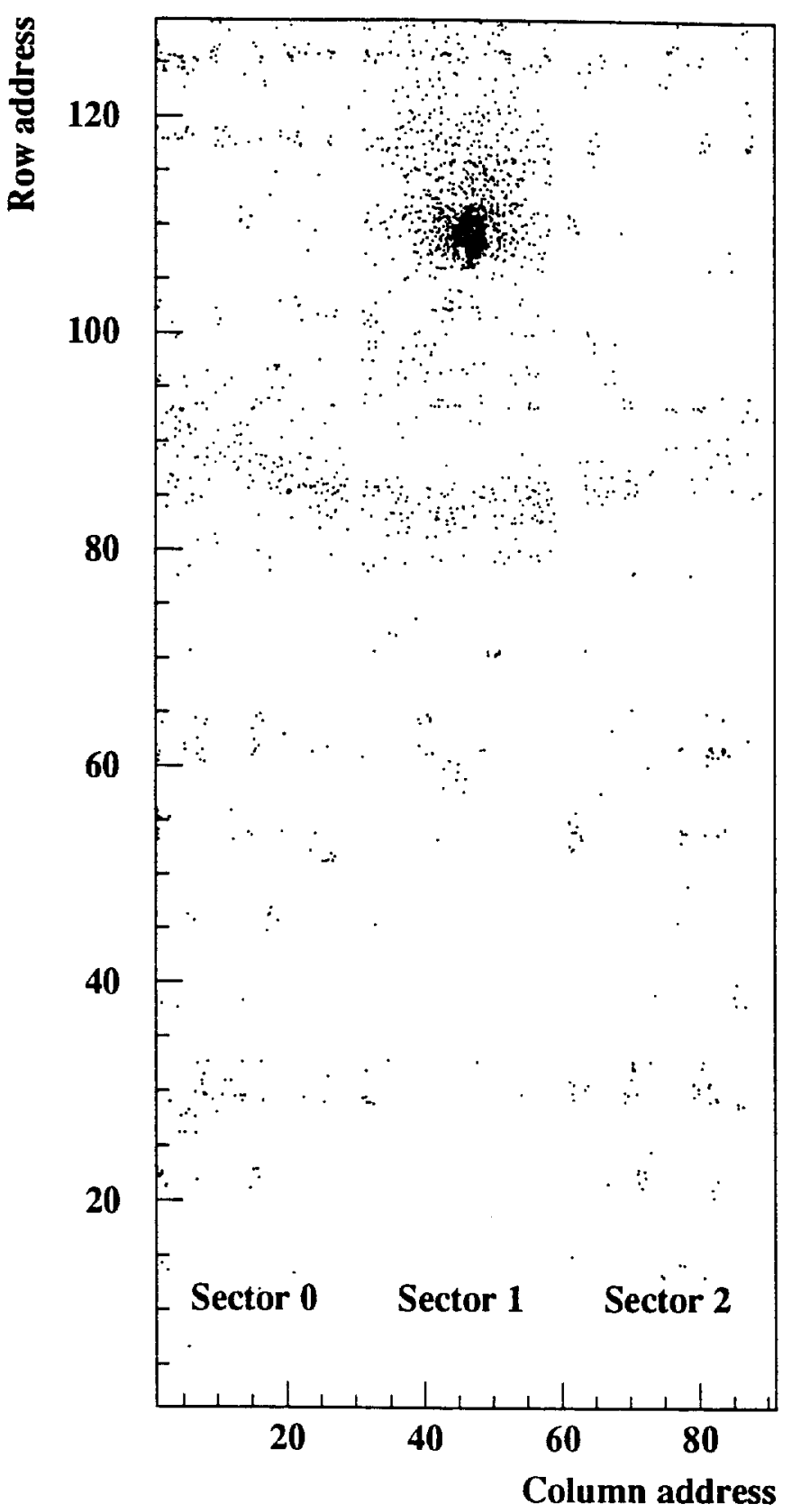

Figure 18. Raw events obtained in two LiF runs of 2000 events each at $\theta_{p}=25^{\circ}$, (a) with TEA in $\mathrm{CH}_{4}$, and (b) without TEA in $\mathrm{CH}_{4}$. Note the shadow of the detector structure, and the two dead channels in the image fiducial limits. Background levels are artificially enhanced visually by this scatter plot presentation. 


$$
\text { LiF - } \Theta_{p}=25^{\circ}-\text { DATA }
$$

(a) TEA

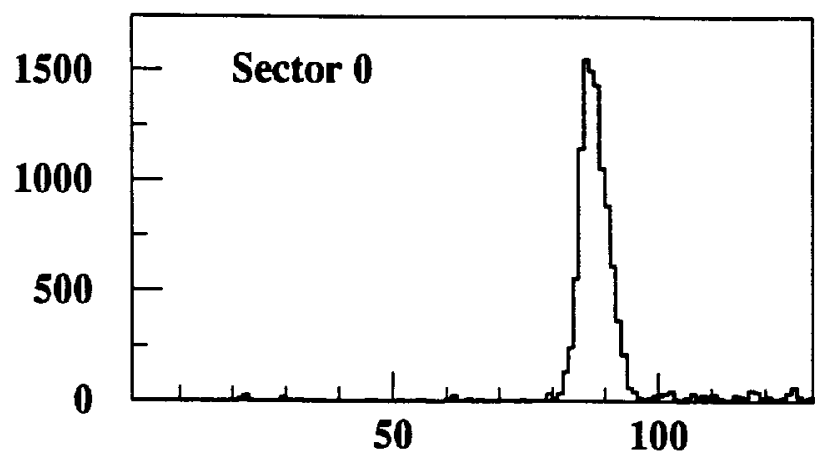

(b) no TEA

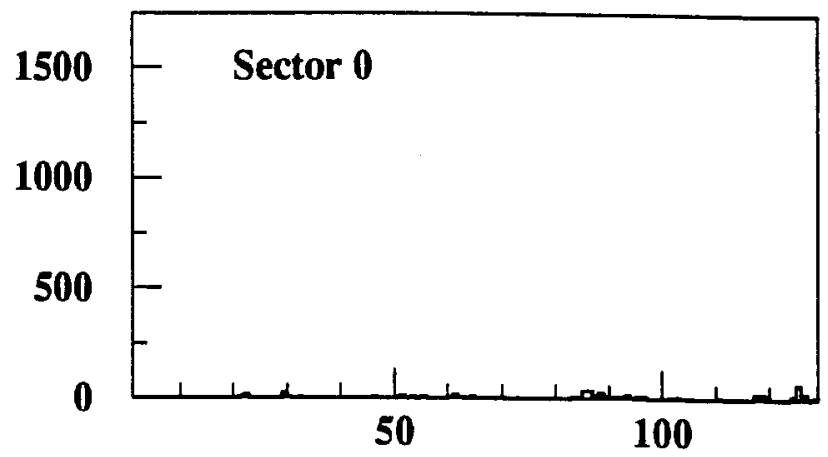

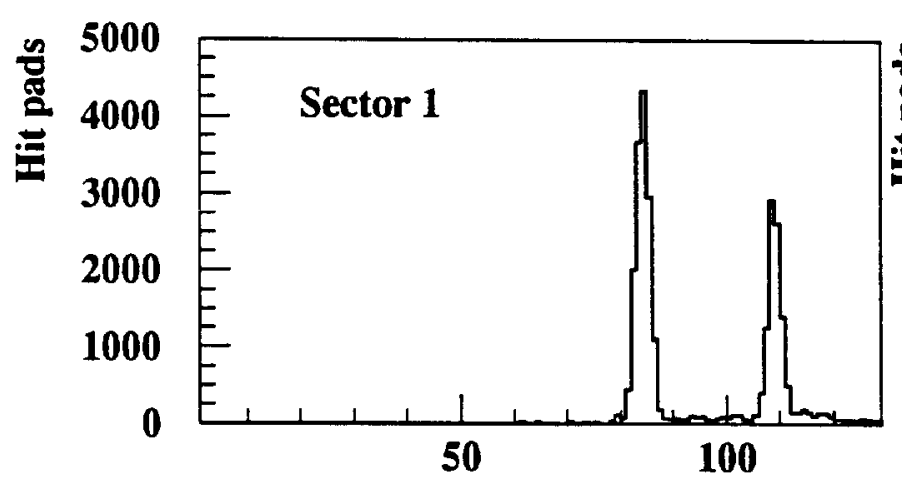
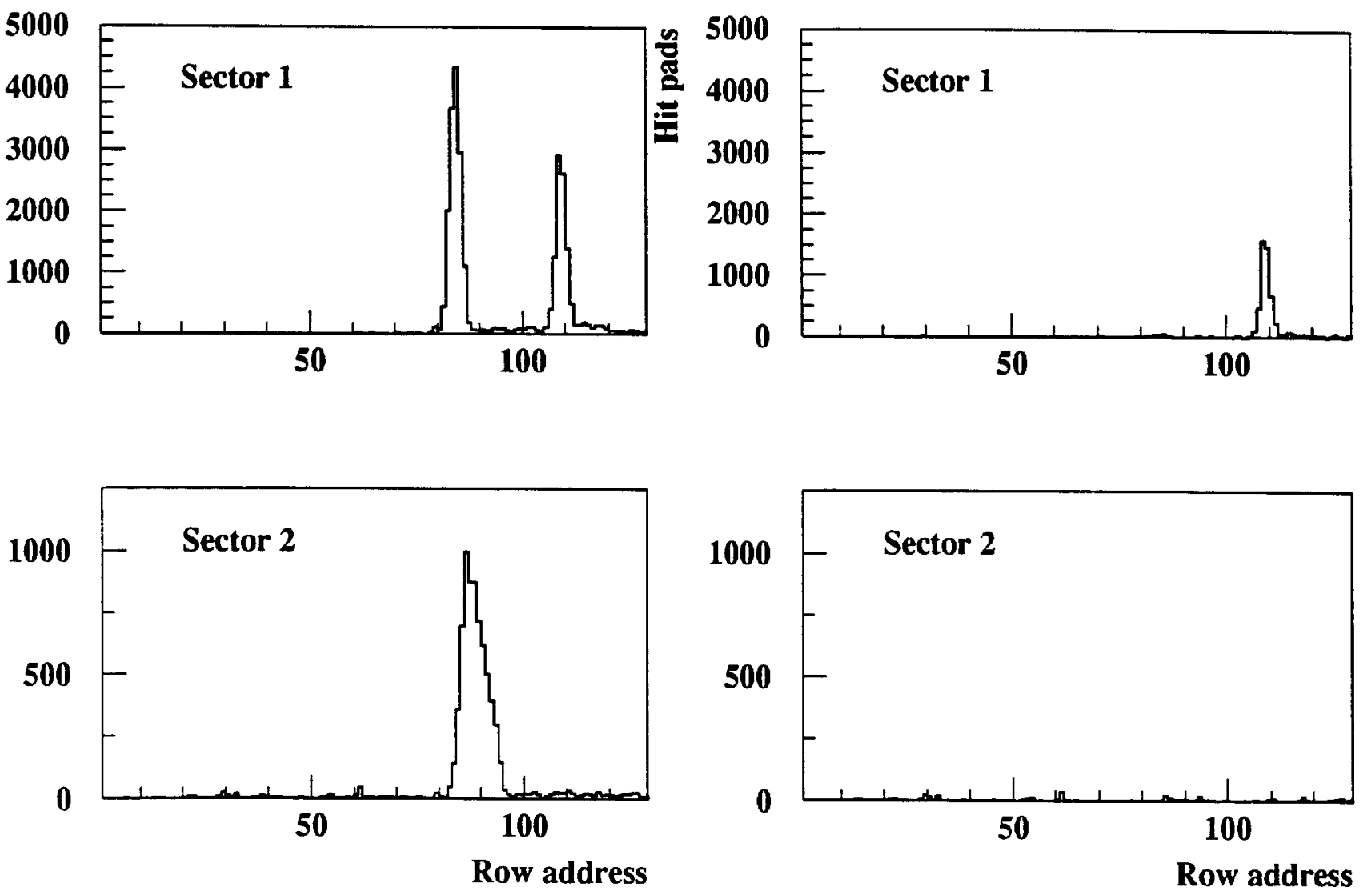

Figure 19. Projection of the LiF raw events of Figure 18, showing a profile of the hit rows, (a) with TEA in $\mathrm{CH}_{4}$, and (b) without TEA in $\mathrm{CH}_{4}$. The Cherenkov ring in projection and beam spot (in Sector 1) are evident; the background is clearly quite small. 


$$
\text { LiF }-\Theta_{p}=25^{\circ}-\text { DATA }
$$
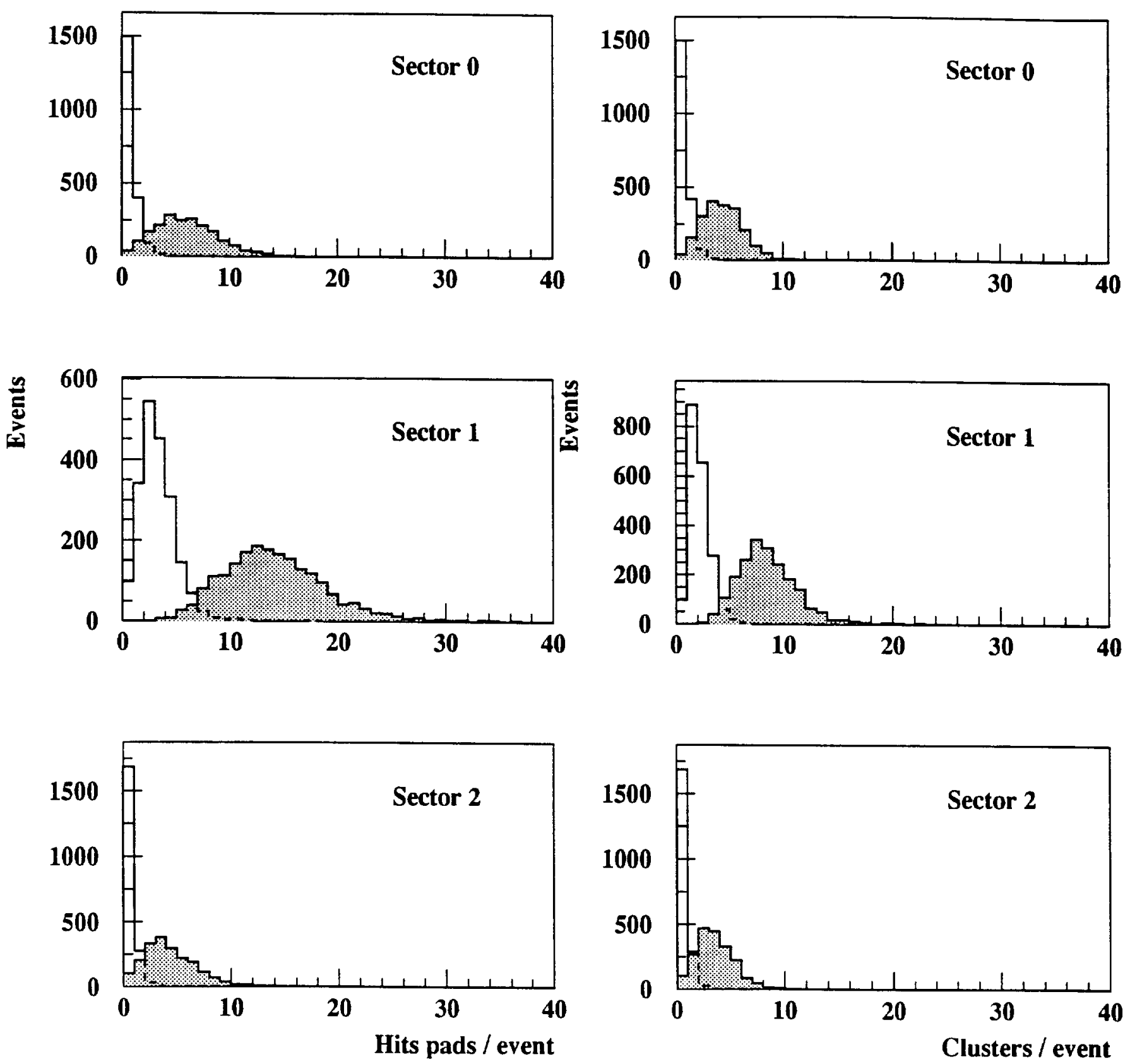

Figure 20. The raw data distributions (for each sector) of: (a) the number of hit pads per event, and (b) the number of clusters per event, without fiducial cuts. The shaded distributions are those with TEA, the unshaded without TEA. 

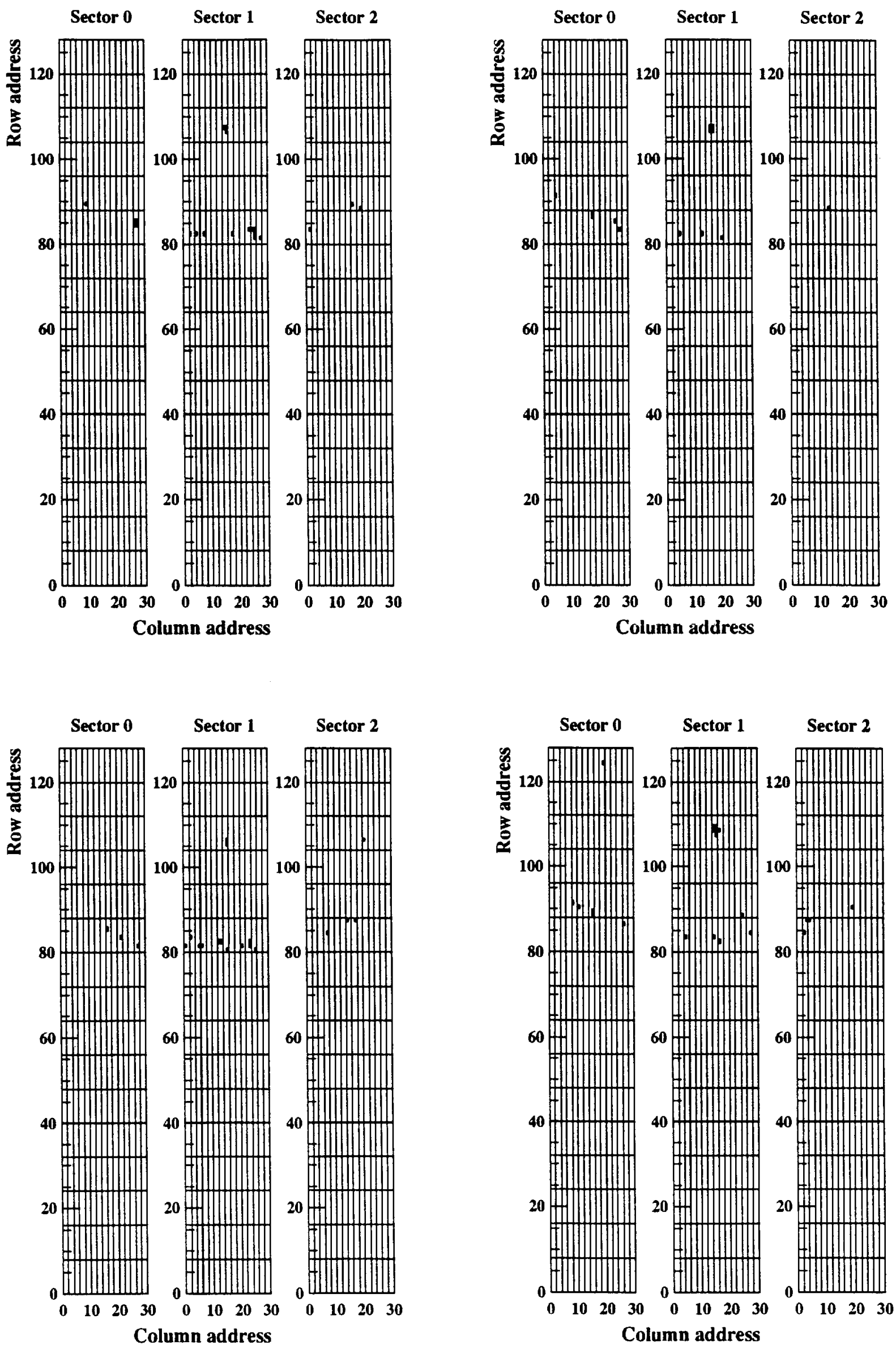

Figure 21. A sampling of individual raw events. A dark square is a hit pad; a rectangle represents $2 \times 8$ pads (i.e., a single digital chip). Note the number of hits per image, the clear beam spot, and the small amount of background. 


\section{LiF $-\Theta_{p}=25^{\circ}-$ DATA $(11805$ events $)$}

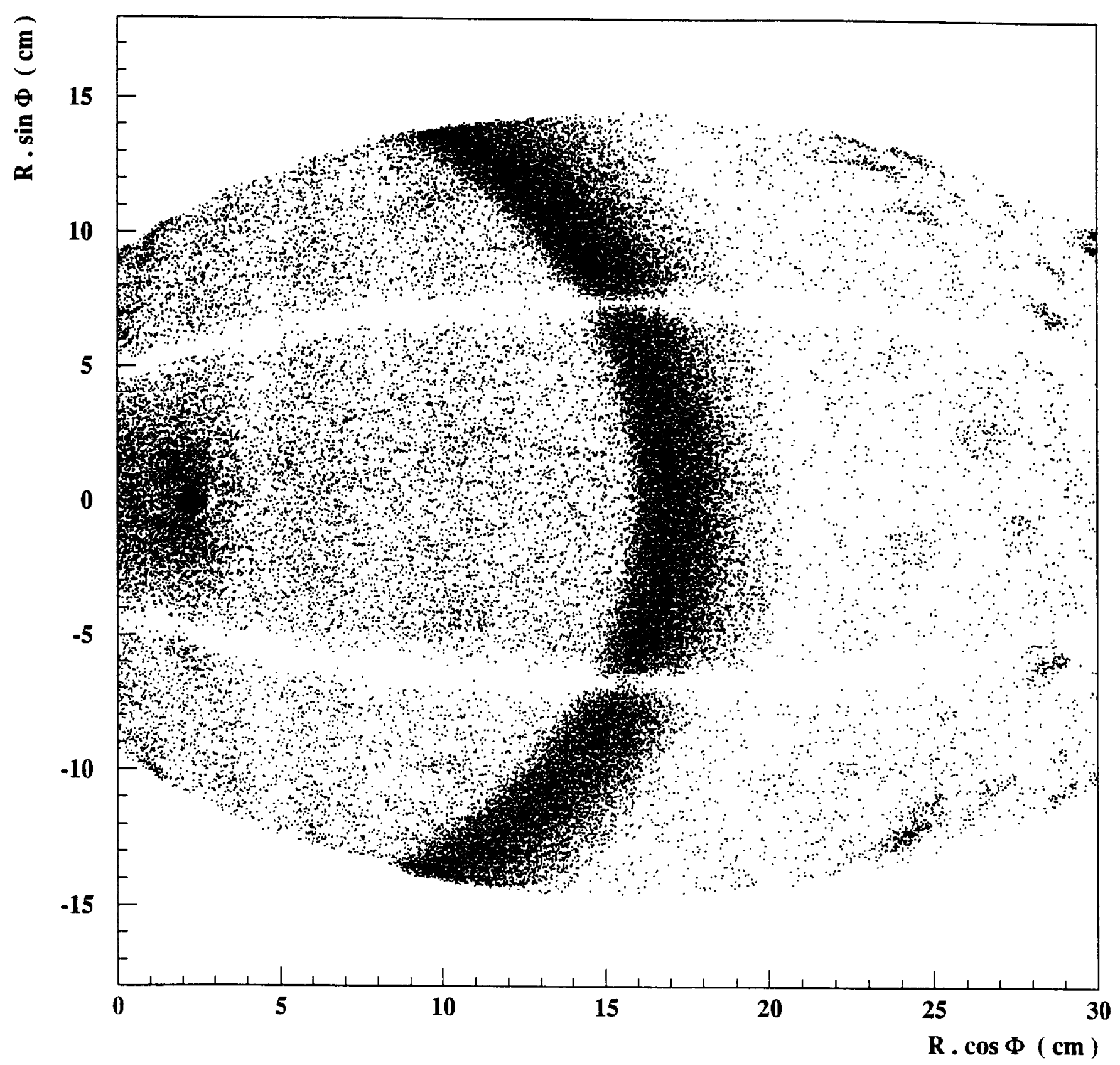

Figure 22. Cumulation of 11805 reconstructed images projected onto a plane perpendicular to the beam direction via the transformation of Equations (9). This is the full $\operatorname{LiF} \# 2 \theta_{p}=25^{\circ}$ data sample. 

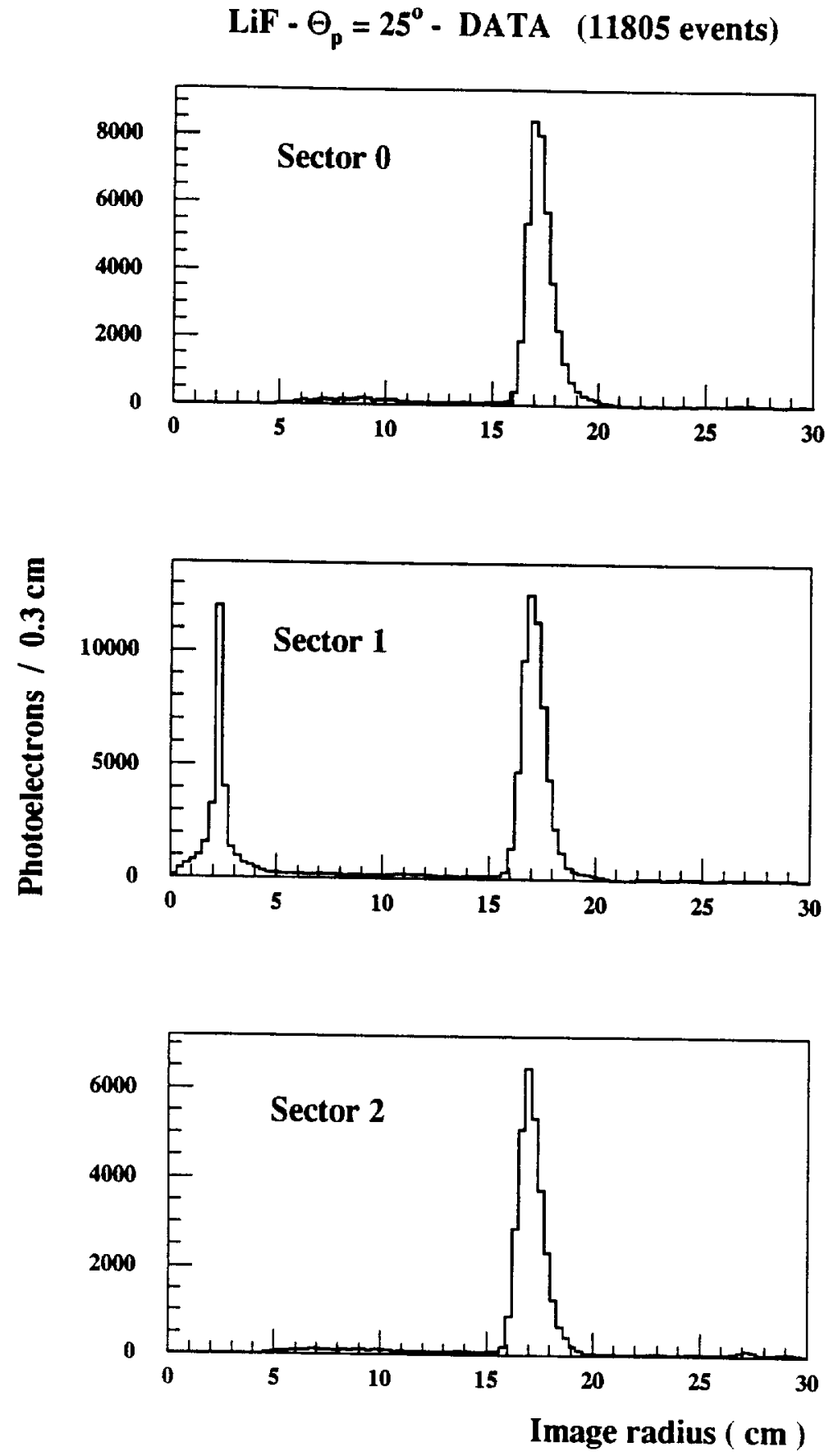

Figure 23. The single-photon reconstructed radius for each of the three sectors in the projection of Equations (9) for the full $\mathrm{LiF} 25^{\circ}$ data sample. 


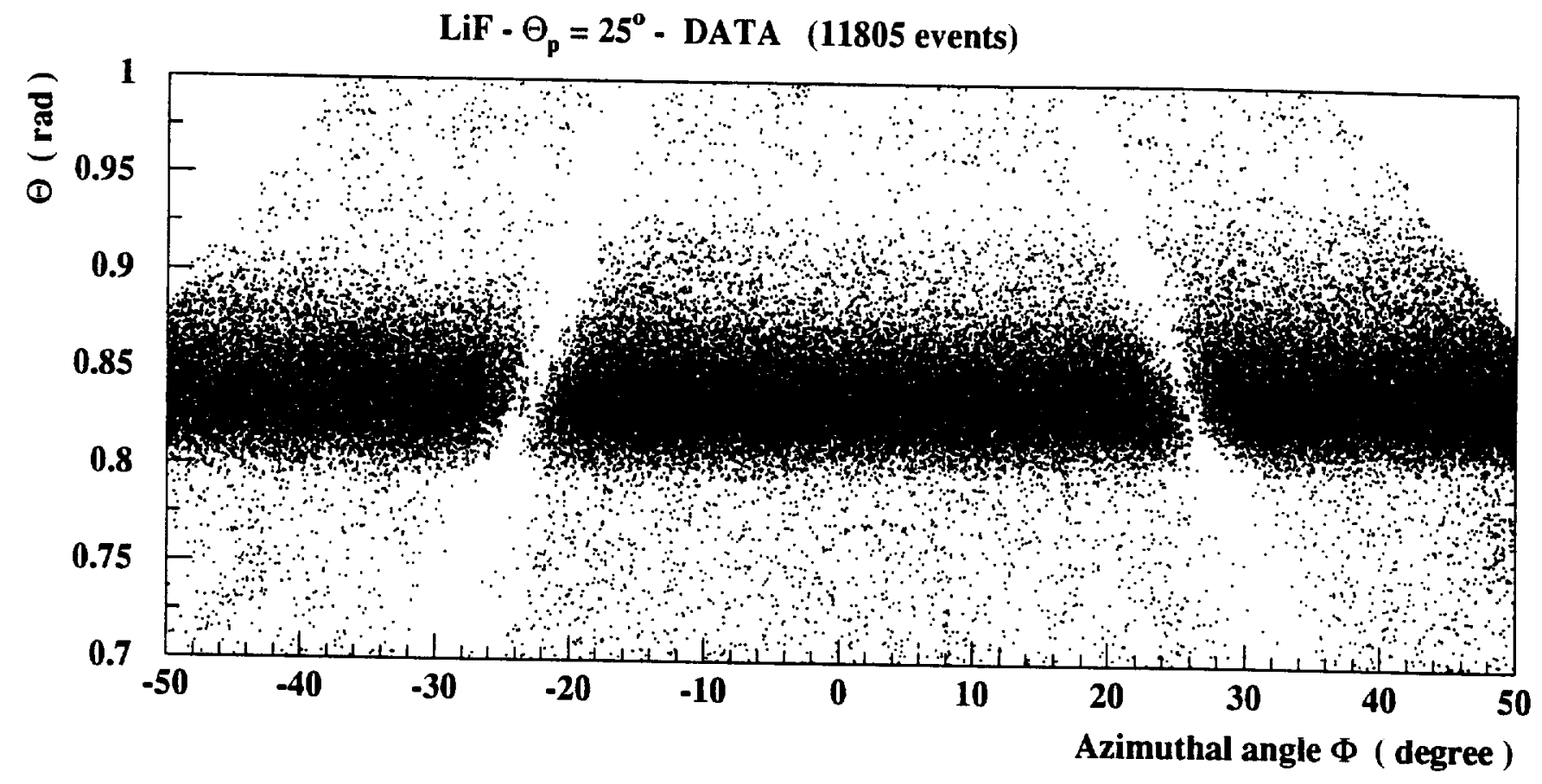

Figure 24. The scatter plot of the single-photon reconstructed Cherenkov polar angle $\theta$ vs. the reconstructed Cherenkov azimuthal angle $\phi$ for the full $\mathrm{LiF} 25^{\circ}$ data sample. The distribution is quite uniform in azimuth. 


\section{LiF $-\Theta_{p}=25^{\circ}$ - DATA (11805 events)}
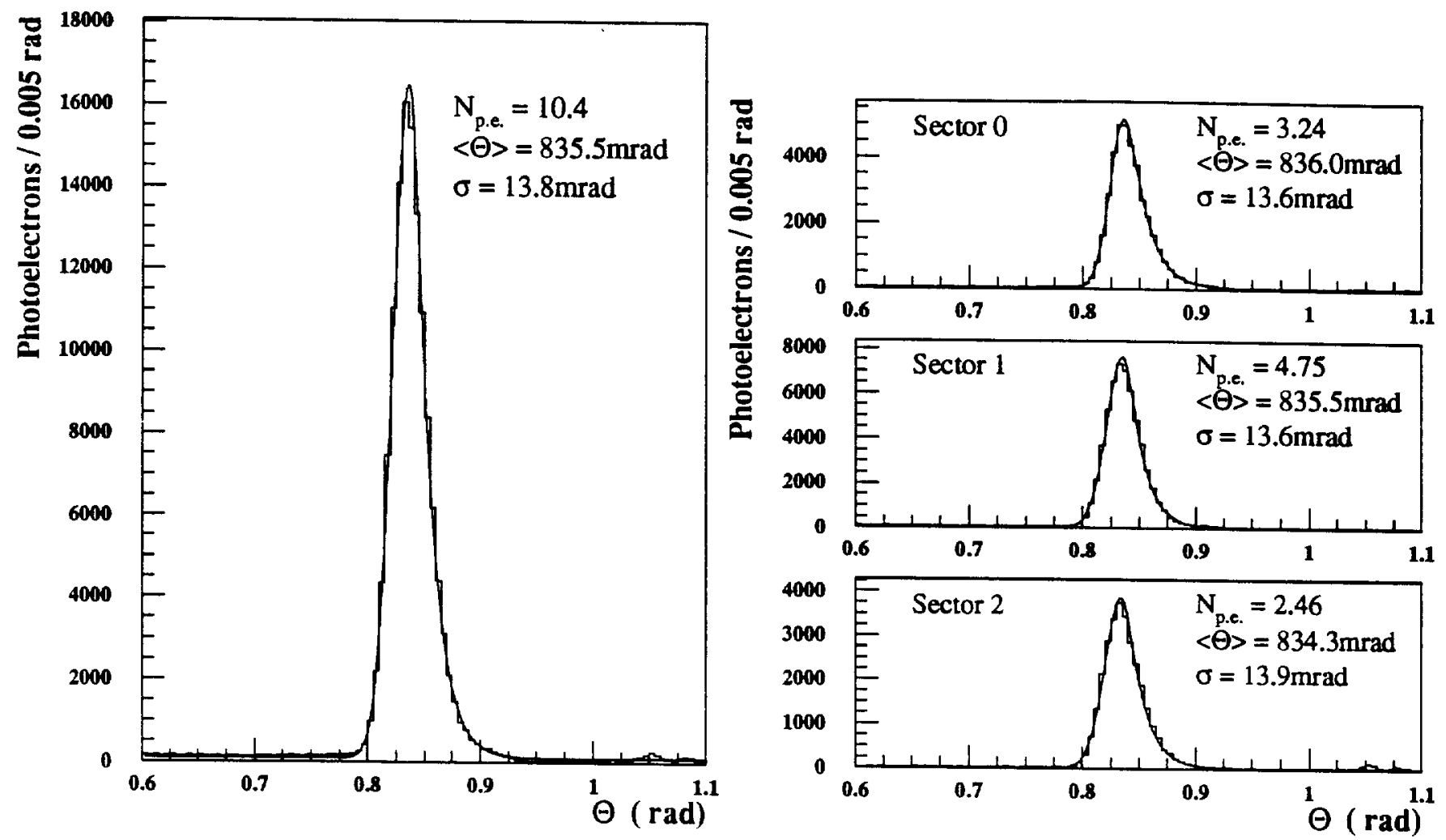

Figure 25. Reconstructed single-photon Cherenkov angle distributions for the full LiF $25^{\circ}$ data sample. The LHS shows the total distribution, the RHS that for each of the individual sectors. (See text for an explanation of the fit parameters.) 
LiF $-\Theta_{p}=25^{\circ}-$ DATA $(11805$ events $)$
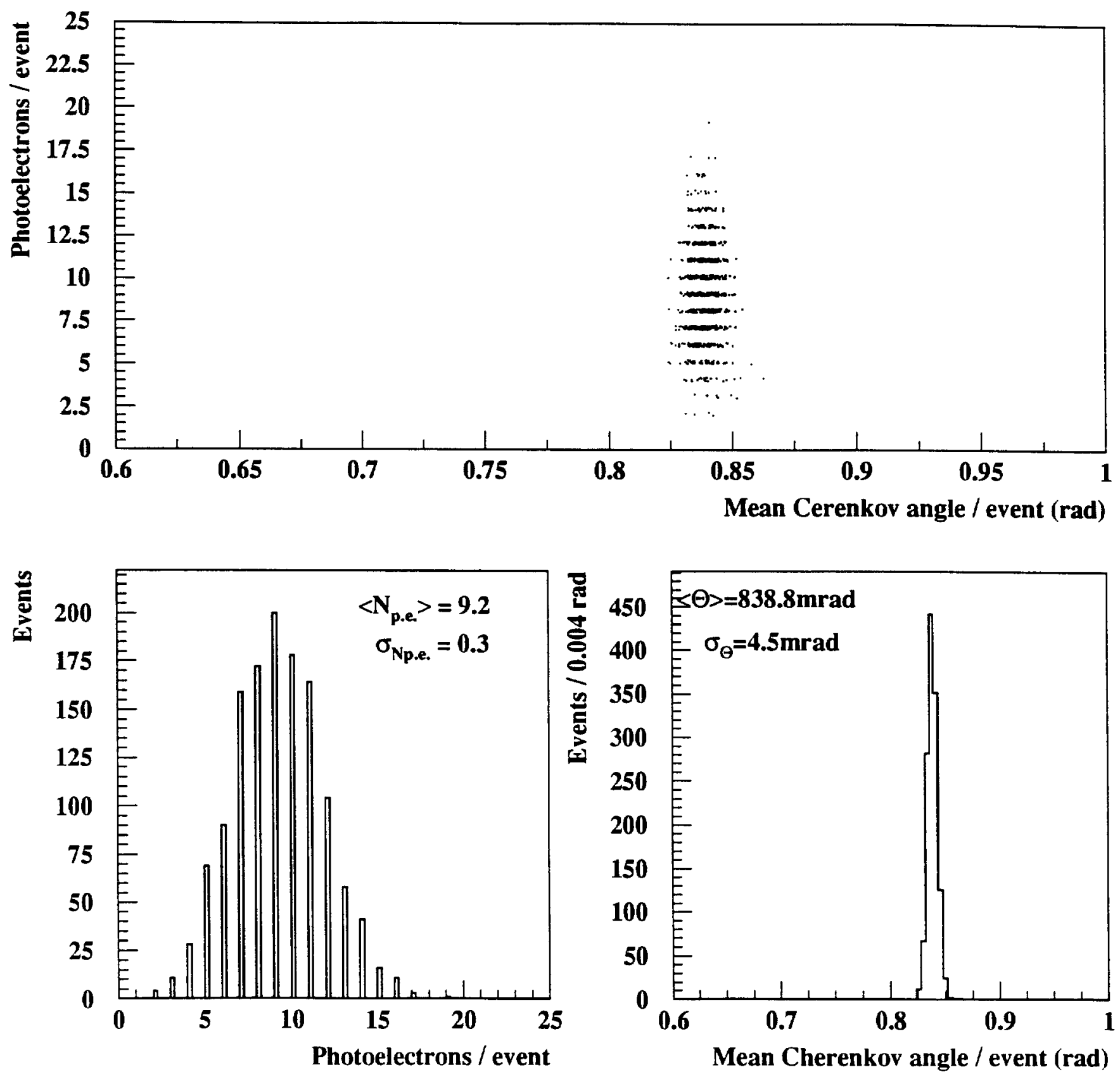

Figure 26. (a) A scatter plot of the (background subtracted) photoelectron yield $N_{\text {pe }}$ vs. the (mean) per event Cherenkov angle $\theta_{\text {evt }}$ for the full LiF $25^{\circ}$ data sample. (b) The photoelectron yield distribution. (c) The per event Cherenkov angle distribution. 
(a) $\mathrm{CaF}_{2}-\Theta_{\mathrm{p}}=30^{\circ}$ - DATA

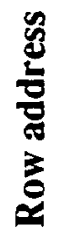

롤

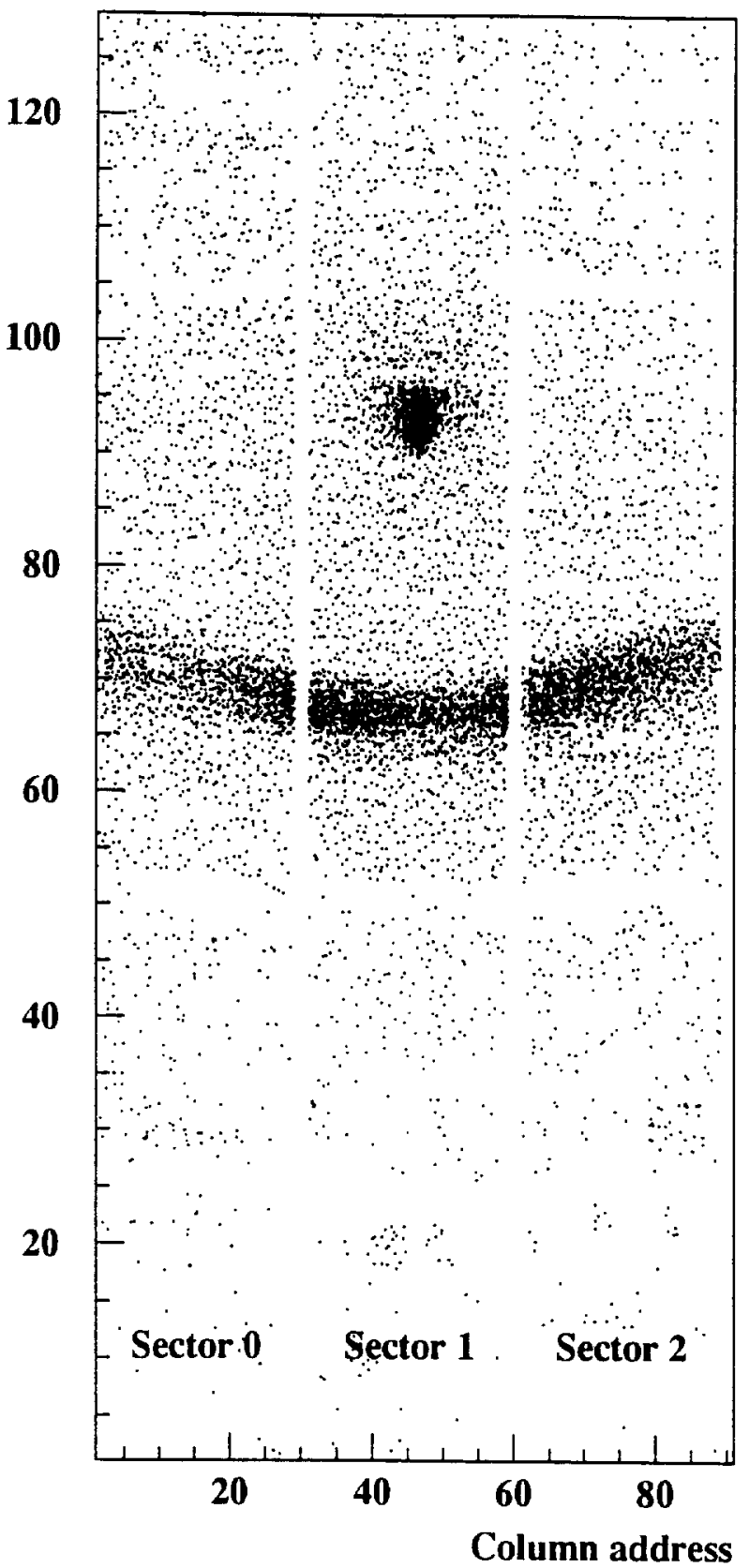

(b) No radiator $-\Theta_{p}=30^{\circ}$ - DATA

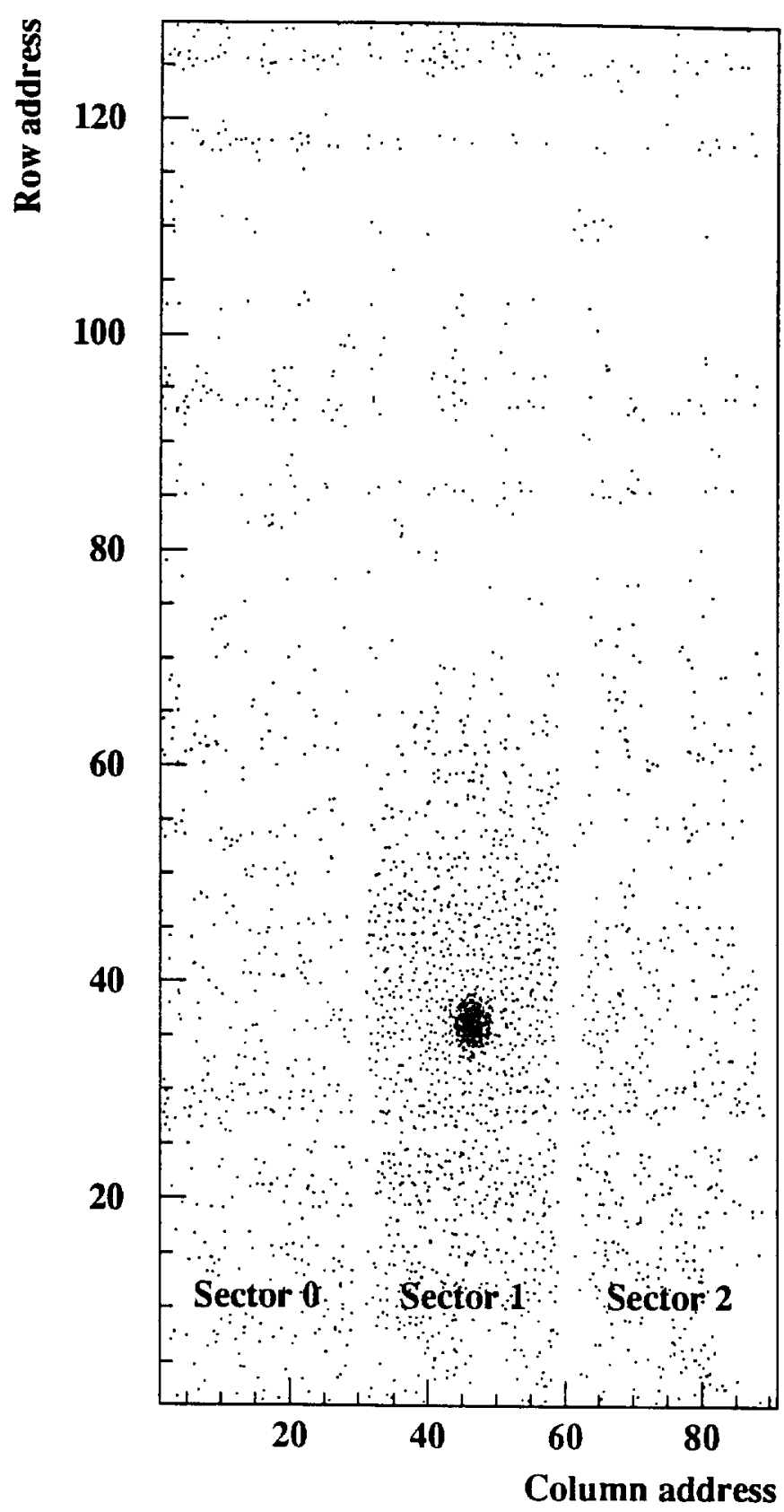

Figure 27. Raw events obtained in two runs of 5000 events at $\theta_{p}=30^{\circ}$, (a) with the beam centered on $\mathrm{CaF}_{2}$ radiator \#3, and (b) with no radiator. 
(a) $\mathrm{CaF}_{2}-\Theta_{\mathrm{p}}=30^{\circ}-$ DATA

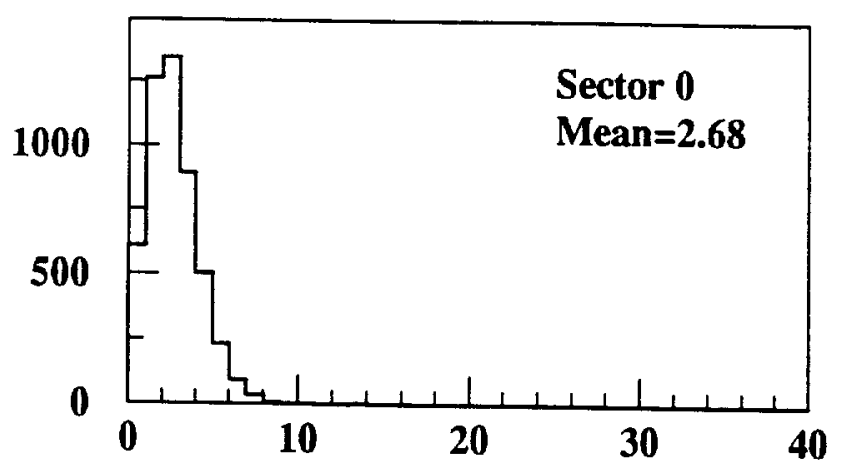

(b) No radiator $-\Theta_{p}=30^{\circ}$ - DATA

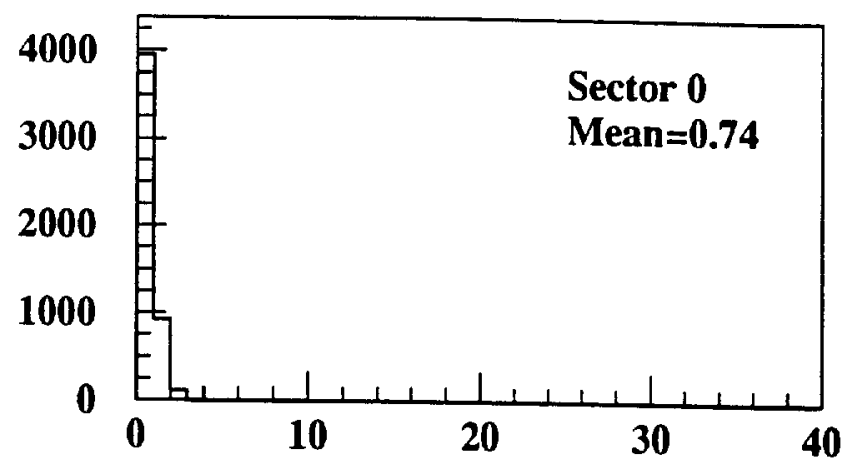

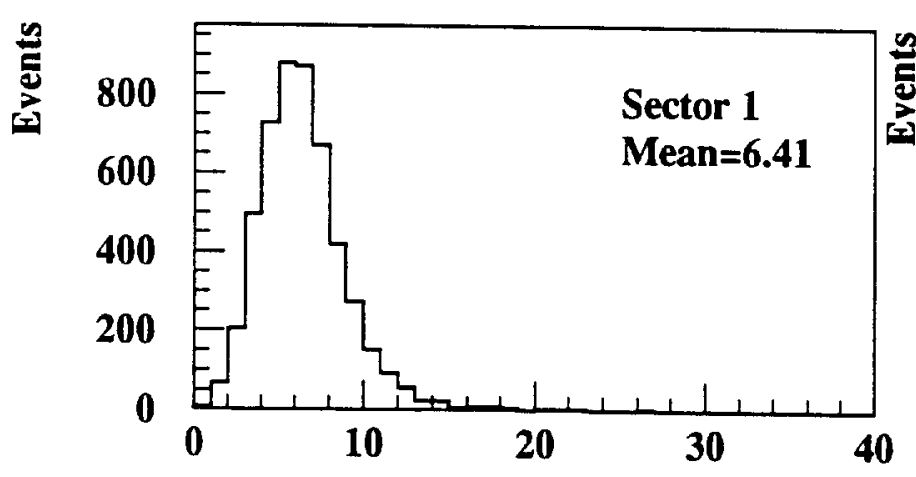
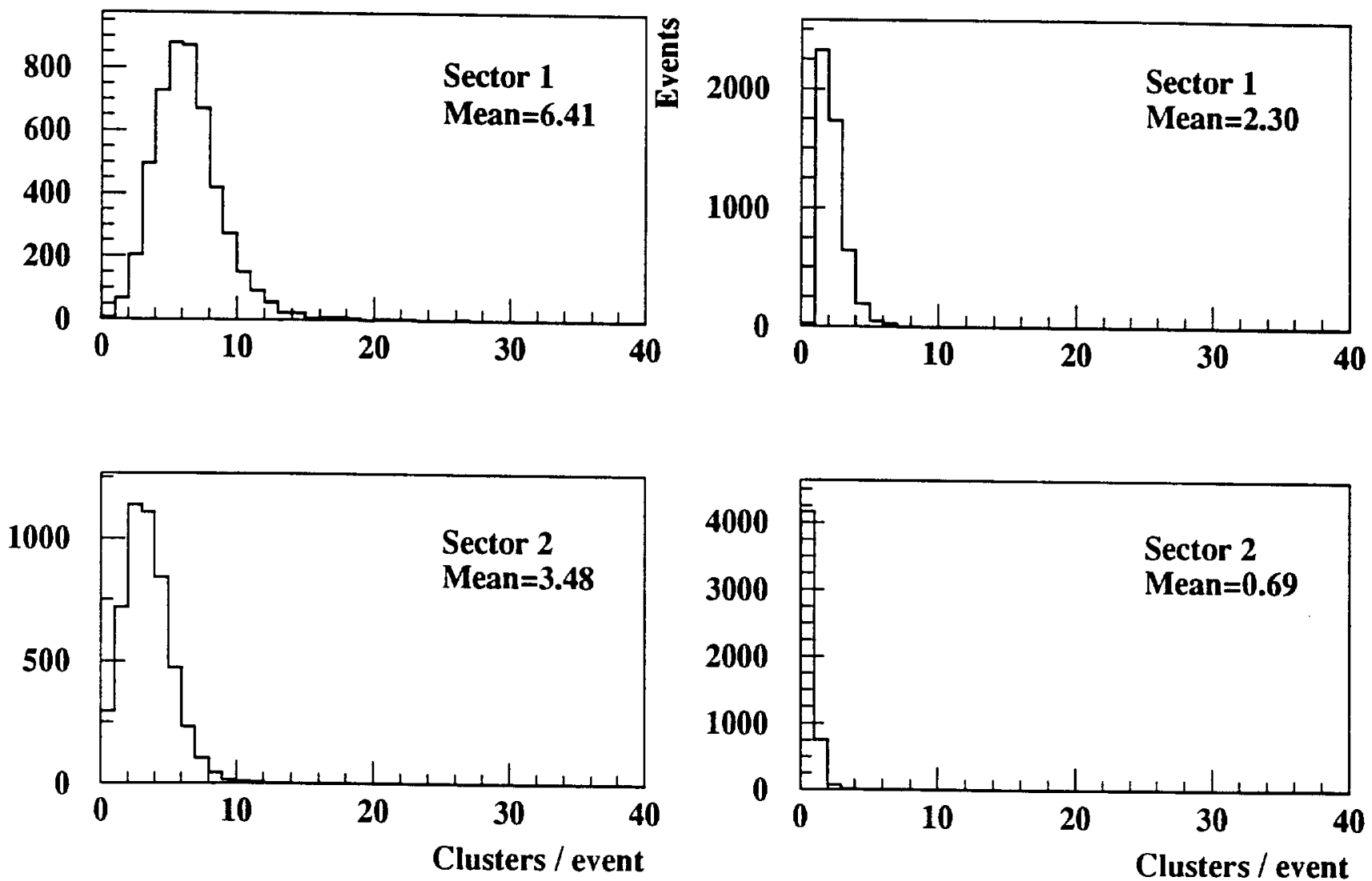

Figure 28. The distribution of the number of clusters per event per sector for the raw events of Figure 27, (a) with the beam centered on $\mathrm{CaF}_{2}$ radiator $\# 3$, and (b) with no radiator. 

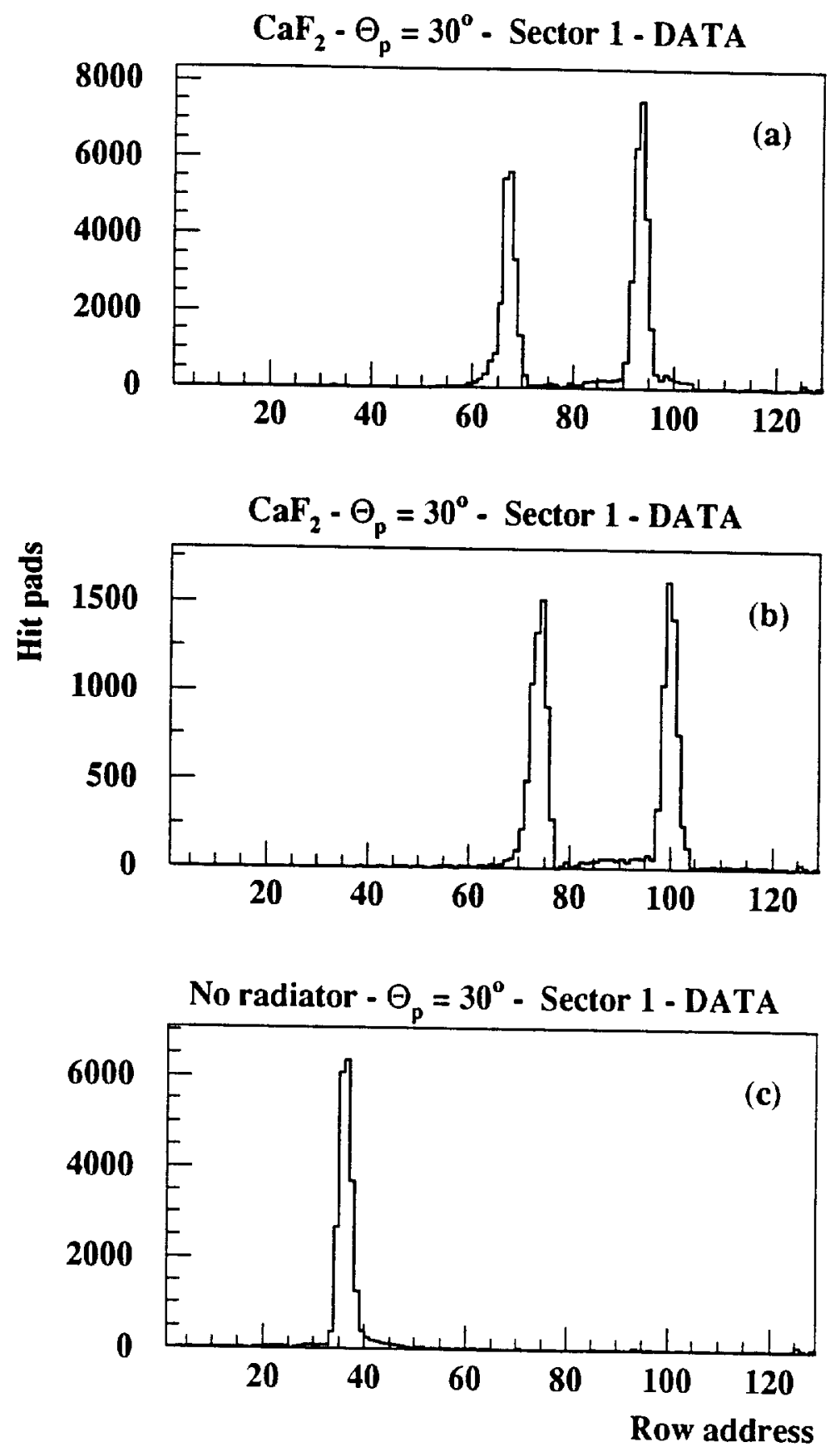

Figure 29. Projection of the raw events of Figure 27, showing a profile of the hit rows, (a) with the beam centered on $\mathrm{CaF}_{2}$ radiator \#3. (b) with the beam moved off-center of $\mathrm{CaF}_{2}$ radiator $\# 3$, (c) with no radiator. 
LiF $-\Theta_{p}=25^{\circ}-$ DATA $(11850$ events $)$

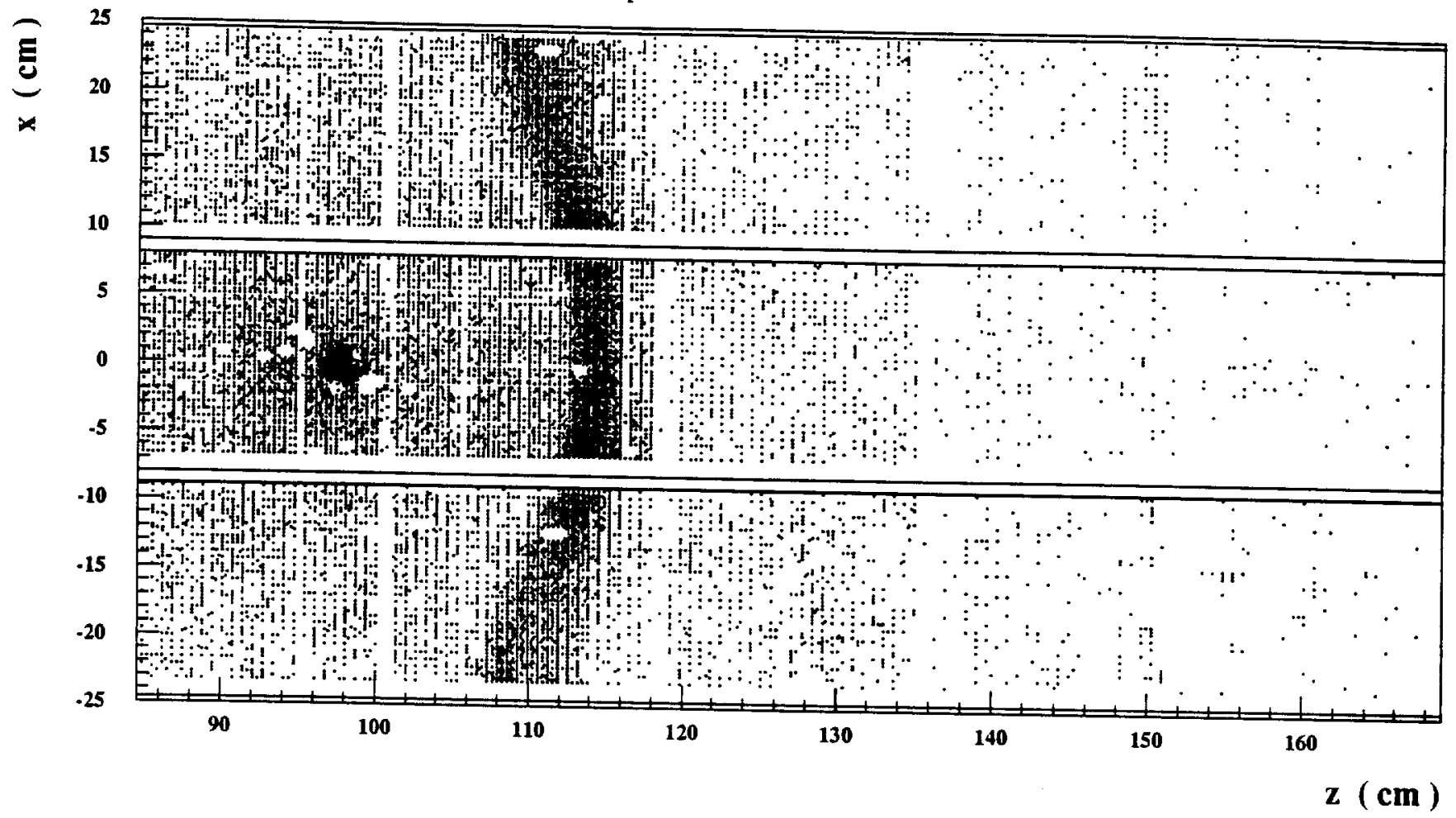

Figure 30. A scatter plot of reconstructed clusters projected into the photodetector plane of Sector 1. (See text for explanation of shadowing effects.) 


\section{$\mathrm{CaF}_{2}-\Theta_{\mathrm{p}}=30^{\circ} \cdot$ DATA (3822 events)}

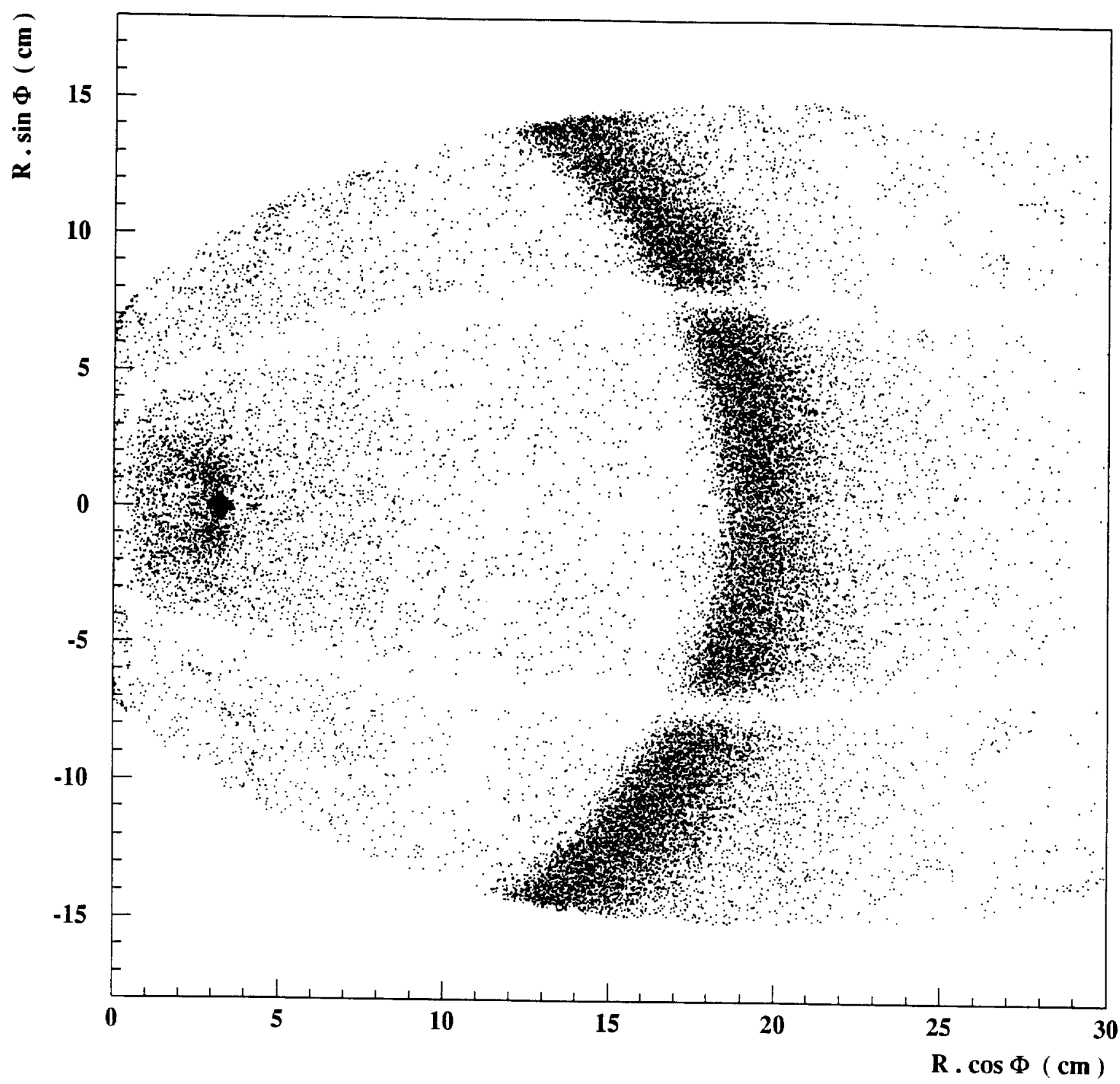

Figure 31. Cumulation of 3822 reconstructed images projected onto a plane perpendicular to the beam direction via the transformation of Equations (9). This is the full $\mathrm{CaF}_{2} \# 3 \theta_{p}=30^{\circ}$ data sample. 
(a) $\mathrm{CaF}_{2}-\Theta_{\mathrm{p}}=30^{\circ}-$ DATA (3822 events)
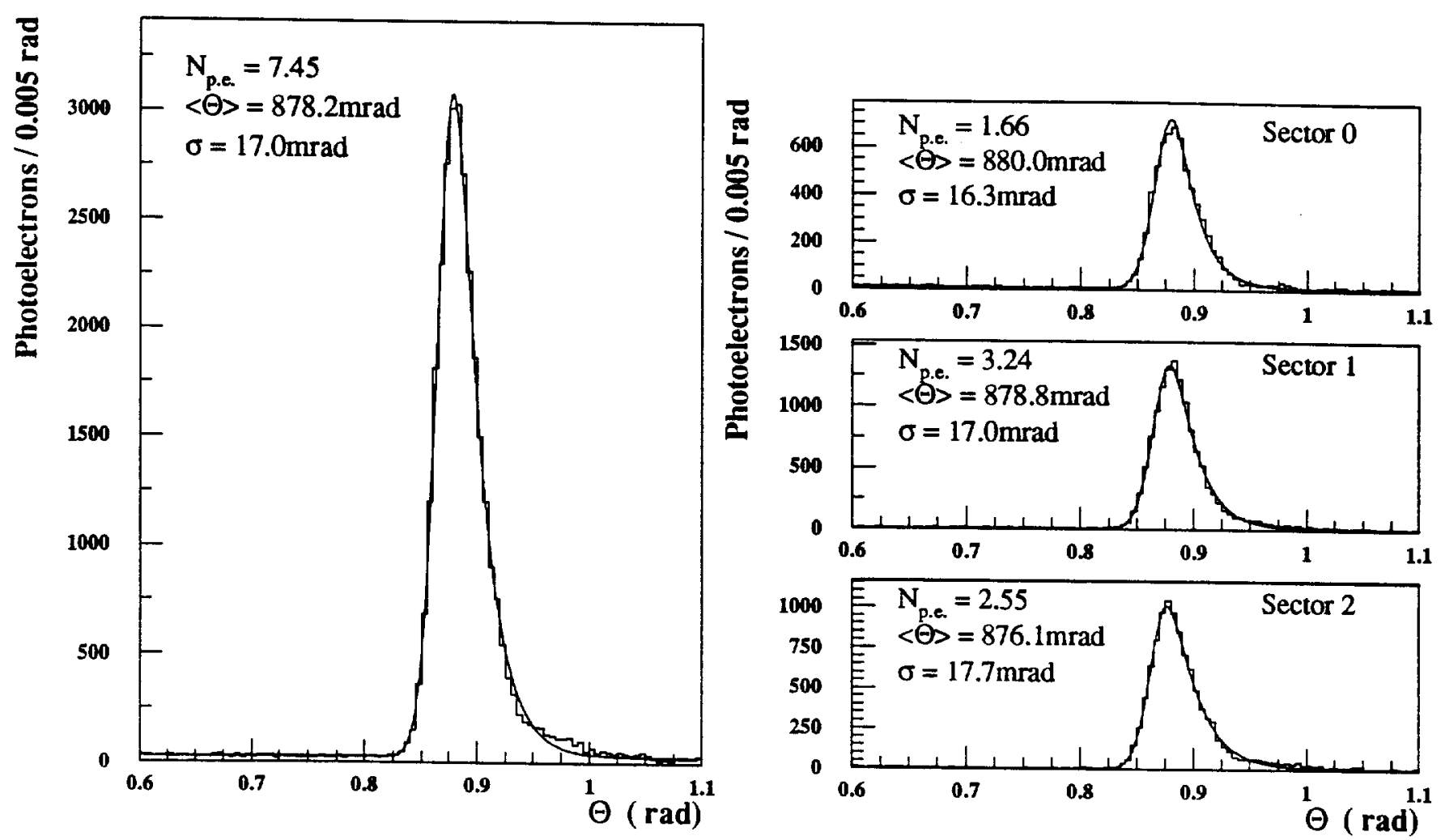

Figure 32. Reconstructed single-photon Cherenkov angle distributions for the full $\mathrm{CaF}_{2} \# 3$ $30^{\circ}$ data sample. The LHS shows the total distribution, the RHS that for each of the individual sectors. (See text for an explanation of the fit parameters.) 


$$
\mathrm{CaF}_{2}-\Theta_{\mathrm{p}}=30^{\circ}-\text { DATA }(3822 \text { events })
$$
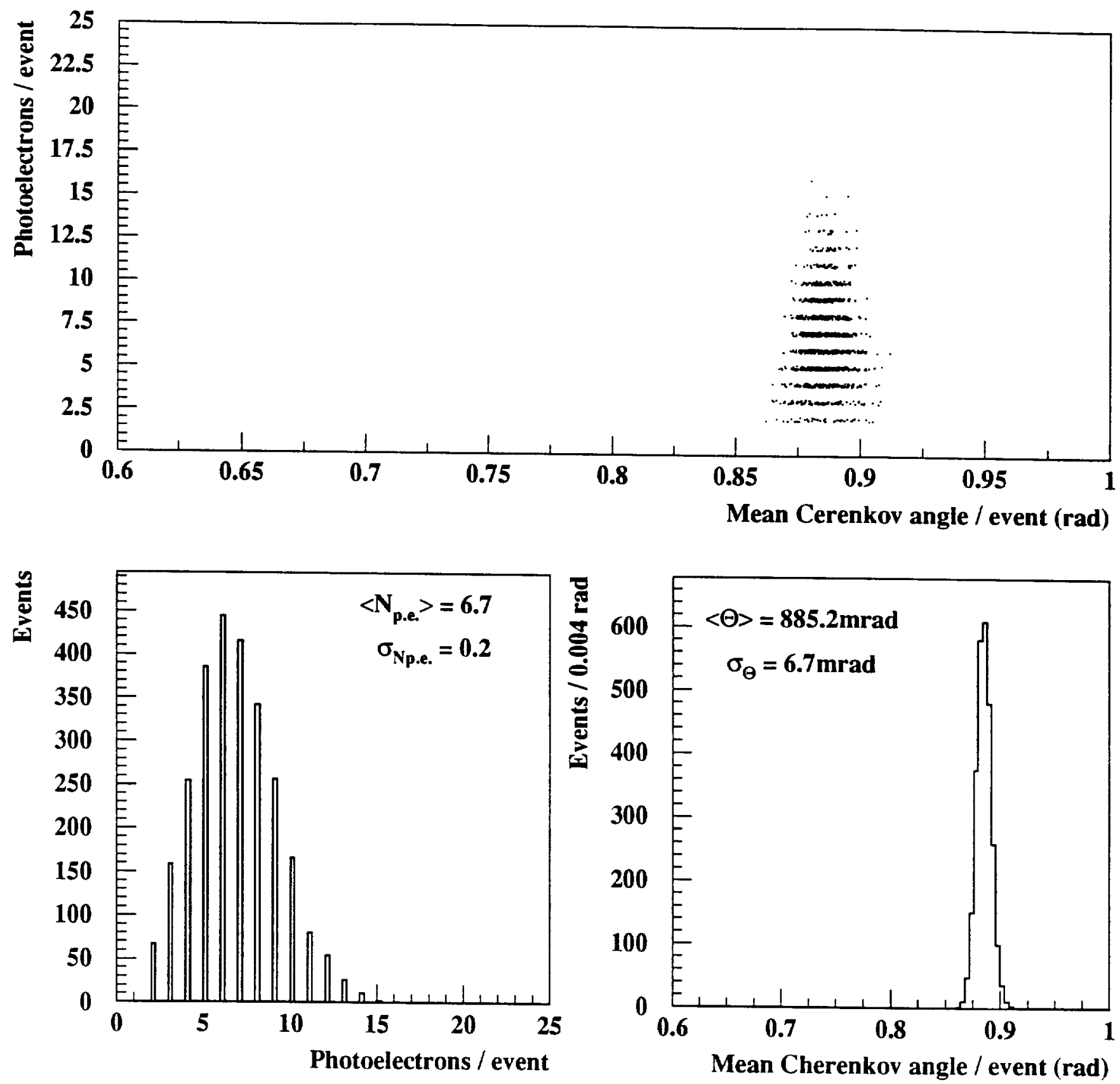

Figure 33. (a) A scatter plot of the (background subtracted) photoelectron yield $N_{\mathrm{pe}} v s$. the (mean) per event Cherenkov angle $\theta_{\text {evt }}$ for the full $\mathrm{CaF}_{2} \# 330^{\circ}$ data sample. (b) The photoelectron yield distribution. (c) The per event Cherenkov angle distribution. 


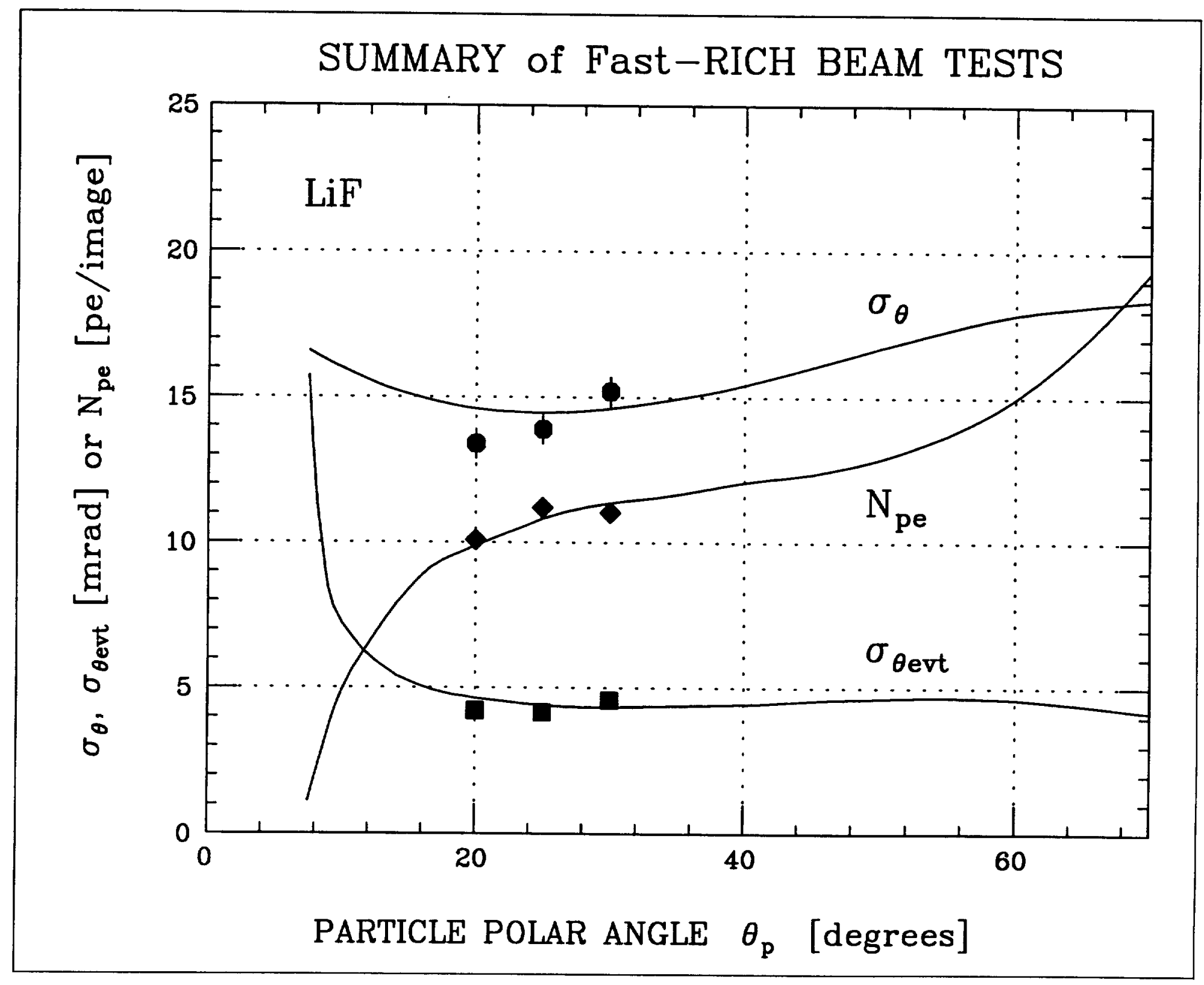

Figure 34. Summary of $\mathrm{LiF}$ results: A comparison of experimental values for $N_{\mathrm{pe}}^{\text {corr }}, \sigma_{\theta}$, and $\sigma_{\theta_{\text {evt }}}$ as a function of $\theta_{p}$ with an analytical calculation of the same. 


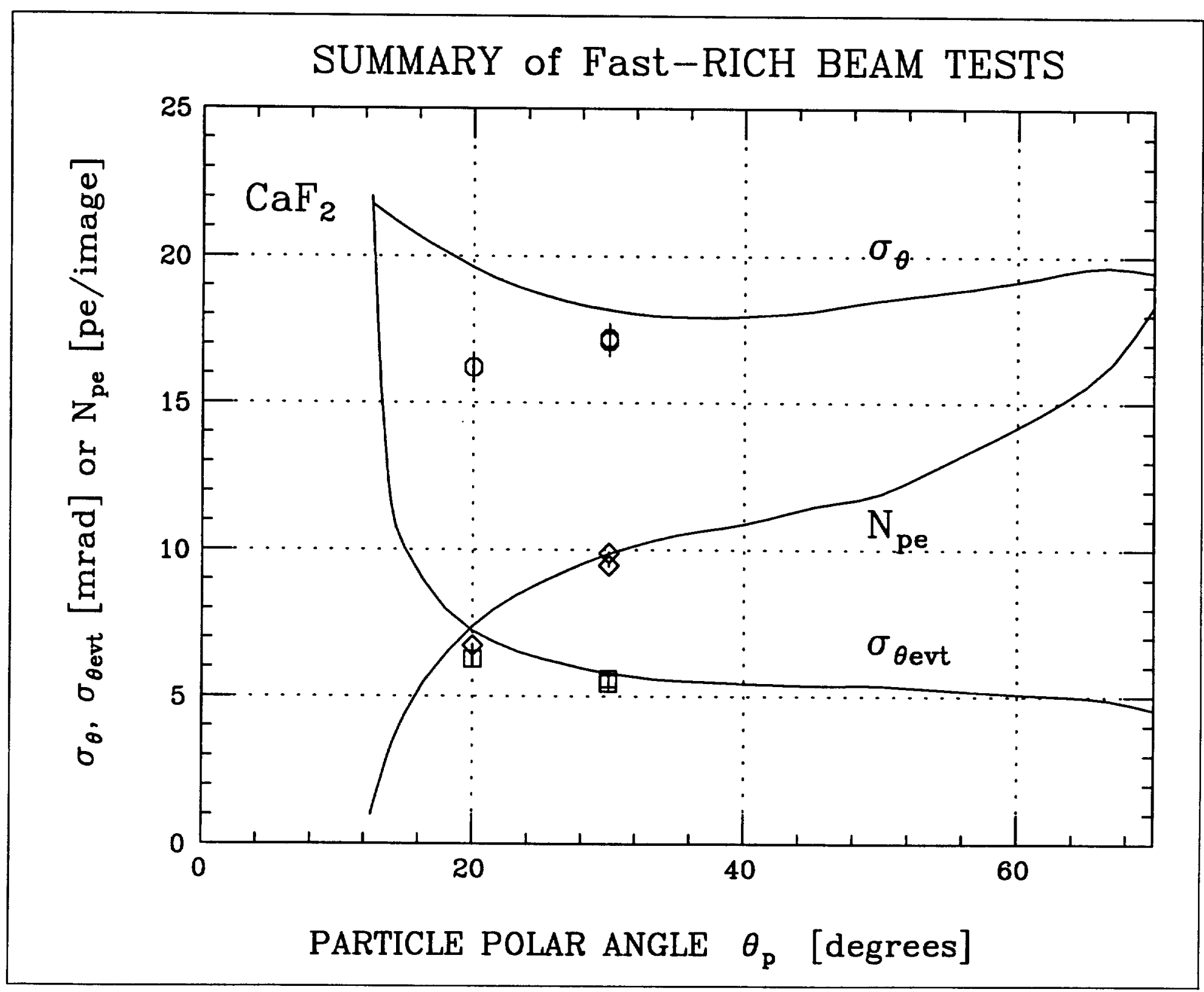

Figure 35. Summary of $\mathrm{CaF}_{2}$ results: A comparison of experimental values for $N_{\mathrm{pe}}^{\text {corr }}, \sigma_{\theta}$, and $\sigma_{\theta_{\text {evt }}}$ as a function of $\theta_{p}$ with an analytical calculation of the same. 

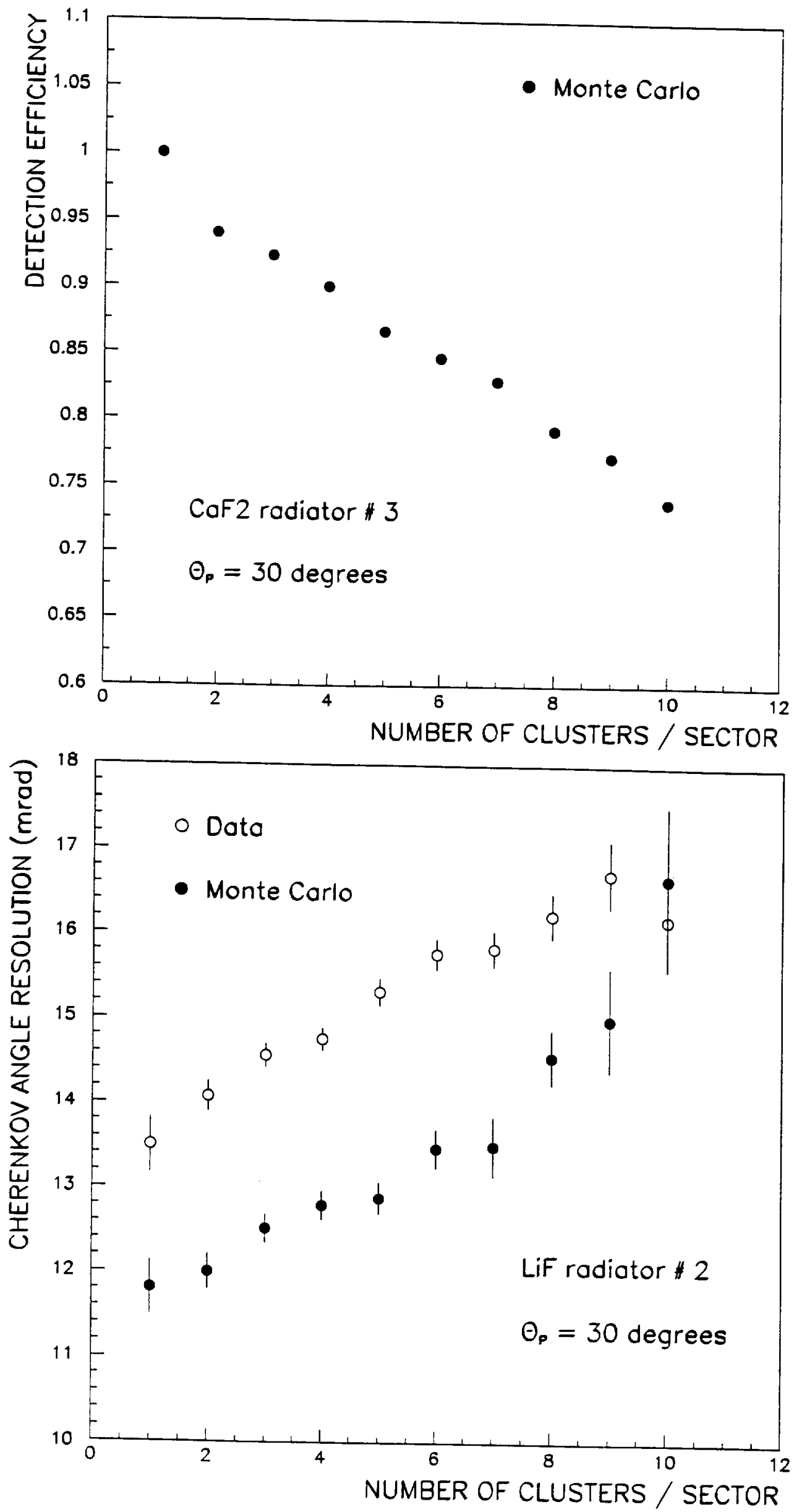

Figure 36. Effects of photon overlap. (a) Monte Carlo study of detection efficiency vs. number of clusters per sector. (b) Loss in Cherenkov angle resolution as a function of number of clusters per sector in both experimental and Monte Carlo data. 


\section{LiF $-\Theta_{p}=25^{\circ}-2 . G e V / c-$ DATA $(292$ events $)$}

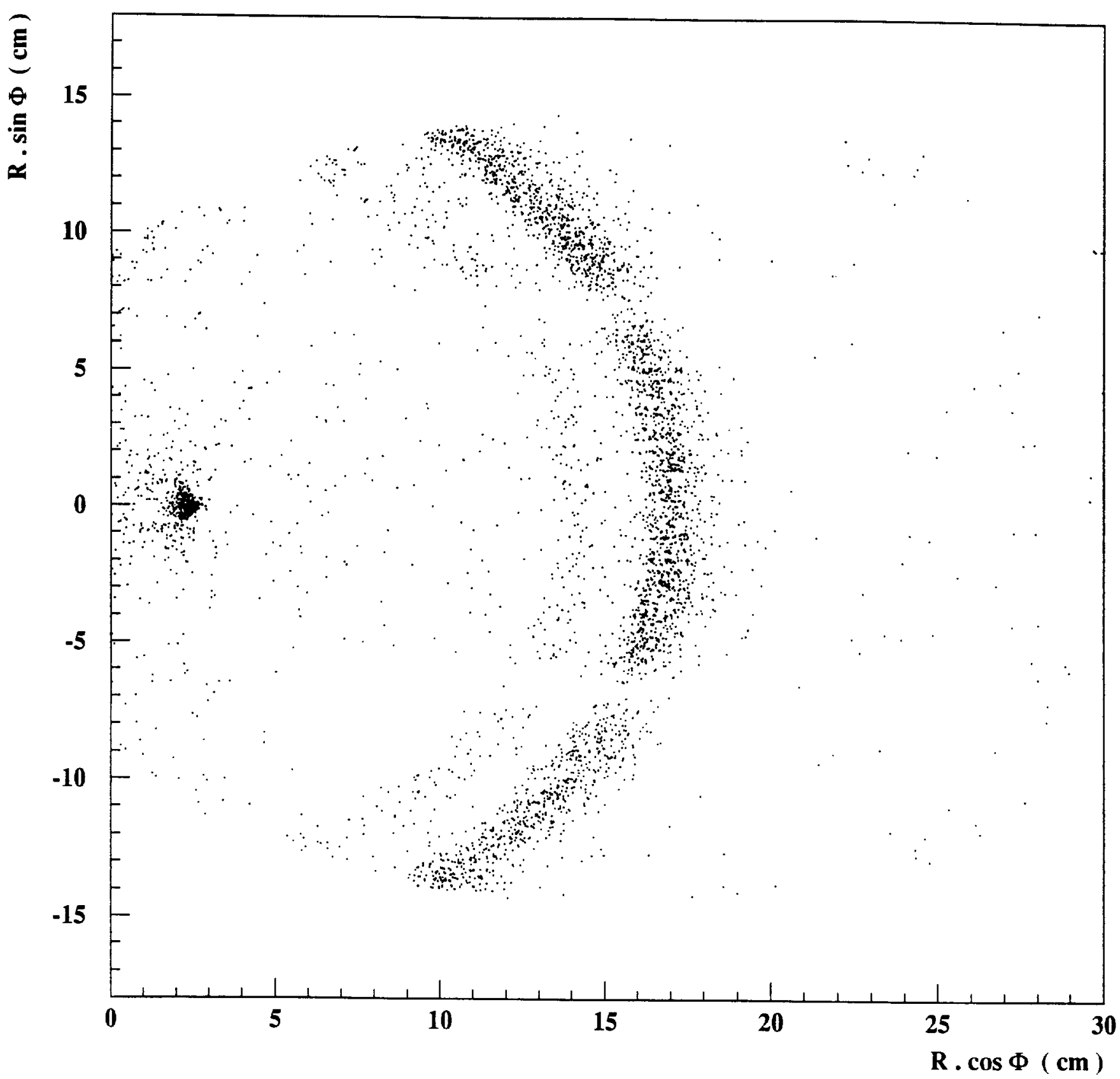

Figure 37. Illustration of the particle separation at $2 \mathrm{GeV} / c$. Cumulation of 292 reconstructed images projected onto a plane perpendicular to the beam direction via the transformation of Equations (9). Note the proton ring image concentric and interior to the pion ring image. This is the full $\mathrm{LiF} \theta_{p}=25^{\circ} 2 \mathrm{GeV} / c$ data sample. 


$$
\text { LiF }-\Theta_{p}=25^{\circ}-2 . G e V / c-\text { DATA }(292 \text { events })
$$
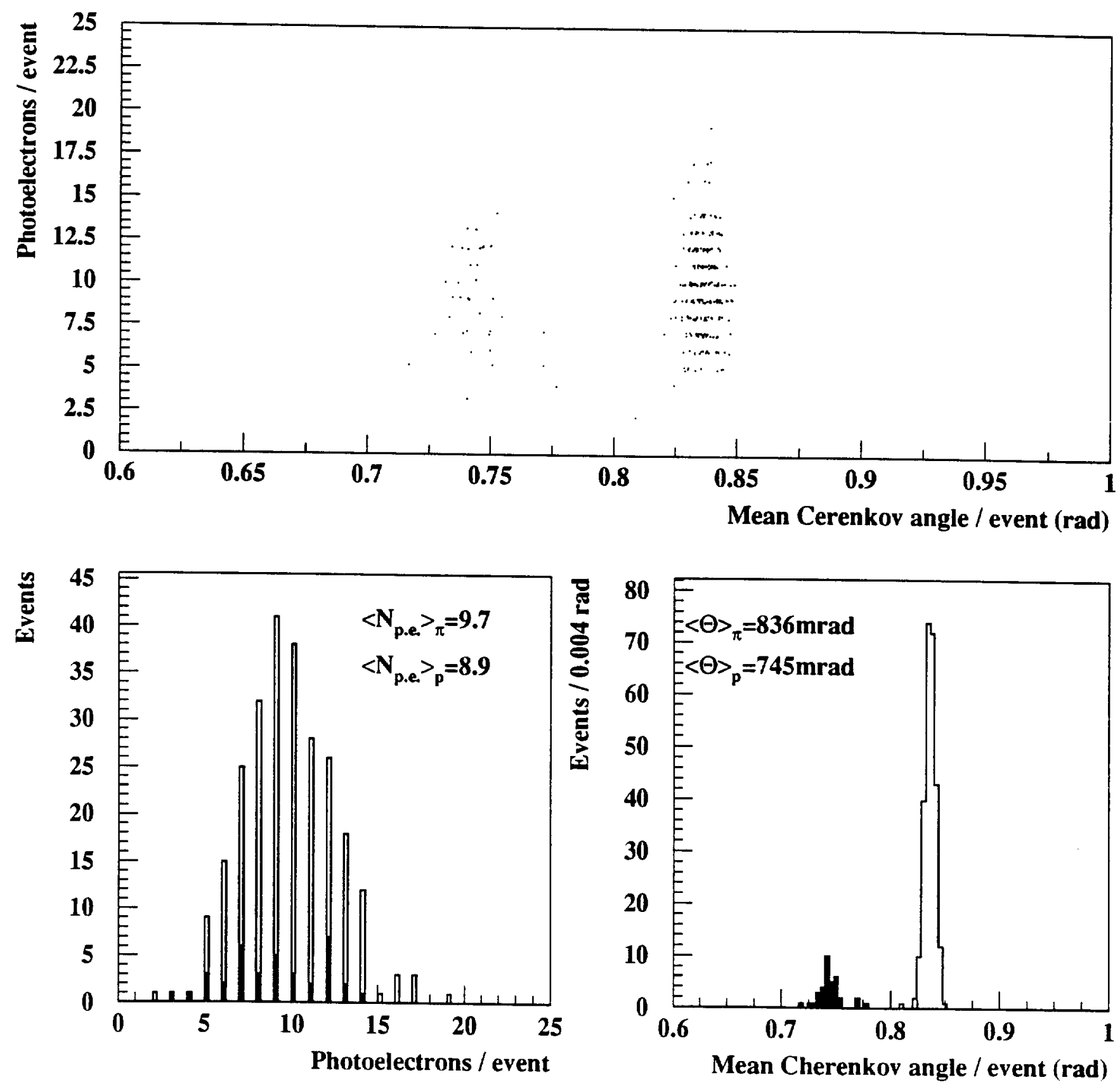

Figure 38. (a) A scatter plot of the photoelectron yield $N_{\text {pe }} v s$, the (mean) per event Cherenkov angle $\theta_{\text {evt }}$ for protons and pions in the full $\mathrm{LiF} 25^{\circ} 2 \mathrm{GeV} / c$ data sample. (See text for description of the particle determination.) (b) The photoelectron yield distribution. (c) The per event Cherenkov angle distribution, in which the separation of the two peaks is $\sim 20 \sigma$. 